\title{
Preventing the White Death: Tuberculosis Dispensaries
}

Egedesø, Peter Juul ; Hansen, Casper Worm; Jensen, Peter Sandholt

Published in:

The Economic Journal

DOI:

10.1093/ej/ueaa014

Publication date:

2020

Document version

Early version, also known as pre-print

Citation for published version (APA):

Egedesø, P. J., Hansen, C. W., \& Jensen, P. S. (2020). Preventing the White Death: Tuberculosis Dispensaries.

The Economic Journal, 130(629), 1288-1316. https://doi.org/10.1093/ej/ueaa014 


\title{
Preventing the White Death: Tuberculosis
}

\section{Dispensaries*}

\author{
Casper Worm Hansen
}

\author{
Peter Sandholt Jensen
}

\author{
Peter Egedes $\varnothing$ Madsen $^{\dagger}$
}

[Latest version: September 2017]

\begin{abstract}
Tuberculosis (TB) is a leading cause of death worldwide and, while treatable by antibiotics since the 1940s, drug resistant strains have emerged. This paper estimates the effects of the establishment of a pre-antibiotic era public health institution, known as a TB dispensary, designed to prevent the spread of the disease. Our annual differencein-differences estimation reveals that the rollout of the dispensaries across Danish cities led to a 19 percent decline in the TB mortality rate, but exhibits no significant impacts on other diseases in placebo regressions. We next take advantage of the dispensaries' explicit targeting on TB to set up a triple-differences model which exploits other diseases as controls and obtain a similar magnitude of the effect. Using monthly mortality data in a similar strategy leads to the same conclusion. In addition, we find small positive spillover effects of the dispensaries on productivity as measured by annual income per taxpayer at the city level, digitized from historical tax-assessment records. Overall, the evidence highlights the provision of personalized information on infectious diseases as a cost-effective cause of the historical mortality decline.
\end{abstract}

Keywords: Tuberculosis, mortality, disease prevention, information, rollout, productivity. JEL codes: D62, H23, I15, I18, N34.

\footnotetext{
*Acknowledgements: We would like to thank Jérôme Adda, Philipp Ager, Marcella Alsan, Martha Bailey, Sonia Bhalotra, Marianne Bitler, Hoyt Bleakley, Steve Broadberry, Aline Bütikofer, Karen Clay, Nicola Gennaioli, Claudia Goldin, Greg Clark, Janet Currie, Carl-Johan Dalgaard, Meltem Daysal, Dave Donaldson, James Fenske, Walker Hanlon, Ingrid Henriksen, Michael Haines, Erik Hornung, Aja Høy-Nielsen, Martin Karlsson, Adriana Lleras-Muney, Lars Lønstrup, Bentley MacLeod, Chris Meissner, Grant Miller, Myra Mohnen, Petra Moser, Therese Nilsson, Nathan Nunn, Per Petterson-Lidbom, Samuel H. Preston, Battista Severgnini, Paul Sharp, Marianne Simonsen, Mircea Trandafir, David Weil, Miriam Wüst, and seminar/workshop participants at the University of Copenhagen, the University of Southern Denmark, Copenhagen Business School, UC Davis, the University of Duisburg-Essen, the University of Giessen, the NBER SI DAE 2017, the Health and Gender: Global and Economic Perspectives Workshop at the University of Essex, the EEA 2016 meeting in Geneva, the AEA 2017 in Chicago, the H2D2 Research Day 2017 at the University of Michigan, the 7th European Meeting of the Urban Economic Association, and the Health and Labour Conference in Essen 2017 for useful discussions and suggestions. This paper was previously entitled "Information and Disease Prevention: Tuberculosis Dispensaries".

${ }^{\dagger}$ Contact: Casper Worm Hansen, University of Copenhagen, casper.worm.hansen@econ.ku.dk, Peter Sandholt Jensen, University of Southern Denmark, psj@sam.sdu.dk, Peter Egedesø Madsen, University of Southern Denmark, pe@sam.sdu.dk
} 


\section{Introduction}

An ounce of prevention is worth a pound of cure

- Benjamin Franklin

Living long and healthy lives is being increasingly recognized among economists as being paramount to human welfare and wellbeing (e.g., Nordhaus, 2003; Becker et al., 2005), and so understanding how to reduce mortality in a society is of first order importance for economists and other scholars (Cutler et al., 2006). This study investigates the effectiveness of information provision and isolation as public health policies to reduce inequalities in mortality across space and time and evaluate their direct, as well as their indirect, economic implications. In particular, we study a widespread (pre-antibiotic) public health institution, known as a tuberculosis (TB) dispensary, which had the primary purpose of preventing the spread of TB. We provide empirical tests on whether this type of institution was successful in reducing TB mortality and how it otherwise influenced the economy at large using Denmark as our laboratory. ${ }^{1}$

According to the World Health Organization (2015), TB is a major global health problem ranking alongside HIV as a leading cause of death. In 2014, there were 9.6 million new TB incidences, and even if the disease has been treatable by antibiotics since the 1940s, drug resistant strains now exist. ${ }^{2}$ It is therefore unsurprising that eliminating TB is part of the UN's third sustainable development goal. However, while TB is mainly confined to developing countries at present, European countries and the US also suffered from high rates of TB incidences and deaths before the advent of modern medicine. ${ }^{3}$ Moreover, there was considerable variation between these areas, as some countries were able to substantially reduce TB in the pre-antibiotic era. Daniels (1949, p.1066), for example, observes that the TB death rates fell in many European

\footnotetext{
${ }^{1}$ TB dispensaries were established in many other countries including Germany, France, United Kingdom, Sweden, Norway, and the United States during the same period. For example, we found that the number of dispensaries per capita in Massachusetts in 1920 was equal to dispensaries per capita in Denmark in 1939. In these years, both areas had 54 dispensaries and a population of around 3.8 million inhabitants according to the Annual Report of the Department of Public Health (1920) for Massachusetts, our own data, and population censuses.

${ }^{2}$ Tuberculosis was also known as the "the White Death" as used in the title. Other names include "the great white plague", "the robber of young", "the captain of all these men of Death", (Frith, 2014). "The White Death" is also the title of Dormandy's 1999 book on tuberculosis.

${ }^{3}$ The TB mortality rate in the United States fell from above 200 in 1900 to circa 60 per 100,000 in 1935 (Cutler and Meara, 2004).
} 
countries from above 200 per 100,000 in 1885 to below 100 in 1935 . He points out that "the most striking fall was in Denmark; the rate there was one of the highest recorded in Europe in 1885, with a mortality of nearly 300, and in 1935 it was below 50", which is the country where our quasi-experiment unfolds (i.e., our "laboratory"). Schelde Møller (1950) ascribed this decline to the policies pursued in Denmark, which were instigated by the National Association for the Fight Against Tuberculosis. Among other public health measures, the association established TB dispensaries locally, which were rolled out differentially across time and space. To our knowledge, this paper is the first to quantitatively evaluate the implications of establishing this particular TB institution. This allows us to consider the (cost) effectiveness of personalized information provision and isolation as public policy instruments to reduce mortality in a society. We also investigate their (indirect) economic effects. Therefore, our study relates more broadly to an ongoing discussion on health, productivity, and economic development (e.g., Acemoglu and Johnson, 2007; Bleakley, 2007).

The role of the dispensaries was to prevent the spread of the disease. Doctors would refer TB infected patients to the dispensaries, which would provide help, support, and examination of the infected individual and his or her family. They also attempted to ascertain how the patient contracted the disease and whether others had contracted the disease because of contact with the patient. A dispensary was little more than a room used for linen, towels, disinfectants, and spittoons (Schelde Møller, 1950). All doctors were able to refer patients to the dispensary, which was either led by a specialized doctor or nurse. ${ }^{4}$

Our main analysis estimates the impact of the TB dispensaries on TB mortality for Danish cities annually over the period 1890-1939. The first strategy employs difference-in-differences estimation, in which the impact of TB dispensaries is estimated using the rollout over time and across cities. The effect on TB mortality is negative and statistically significant, while performing a number of placebo tests reveals no impact on scarlet fever, pneumonia, accidents and suicides, diphtheria, cancer, polio, and influenza. Yet, the analysis indicates that overall and child mortality were negatively affected, albeit these effects are smaller than the direct effect on TB, as expected. Our second strategy follows a triple-differences setup, which exploits the

\footnotetext{
${ }^{4}$ See also Høy-Nielsen (2012) for an excellent description of the development of TB in the first half of the 20th century, based on the working of a TB dispensary in Ribe county.
} 
fact that the dispensaries were targeting only TB, and assigns the aforementioned diseases (i.e., causes of death) as controls. This experiment compares the development of TB mortality to the control diseases before and after the introduction of a dispensary in a given city. Reassuringly, both strategies result in similar estimates, suggesting that the opening of a dispensary lowers the TB mortality rate by around 19 percent.

For both strategies, we perform a number of robustness tests: for example, we have checked for differences in pretrends by estimating event studies that compute the average effect of having a dispensary in the 10 years before and after implementation. These estimates show that the impact of the dispensaries is systematically negative only after their introduction and that therea are no effects in the years before. These common pretreatment trends therefore provide indirect support for our main identifying assumption. Furthermore, random placebo tests that randomly reassign the intervention dates and then reestimate the models show that our baseline estimates are in the very left tale of the resulting distributions. Similarly, estimating models that allow effects from the opening of dispensaries in nearby cities suggests a very limited role for spillover effects. Along with a battery of other robustness checks, we also show that the results are not driven by, for example, pre-intervention population size, pre-intervention income, or pre-intervention inequality.

While our baseline estimates and robustness analyses are inferred using annual mortality data, we also provide evidence from monthly mortality data available at a higher level of geographical aggregation and from 1920 to 1939. As we have information on the exact date of establishment of the dispensaries, it is possible to fully exploit the high-frequency nature of these data. Following a strategy that resembles our triple-differences setup above, we find significant negative effects of the dispensaries on TB mortality and show that about 17-19 months after the introduction of a dispensary, TB mortality starts to decline, which resonates well with results from the annual event studies.

We also investigate the mechanisms by which the dispensaries could have reduced TB mortality, which, according to their policies, could happen in two ways (as also mentioned above). First, the TB doctor at the dispensary would inform the infected individual and his family about how to avoid spreading TB, and nurses would make home visits to assure that such guidelines were followed. We refer to this particular mechanism as personalized information 
provision. ${ }^{5}$ Second, the dispensaries would sometimes direct infected individuals to either TB hospitals or sanatoria, which effectively meant that they were isolated from the non-infected population (i.e., an isolation mechanism). ${ }^{6}$ In order to evaluate the relative importance of these two mechanisms, we collected an annual dataset with the number of patients in TB hospitals and sanatoria, and since we also know the historical location of these institutions, it is possible to test what happened to the number of institutionalized TB patients when the dispensaries were being rolled out in their local attachment areas. We find a negative effect on the number of sanatoria patients suggesting that personalized information provision is the most important one. ${ }^{7}$ The finding that personalized information is effective in changing behavior is also in line with evidence from health campaigns in contemporary developing countries (Thorton, 2008; Madajewicz et al., 2007).

Finally, after studying the effects on mortality, we estimate the economic impacts of the dispensaries. First, we have collected data on the cost of the intervention and find that the cost of saving a life year is around 68 dollars which, compared to previous studies that evaluate the cost effectiveness of other types of health interventions (e.g., Cutler and Miller, 2005; Bailey and Goodman-Bacon, 2015), makes the dispensaries extraordinarily cost effective. Second, we collected and digitized annual tax income data at the city level from historical tax-assessment records and find small positive spillover effects of the dispensaries on productivity (as measured by income per taxpayer). More concretely, we show that the introduction of a dispensary increased annual income per taxpayer growth by about 0.4 percentage points. This final finding supports recent research suggesting that eradicating TB is important for human capital accumulation and other economic outcomes (Hansen, 2013: Bütikofer and Salvanes, 2015). ${ }^{8}$ Also, circumstantial

\footnotetext{
${ }^{5}$ We note that the dispensaries also distributed some simple help remedies, such as glasses for sputum and clean bed linen, possibly making this type of information provision more effective.

${ }^{6}$ For example, the dispensaries directed 1,604 out of a total of 52,665 examined individuals (around three percent) to other TB institutions (e.g., TB hospitals and sanatoria). The percentage of individuals directed to other institutions decreased in the following years to around 0.45 percent in 1946 (Medical Reports, 1935-46).

${ }^{7}$ If the effect was mainly driven by isolation, one would expect to see an increase in the number of isolated patients; see also our discussion in the theoretical framework section. Also note that we demonstrate that the baseline findings are not confounded by the spread of these two other types of TB institutions, and we do no find any evidence suggesting that they by themselves reduced TB mortality in Denmark, which stands in contrast to Hollingsworth (2014), who shows that sanatoria in North Carolina reduced TB mortality among the white population, using an IV approach for identification. Nevertheless, Hollingsworth interprets his findings as suggesting that this was driven by information by the sanatoria.

${ }^{8}$ See Weil (2014) for a comprehensive overview of the literature that studies the effect of health improvements on productivity.
} 
evidence suggests that TB had important economic consequences. Fisher (1912), for example, provides a back-of-the-envelope calculation of the losses associated with TB in the United States and finds that they amount to roughly 1.2 billion US dollars in 1910 (about 3.5 percent of GNP). ${ }^{9}$

Using data for England and Wales, McKeown (1976) notes that 80 percent of the reduction in TB mortality happened before there was any effective medical treatment and generally argues that the declines in the mortality of diseases such as TB, dysentery, cholera, etc., were unrelated to any public health measures. This view has gained support by Fogel (1994; 1997); however, other prominent scholars, such as Preston (1975; ${ }^{10}$ 1996), Szreter (1988), and Cutler et al. (2006), highlight the important role of various public health interventions that were set in motion by the germ theory of disease in the $1880 \mathrm{~s} .{ }^{11}$ While recent research has shown that sanitation and clean-water supply were important drivers of the initial mortality decline in the United States (Cutler and Miller, 2005; Ferrie and Troesken; 2008; Clay, Troesken, and Haines, 2014; Alsan and Goldin; 2015), relatively little is known about how (and by how much) public health in the form of distribution of information about diseases has contributed to the historical mortality decline, which is one of the main contributions of our paper. ${ }^{12}$

Studying the impact of TB dispensaries in Denmark prior to the onset of modern medicine provides the advantage that any estimated effect cannot be confounded by provision of effective medicine. Moreover, as already indicated, TB dispensaries spread across European countries and US states after the first TB dispensary opened in Scotland in 1887, so in this way this type of institution was not unique to Denmark. However, focusing on the Danish case has the advantage that data are consistently available on a large number of diseases and the opening dates of the dispensaries, in a setting where national institutions cannot confound the results.

\footnotetext{
${ }^{9}$ Evidence from present-day Ecuador suggests that people contracting the drug-resistant strain are likely to earn less than 100 dollars per month due to disability (Rouzier et al., 2010).

${ }^{10}$ In Preston's seminal work of 1975, he argues that the upward movement of what has later been named the Preston curve (i.e,. the life expectancy-income relationship) is due to public health investments and health technological progress.

${ }^{11}$ See also Deaton's (2006) summary of the discussion between McKeown and Fogel on the one hand and Preston, Szreter, Cutler and Miller on the other.

${ }^{12}$ In a related study, Adda (2016) exploits contemporary high-frequency French data on the incidence of viruses and shows how they interact with human activities. For example, he demonstrates that school closures and the closure of public transportation networks reduce the spread of the diseases, but finds that these policies are not cost-effective, which is partly explained by the fact that their mortality is not that high in normal times.
} 
Apart from providing evidence on the link between information provision and mortality, there are at least two other reasons as to why studying the impact of TB dispensaries is interesting. First, they represent an example in which public health institutions may have mattered prior to the advent of modern medicine, contrary to what has been argued by McKeown (1976), Fogel (1994), and others. While we focus on the role of TB dispensaries, it is important to notice that we do not rule out that improved nutrition as emphasized by McKeown and Fogel may also have been important as well. Second, the dispensaries represent a clear illustration of a government response to the germ theory, which also has policy relevance today in a developingcountry context, as they suggest a way of combating an externality-producing infectious disease, especially because drug resistant TB now exists. ${ }^{13}$ Related to this, there is even substantial concern about drug resistant infectious diseases and their future consequences in developed nations as recently pointed out by O'Grada (2015; 2016).

The rest of the paper is organized as follows. Section 2 provides historical background on the TB dispensaries and discusses other aspects of the Danish policy. Section 3 outlines a theoretical framework. Section 4 describes the data along with the results of balancing tests. Section 5 explains the empirical strategies. Section 6 provides results, including robustness analyses. Section 7 reports results, using monthly-level mortality data. Section 8 provides evidence on the mechanism. Section 9 evaluates the economic consequences of the dispensaries. Finally, Section 10 concludes.

\section{Historical background}

This section explains the historical background and TB-targeted health policies in Denmark before the advent of antibiotics and vaccination. We first describe the medical situation prior to modern medicine and the historical development of TB mortality in Danish cities over time. We next describe the TB dispensaries in detail. Finally, we discuss other policy measures taken against TB.

\footnotetext{
${ }^{13}$ Building on Gersovitz and Hammer (2004, p.3), we note that an infection externality arises if, when choosing their level of preventive effort, people do not fully take into account the costs to others who will become infected as a consequence of their being infectious. The dispensaries would arguably reduce the private cost of taking proper preventive measures, as patients and relatives were instructed on how to avoid contagion so they could choose levels of preventive effor closer to the social optimum.
} 


\subsection{TB in Denmark, 1890-1950}

TB is caused by the bacteria of the Mycobacterium tuberculosis complex as discovered by Robert Koch in 1882. The most common type of TB occurs in the lungs - pulmonary TB-but TB can also affect other organs. Transmission of TB occurs by inhalation of infectious droplet nuclei containing viable bacilli, known as aerosol spread. Mycobacteria-laden droplet nuclei are formed when a patient with active pulmonary TB coughs, and they can remain suspended in the air for several hours. Sneezing or singing may also expel bacilli. After the initial infection of Mycobacterium tuberculosis, the individual either clears the infection, contains the infection without symptoms but with the bacilli remaining (i.e., latent TB infection), or develops active TB (Hemskerk et al., 2015). Individuals with a latent infection are at risk of developing TB from either endogenous reactivation or exogenous reinfection. Exogenous reinfections were particularly important in the past when TB was endemic in the population (Vynnycky and Fine, 1997). The most frequent symptom of TB is a non-remitting cough, which occurs in 95 percent of the cases. Many cases also include fever, nightsweat, and weight loss. TB is an ancient disease with the most conservative estimates being that it is 6,000 years old, and it has caused more deaths than any other disease during the last 200 years (Hemskerk et al., 2015).

In Denmark, TB mortality rates were high in the late 19th century and early 20th century. Importantly for our study, the first antibiotic effectively treating TB, streptomycin, was not invented until the 1940s. Moreover, vaccination against TB, the so-called Bacillus CalmetteGuerin (BCG) vaccine, was not applied systematically until the 1940s for the whole country, and only on the remote island of Bornholm from 1936-40 (Holm, 1946b). ${ }^{14}$ In fact, Holm mentions that BCG was carried out as an experiment of limited extent, as it was only given to a few newborn children. ${ }^{15}$ It should also be noted that the evidence on the effectiveness of the vaccine in stopping transmission is highly variable. According to Hemskerk et al. (2015), "BCG is the

\footnotetext{
${ }^{14}$ We perform robustness checks in which we exclude Bornholm as well as the period 1936-39 in our baseline sample.

${ }^{15}$ Holm (1946b) mentions that the first BCG strain was received by the Danish Serum Institute in 1927. The strain was used very cautiosly, which was good since it led to complication in vaccinated children. He notes that 16 children were vaccinated in 1927 in Copenhagen, seven in 1928, and 22 in 1929. As the strain used initially was "too potent" and in the 1930s a new strain was used. Holm also gives data from 1936-1941 for the central TB dispensary in Copenhagen. In 1936, the number of vaccinated children was 82 and in 1939 when we end our sample it had reached 492. The annual reports from the TB dispensaries only mention vaccination for Frederiksberg from 1936. As mentioned below, the program on the small island of Bornholm was much larger. As also noted below, we have checked whether these places drive our results, and they do not.
} 
most widely used vaccine in the world but measures of effectiveness have varied widely, between 0 and 80 percent. Studies have, however, consistently shown a protective effect against the most severe forms of childhood TB, including TB meningitis." This is corroborated by Nelson and Wells (2004), who note that meta-studies suggest that the protective effect is about 50 percent against pulmonary TB. Moreover, protection is stronger for TB meningitis, which children are more likely to develop (see below). ${ }^{16}$

Figure 1 shows the development of the TB mortality rate for the Danish city population from 1890 to $1950 .{ }^{17}$ There is a general decline in the mortality of the disease, as also observed by Daniels (1949). Yet, there is a spike around World War I as well as a plateau around World War II. We note that the general pattern of decline is not unique to TB but also holds for some other diseases such as pneumonia; see Online Appendix Figures A2-A9. This suggests that there are common causes behind the decline in mortality, such as improved nutrition, wars, and, for the late 1940s, modern medicine, which indicates the importance of controlling for time as well as other fixed effects in the empirical specifications.

\section{Figure 1: TB mortality in the Danish cities, 1890-1950}

Data also show that TB mortality and morbidity were declining simultaneously during this period. From 1921 to 1949, Schelde Møller (1950) provides data on mortality as well as morbidity for pulmonary TB for the whole of Denmark and, as revealed by Online Appendix Figure A12, pulmonary TB mortality and morbidity per 1,000 people followed similar downward trends with a correlation of 0.86 .

Online Appendix Figure A13 shows pulmonary TB mortality rates by gender for Copenhagen and the other Danish cities. Initially, males had higher death rates in Copenhagen though, these differences had almost disappeared by the 1930s. In the remaining Danish cities, differences by gender were less pronounced.

\footnotetext{
${ }^{16}$ Dormandy (1999, p. 347-349) reviews some of the early evidence on BCG. He notes that evidence from Gothenburg in Sweden indicated that BCG-vaccinated children "developed primary tuberculosis four to ten times less often and pulmonary tuberculosis tage three two to three times less often." (p. 348). In Britain, tests on 56,000 children starting in 1949 showed a pulmonary rate that was lower for the vaccinated children and no vaccinated child developed TB meningitis or military TB. Brimnes (2008) also reviews the evidence and covers the period after 1949. He cites studies that reported a protection rate of 36 percent in Georgia and Alabama in the 1950s. He also cite the Chingleput trial carried out from the late 1960s in India, which showed no protective effect.

${ }^{17}$ Online Appendix Figure A1 depicts a similar path for the pulmonary-TB mortality rate.
} 
Online Appendix Figure A14 shows the average age distribution of TB mortality for the five-year period 1921-25. ${ }^{18}$ It shows a wave-like pattern with high mortality among infants and young children and low mortality among the 5-15-years-old, after which mortality starts increasing in age to reach a peak in the early $20 \mathrm{~s}$. This pattern is consistent with the medical literature, which has found that very young children with an immature immune system are at high risk of developing TB when exposed to the infectious bacteria, whereas older children are the least likely, with the risk rising as they enter adulthood (Marais et al., 2005). Furthermore, children are more likely to develop TB outside the lungs, such as military TB and TB meningitis (Nelson and Wells, 2004; Smith et al., 1997).

\section{$2.2 \quad$ TB dispensaries}

The Danish National Association for the Fight Against Tuberculosis was established in 1901 and was originally focused on treatment, isolation, and patient care. However, in 1906 the secretary of the association went on a field trip to Germany to study the system of dispensaries that was being established there. Following this, the first TB dispensary was opened in Copenhagen in 1908 in a five-room apartment funded by a private donor for 10,000 Danish kroner, corresponding to about 645,000 Danish kroner in present value (around 95,000 US dollars). The dispensaries afterwards spread to other cities in Denmark, and they would often be led by specialized doctors, though some were led by nurses. Figure 2 shows the rollout of the TB dispensaries in a series of maps for different time periods. From 1908 to 1915, dispensaries were established in Copenhagen, Aarhus, and Odense (three of the largest cities), as well as in the smaller cities of Vejle and Slagelse. From 1916 to 1927, a few extra dispensaries were added but, as revealed by Figure 2, it was only from 1928 onwards that dispensaries started covering the whole country. The initial diffusion pattern is likely to reflect amendments to the TB law of 1905. The first amendment in 1912 states that private institutions focusing on the prevention of TB are eligible for state subsidies, and a second revision in 1919 implied that the dispensaries effectively became public institutions, although they remained organized by the National Association until 1928. Due to the work of medical director Johannes Frandsen the goal hereafter became to have a nation-wide network of dispensaries, led by specialized TB doctors, such that each county in Denmark would

\footnotetext{
${ }^{18}$ Data from earlier periods compiled by the National Association suggest a similar pattern.
} 
have one main dispensary with branches placed in other cities within the same county (Simonsen, 1947). This process was completed around 1944.

\section{Figure 2: Spread of TB dispensaries across Danish cities}

The dispensaries required only a room and spittoons, linen, towels, and disinfectants. Getting nurses with expertise in TB was initially a problem. This was solved by the National Association by offering specialized courses. From 1918, a cooperation between the association for nurses outside of Copenhagen and the National Association helped alleviate this problem. The lack of properly trained nurses has also been suggested as a fundamental reason for the slow initial spread of dispensaries (Permin, 1912; Schelde Møller, 1950).

Before the 1940s, the dispensaries had five different activities that helped prevent the spread of TB. First, local doctors would be responsible for new notifications to the dispensaries. Second, the dispensaries would perform consultations with patients and instruct them on how to avoid transmitting the disease further. Third, the dispensary would supervise a number of homes of TB patients. Fourth, nurses employed in the TB dispensaries would make home visits. Finally, they would direct patients to other TB institutions.

By 1927, 24 percent of the population had access to TB dispensaries, whereas by 1939, 67 percent had access (Medical Reports, 1927; 1939). In 1927, the dispensaries had 3,000 new patients and visited 15,000 homes (Medical Report, 1927). In 1939, 33,431 new individuals were referred to the dispensaries, 5,812 homes were supervised, 180,250 consultations were carried out, and nurses undertook 35,288 home visits (Medical Report, 1939). As mentioned, the dispensaries would also refer (some) patients to TB hospitals and sanatoria. For example, in 1935, the aggregate number was 969 patients, which then increased steadily in the following years; however, relative to the number of consultations the number was actually decreasing those years (Medical Reports, 1935-1946). For the 1940s, we also have some information on how much personnel the dispensaries were using: in 1943, for example, 89 nurses and 60 medical doctors were employed at 71 dispensaries. ${ }^{19}$

From the 1940s, vaccination became common, as mentioned above. The dispensaries performed vaccinations with statistics being reported systematically from 1943. Thus, to avoid

\footnotetext{
${ }^{19}$ As we note below, a method for diagnosing active TB was available from 1882 . Some dispensaries also had X-ray equipment, but this was not common in smaller areas prior to the 1940s (Holm, 1946a).
} 
confounding our results with any impact of the BCG vaccine, we use the period until 1939 as our baseline sample in the empirical analysis below, albeit results are robust to including the 1940s as well.

This subsection concludes by providing graphical evidence on the impact of the dispensaries. First, in Figure 3 we graph average TB mortality rates in the adopting cities 10 years before and after the introduction of a TB dispensary. While the TB mortality rate exhibits a downward trend, we see a clear discontinuity around the introduction of a dispensary. Second, in Figure 4 we graph the average number of monthly TB deaths across rural and urban areas up to 48 months before and after a change in the number of TB dispensaries. The figure shows that the number TB deaths fall after an additional dispensary was opened. We view these patterns as prima-facie evidence that the opening of the dispensaries mattered for the development of TB mortality.

Figure 3: Average TB rate before and after TB dispensary

\section{Figure 4: Average TB deaths 0 to 48 month before and after TB dispensary}

\subsection{Sanatoria and hospitals}

A number of TB sanatoria and TB hospitals were also founded during this time period. As with the dispensaries, the introduction of the sanatoria was inspired by German policies. Sanatoria were established around the country from the beginning of the 20th century and were often placed in the vicinity of a larger city, whereas the TB hospitals were placed in a city. The basic idea behind the sanatoria was that patients were given the best conditions for self-healing by getting fresh air and a balance between physical and mental rest on the one hand, and work therapy on the other hand (Schelde Møller, 1950). According to Rørdam (1911), sanatorium treatment was free for the poor and paid by local authorities and health insurance. The role of the sanatoria was to take care of the stronger patients, whereas weak patients were sent to TB hospitals. This practice lasted until the 1940s.

Porter (1999) argues that sanatoria only provided a holiday for their inmates and that there is little evidence that they mattered for the decline in TB mortality. The medical report for Denmark for 1903 contains circumstantial evidence that backs up this assessment with a 
statement from the chief medical officer of the medical district of Ringsted on Zealand, who had never observed any impact of a stay at a sanatorium. It is further observed in the same report that the sanatoria pick the stronger patients and leave the weaker patients to stay in hospitals in the cities. Others, such as Winge (1952), emphasize that sanatoria and TB hospitals provided both treatment and isolation, and the Bureau of Labor (1912) and Hollingsworth (2014) have a more positive assessment of the sanatoria. Our empirical analysis find little evidence that the TB hospitals and sanatoria reduced TB mortality by themselves and demonstrates that possible interactions between TB hospitals, sanatoria, and the dispensaries do not influence our baseline findings. In addition, we find that the dispensaries reduced the number of patients admitted to the sanatoria.

\subsection{Information campaigns across the country}

Active efforts to prevent the spread of TB by nationwide information campaigns were also in place. Signs reading "Do not spit on the pavement" were printed and distributed across cities, though they did not diffuse as much as the National Association had hoped for (Schelde Møller, 1950). From 1918, a poster with a similar message was sent to churches around the country. This type of information was distributed widely across the country, and we trust that time fixed effects will capture the campaigns in the empirical analysis. We also note that, in so far as the campaigns were effective, they would most likely also impact the spread of other airborne, infectious diseases such as pneumonia and scarlet fever as the mode of transmission is similar. As mentioned, we control for time fixed effects in all models, and we note that we can control for a tighter set of fixed effects when we use a triple-differences setup. Conceptually, one can think of these campaigns as general information provision, while the dispensaries provided personalized information.

\section{Theoretical framework}

This section outlines a Standard Inflammatory Response (SIR) model (Kermack and McKendrick, 1927) that has been modified to fit a pre-antibiotic population and TB environment as described 
in Section 2. ${ }^{20}$ We use this theoretical setup to clarify and illustrate how we think that the introduction of the dispensaries influenced the development of TB mortality. We use a discretetime version of the SIR model to facilitate the later comparison with our empirical annual panel-model counterpart. In this modified model, the total population $(P)$ consists of two subpopulations, which we are interested in following: the non-TB infected population $(S)$ and the TB infected population $(I)$. Only individuals with active TB are in $I$, which implies that latent TB individuals are grouped in $S$, as they are not contagious at this stage. Individuals in $S$ are at risk of being infected and developing active TB and thereby becoming part of $I{ }^{21}$ This risk is denoted by the infection rate, which is endogenous (see below). The other way for individuals to exit $S$ is by dying from other causes than TB. For simplicity, we assume that there is no co-mortality between TB and the other causes of death. Individuals in $I$ can either remain infected, die from TB, or recover, so that they again become part of the non-TB-infected population and, therefore, at risk of contracting TB later (since no short-run immunity against TB is granted after recovery), which is why we do not consider explicitly a specific group of recovered individuals as in the standard SIR model. The chance of recovery is related positively to income (see below). Finally, we assume that all individuals are born into the non-TB infected population. This simplification is not crucial in any way. Note that we do not incorporate any kind of immunization (treatment) instruments into the model, as our empirical analysis investigates the effect of the dispensaries on TB mortality in an environment without any "treatment" possibilities.

These assumptions imply that the non-TB-infected population develops according to:

$$
S_{t+1}=S_{t}+B_{t}-I_{t}^{N e w}-D_{t}^{\text {other }}+R_{t}
$$

where $B_{t}=b S_{t}$ is the total number of births ( $b$ is the exogenous birth rate in the non-infected population), $I_{t}^{N e w}=\lambda_{t}^{I} S_{t}$ denotes new TB infections $\left(\lambda_{t}^{I}\right.$ is the endogenous infection rate), $D_{t}^{\text {other }}=\lambda^{D} S_{t}$ is the total number of deaths due to other causes than TB, and $R_{t}=\lambda_{t}^{R} I_{t}$ is the total number of individuals recovering from a TB infection $\left(\lambda^{R}\right.$ is the recovery rate). The TB

\footnotetext{
${ }^{20} \mathrm{~A}$ variant of this model type is, e.g., also used in Adda (2016) to motivate his empirical analysis.

${ }^{21}$ Our model simplifies a little here, as in reality a TB infection might develop from latent to active without the individual being exposed to active TB (again). However, incorporating such a possibility does not change the theoretical predictions of the model (results are available upon request).
} 
infection rate is determined by:

$$
\lambda_{t}^{I}=\frac{\beta}{y_{t}^{\gamma}}\left(\frac{I_{t}}{S_{t}}\right)^{\alpha}
$$

where $y_{t}$ is income per capita and $0<\alpha, \gamma<1$. The first principal idea in equation (2) is that the number of infected individuals per non-infected population increases the risk that a random individual in $S$ comes into contact with a TB-infected individual. ${ }^{22}$ The second idea is that given the risk of getting into contact with an infected individual, the disease-specific infectivity constant $(\beta)$ discounted by the level of income $\left(y_{t}^{\gamma}\right)$ determines the probability of actually contracting TB. ${ }^{23}$ The infectivity constant is a function of:

$$
0 \leq \beta=(\sigma-\theta)(1-\delta) \leq 1
$$

where $\sigma$ denotes the contagiousness of TB, $\theta$ reflects the degree of awareness in terms of avoiding contracting/transmitting the TB infection $(\sigma \geq \theta)$, and $0 \leq \delta \leq 1$ is the share of infected individuals that are isolated. We think of $\theta$ and $\delta$ as the two parameters in the model possibly influenced by the dispensaries. As already indicated, the recovery rate is assumed to be a function of income:

$$
0 \leq \lambda_{t}^{R}=\bar{\lambda}^{R}-\frac{\Psi}{y_{t}} \leq 1
$$

where $0 \leq \bar{\lambda}^{R} \leq 1$ is the maximum recovery rate and $\Psi>0$ is a calibration parameter. Income per capita is assumed to grow at a constant rate:

$$
y_{t+1}=\left(1+g_{y}\right) y_{t},
$$

where $g_{y}$ is the growth rate. We see that according to equations (2) and (4), income growth feeds into the model through the infection rate as well as the recovery rate. The (active) TB

\footnotetext{
${ }^{22}$ This is a slight modification compared to the standard SIR model, which simply assumes $\lambda_{t}^{I}=\beta I_{t}$.

${ }^{23} \mathrm{We}$ discount the infectivity constant with income, as being well-nourished, for example, provides some immunity against contracting a TB infection. In addition, evidence suggests that reinfection (i.e., latent TB evolves into active TB) is less likely if people are well-nourished. As an alternative, we could have modeled the possibility of reinfection explicitly, such as:

$$
\lambda_{t}^{I}=\frac{1}{y_{t}^{\gamma}}\left(\beta\left(\frac{I_{t}}{S_{t}}\right)^{\alpha}+\lambda^{r e}\right),
$$

where $\lambda^{r e}$ is the reinfection rate, but this does not change the quantitative results of the model.
} 
infected population is given by:

$$
I_{t+1}=I_{t}+I_{t}^{N e w}-D_{t}^{T B}-R_{t},
$$

where $D_{t}^{T B}=\lambda^{T B} I_{t}$ is the total number of TB deaths and $\lambda^{T B}$ is the TB death rate among the TB infected population. Finally, we note that the TB mortality rate (out of the total population) is given by:

$$
M_{t}=\frac{D_{t}^{T B}}{P_{t}}=\lambda^{T B} \frac{I_{t}}{S_{t}+I_{t}} .
$$

Here we see that the two components of the TB mortality rate are $\lambda^{T B}$ and the incidence rate, $I_{t} /\left(S_{t}+I_{t}\right)$. Since we think of $\lambda^{T B}$ as being constant, any negative effect on the TB mortality rate due to the dispensaries (in this model), works through lowering the incidence rate.

Combining equations (1)-(7) yields a system of three first-order difference equations that characterizes how the populations and income evolve over time. This system is reported in equation (1) of the Online Appendix, along with a phase diagram sketching the solution (Online Appendix Figure A16). We also show that if $g_{y}=0, \alpha<1$, along with a couple of other plausible parameter restrictions, the model convergences to a constant ratio of TB-infected individuals to non-TB infected individuals (i.e., $\left.s \equiv I_{t} / S_{t}\right)$, implying a constant TB incidence rate, $s /(1+s)$, and TB mortality rate, $\lambda^{T B} s /(1+s)$. If $g_{y}>0$ these rates eventually converge toward zero.

This model is now used to quantitatively evaluate the adjustment process of TB mortality after the introduction of the dispensaries. Specifically, we simulate the development of the TB mortality rate after the introduction of the dispensaries (as measured by an increment increase in $\theta$ ) and compare it to a baseline simulation, which then corresponds to a counterfactual path without any changes in $\theta$. This type of comparison is equivalent to our (empirical) event studies, reported in section 6.3. The Online Appendix presents a detailed description of the parameter-value calibration of the model, but basically our strategy was to match the first moments in the 1908-data in population size, the TB mortality rate, and the crude death and birth rates. Figure 5 depicts the simulated differences between the path of the TB mortality rate with dispensaries and the counterfactual. Importantly, we see that only some four years later has the effect fully materialized. In this specific simulation, we also find that the TB mortality rate is 26 percent lower after five years, while the crude death rate has only decreased 
by around 1.5 percent, suggesting that we should find substantially smaller effects on the crude death rate than the $\mathrm{TB}$ mortality rate.

Finally, the model in general informs us that whereas a decrease in the TB mortality rate can be due to either an increase in the isolation factor or awareness, the isolation (population) rate, $q_{t} \equiv Q_{t} / P_{t}=\delta I_{t} /\left(S_{t}+I_{t}\right)$, would either increase if the effect is driven solely by isolation ${ }^{24}$ or decrease if the effect works through increased information. ${ }^{25}$ When we use these theoretical predictions, our empirical analysis later provides evidence that the decline in TB should more likely be interpreted along the lines of information provision or a combination of information and isolation, but not isolation on its own.

\section{Figure 5: Simulation of the TB death rate}

\section{Data and balancing tests}

This section presents an overview of the various data sets collected and digitized for the empirical analyses. Along with detailed accounts of the data sources, descriptions and definitions of all the variables are reported in Online Appendix Table A1.

Data on the timing of the TB dispensaries are collected and digitized from the annual medical reports "Medicinalberetning for Kongeriget Danmark" published by Sundhedsstyrelsen for the years $1908-1946{ }^{26}$ From 1908 to 1946, 98 dispensaries opened across the country, and during our baseline period of study (see below), 54 dispensaries were opened. We also obtain the date of commissioning of TB hospitals, sanatoria, and waterworks from the medical reports.

Out of the 54 dispensaries which opened between 1908 and 1939, 41 were located in cities so in order to evaluate their ability to reduce mortality ${ }^{27}$ we first digitized an annual city-level data set, containing information on eight different causes of death (see Section 5), the crude death rate, child mortality, the crude birth rate, and population size for 87 cities (i.e., all market towns

\footnotetext{
${ }^{24}$ This is under the assumption that the incidence elasticity with respect to $\delta$ is less than one. Nevertheless, it is clear that as $\delta \rightarrow 1$ this is not the case, since the incidence rate then becomes zero.

${ }^{25}$ For simplicity, we here assume that the number of isolated individuals is a fraction of all the infected individuals, and these two groups are similar in other observable aspects (e.g., the chance of recovery).

${ }^{26}$ Sundhedsstyrelsen, the National Health Department of Denmark, replaced det kgl. Sundhedskollegium in 1909, which had published the reports until then.

${ }^{27}$ In the empirical analysis, we are going to utilize the opening of the first dispensary in a given city. Because Copenhagen had three dispensaries, the analysis only exploits the opening of 38 dispensaries.
} 
in Denmark) over the period 1890-1950. The data are collected from the annual publications "Dødsårsagerne i Kongeriget Danmarks Byer" published by Sundhedsstyrelsen, which contains data from 1890 to 1919. By 1920, rural districts are added and the publication therefore changed its name to "Dødsårsagerne i Kongeriget Danmark", and from 1921 more cities are included due to the fact that some cities previously belonging to Germany became Danish after World War I. ${ }^{28}$ From 1901 these statistics become more detailed, and from this year onwards, we compiled a panel with eight causes of death (i.e., diseases), whereas from 1890 to 1900 only TB is available. In addition to the annual mortality data, we also collected and digitized, from the same publications, a second dataset with monthly mortality data on TB and 10 other diseases. These data are available at a relatively aggregate geographical level (i.e., urban and rural areas) from 1920 to 1939. Besides three dispensaries, for which we only have information on the year of opening, the medical reports provide the exact opening date for the dispensaries. The missing data on the exact opening dates, however, do not constitute a problem for our monthly analysis since the three dispensaries opened before 1918.

There are many reasons to believe that these historical mortality data are generally of high quality, and this seems to be particularly true for TB. Diagnosing TB became easier due to a number of innovations, for example, by "discovery of the acid-fast nature of the bacillus by Ehrlich in 1882, discovery of X-rays by Roentgen in 1895, development of the tuberculin skin test by Von Pirquet and Mantoux in 1907-1908" (CDC). ${ }^{29}$ The aforementioned discovery by Ehrlich allowed diagnosing active, rather than latent, TB by examining the sputum of a patient. ${ }^{30}$ According to Holm (1946a), diagnosing TB in Denmark included "a tuberculin test, roentgenography, and examination of sputum or gastric lavage for the presence of tubercle bacilli" and, therefore, the medical innovations were applied for diagnosis. In addition, disease registration on pre-printed forms had been in place in the cities since 1856 (Johansen, 2002), ${ }^{31}$ and on the quality of Danish (historical) medical statistics, Lindhardt (1938, p.28) observed that Danish and foreign investigators regard Denmark as being in the very front rank "as

\footnotetext{
${ }^{28}$ We refer to the "Dødsårsagerne i Kongeriget Danmarks byer" and the "Dødsårsagerne i Kongeriget Danmark" as the "Cause of Death Statistics".

${ }^{29}$ See: http: //www.cdc.gov/mmwr/preview/mmwrhtml/00000222.htm.

${ }^{30}$ Ehrlich famously self-diagnosed that he had TB in 1887; see Sakula (1982).

${ }^{31}$ Johansen (2002, p. 180) mentions that the TB mortality statistics for the 1890s are believed to underreport $\mathrm{TB}$, as it was sometimes mistaken for bronchitis. This is an additional reason for running some regressions from 1901 to 1939 .
} 
regards the conformity of the figures with the actual facts." She also highlighted that pulmonary tuberculosis is easy to diagnose at death and that Denmark had a large number of well-trained physicians. She examined death certificates for 1924-35 for patients who died of pulmonary TB and found that less than one percent did not have the cause of death affirmed by a physician. Her conclusion was that there is little risk for many deaths from pulmonary TB were being wrongly labeled. This evidence suggests that the quality of the vital statistics in Denmark is high by historical standards.

In the baseline, we stop the analysis in 1939, which is not crucial for the results, but we do so for three reasons. First, 1940-45 mark the years of World War II in Denmark. Second, the 1940s also mark the advent of modern medicine in which antibiotics for the treatment of TB became available. Third, and most importantly, the BCG vaccine became common over this period of time in Denmark, and it was often distributed via dispensaries, so in order not to capture a possible vaccination effect we stop the analysis in 1939. Descriptive statistics for the TB mortality rate and the seven other causes of death as well as death and fertility rates are given in Table 1, Online Appendix Table A2 describes changes in TB mortality over time, along with the number of dispensaries over time. The aggregate development of mortality, child mortality, live births, and the other causes of death are depicted in the Online Appendix Figures A2-A11.

Our third dataset, which we also collected and digitized, contains annual information on the number of patients admitted to all TB hospitals and sanatoria in Denmark (Medical Reports, various years). There were about 42 TB hospitals and 40 sanatoria (on and off) throughout this time period (1890-1939). Using their former addresses, we calculate the annual number of patients in each county, and our empirical analysis then estimates how these county numbers change when the dispensaries are being rolled out. While for the TB hospitals it is natural to use the number of dispensaries within the county as the attachment network, since patients could only be admitted to a TB hospital in their county of residence, this assignment rule is less straightforward for the sanatoria, as they could be used by patients (with a state subsidy) from across the country. Nevertheless, in order to make the results comparable, we use the same assignment rule for both institutions, which can also be supported by the presumption that people are more likely to opt for a sanatorium closer to their homes. 
Finally, we digitized income per taxpayer at the city level annually from 1904 to 1939, using historical tax-assessment records. These data are taken from "Statistiske Meddelelser", published by Statistics Denmark in the relevant years. These unique historical income data can be used to assess possible productivity spillover effects from the dispensaries, which, besides our cost-benefit analysis, allows for a broader evaluation of the total economic impacts. In addition, the robustness analysis for our mortality findings exploits initial variation in income (interacted with year fixed effects), along with within city income inequality, as controls. ${ }^{32}$

\section{Table 1: Summary statistics}

We conclude this section by commenting on a number of balancing tests reported in Online Appendix Table A3 in which we compare the population, population density, TB rate, death rate, child mortality rate, tax income per capita, and taxpayers per capita (as a proxy for labor force participation). Comparing the initial five-year mean of the variables between the TB dispensary adopters versus non-adopters, we observe no significant differences, although the TB rate in the adopting cities appear marginally larger and borderline significant at the 10 percent level, and the mean population size of the adopters seems larger, as it is driven by the few large cities in Denmark. ${ }^{33}$ Splitting the sample between the 18 pre-1930 adopters and the 20 post-1930 adopters, we only observe significant differences between the means of income per capita and taxpayers per capita, with pre-1930 adopters having higher means. In the robustness analysis, we show among other things, that our results are robust to controlling for the initial population, TB rate, initial income, and taxpayers per capita. Finally, Online Appendix Figure A17 depicts the relationship between the initial TB rate and the year of establishment of a TB dispensary for the unbalanced and balanced panels. The relationships are negative but far from being statistically significant.

\footnotetext{
${ }^{32}$ The within-city income inequality data are also obtained from Statistiske Meddelelser. Whereas the income data start annually in 1904, the first year with annual inequality data is 1918 .

${ }^{33}$ The initial five-year means are defined as the mean of the years 1890-94 for the population, population density, TB rate, and death rate; the mean of the years 1901-05 for the child mortality rate; and the mean of the years 1904-08 for the income per capita and taxpayers per capita. For cities included in the data set later, we use the mean of the first five years available.
} 


\section{$5 \quad$ Empirical strategies}

The first strategy in our empirical analysis is based on difference-in-differences estimation that compares the mortality rate of different diseases before and after the introduction of a TB dispensary in a given city:

$$
m_{c, t}^{d \in D}=\beta_{d} \text { Dispensary }_{c, t}+\alpha_{c}+\gamma_{t}+\mathbf{X}_{c, t}^{\prime} \theta+\varepsilon_{c, t}^{d}
$$

where $m_{c, t}^{d \in D}$ indicates the mortality rate in city $c$ in year $t$, Dispensary $_{c, t}$ is an indicator equal to one after the introduction of a TB dispensary in city $c$, the $\alpha_{c}$ 's are city fixed effects, the $\gamma_{t}$ 's are year fixed effects, and $\mathbf{X}_{c, t}$ is a vector of controls included in the robustness analysis. The error term, $\varepsilon_{c, t}^{d}$, is allowed to cluster at the city level and to be heteroscedastic. We estimate equation (8) for the following set of diseases $(d \in D)$ : TB, cancer, influenza, pneumonia, accidents and suicides (including homicides from 1931), scarlet fever, diphtheria, and polio. Since the principal purpose of a dispensary was to prevent the spread of $\mathrm{TB}$, we expect that $\hat{\beta}_{T B}<0$, while there should be no (or smaller) effects on the remaining diseases (see also the discussion below).

The second strategy exploits the explicit targeting of the dispensaries on preventing TB to estimate a triple-differences model, which takes on the following form:

$$
m_{d, c, t}=\beta \text { Dispensary }_{c, t} \times \text { Prevent }_{d}+\phi_{c, t}+\lambda_{d, t}+\mu_{d, c}+\varepsilon_{d, c, t},
$$

where the disease data have been stacked, such that $m_{d, c, t}$ is the mortality rate of disease $d$ in city $c$ in year $t$, Dispensary $_{c, t}$ is the same indicator as above but now interacted with Prevent ${ }_{d}$, indicating whether disease $d$ was prevented (i.e., treated) by the dispensary, which we assume was only the case for TB. The most important advantage of the triple-differences model is that it allows us to non-parametrically control for city-by-year fixed effects $\left(\phi_{c, t}\right)$, disease-by-year fixed effects $\left(\lambda_{d, t}\right)$, and disease-by-city fixed effects $\left(\mu_{d, c}\right) .{ }^{34}$ Thus, the quasi-experiment, which we now set up in the data, compares the development of TB mortality to non-treated diseases before and after the introduction of a dispensary in a given city, and we avoid comparing the

\footnotetext{
${ }^{34}$ We note that the interaction fixed effects $\left(\phi_{c, t}, \lambda_{d, t}, \mu_{d, c}\right)$ implicitly control for city fixed effects, year fixed effects, and disease fixed effects.
} 
development of TB mortality in larger to smaller cities, for example. The error term is given by $\varepsilon_{d, c, t}$ and is allowed to cluster at the city level and to be heteroscedastic.

We first note that the $\beta$ 's in equations (8) and (9) give the effects on the outcomes of being offered the possibility of treatment, which is known as an intention to treat (ITT) effect, circumventing the issue that at the individual level uptake is most likely endogenous. The main assumption of identifying the ITT effect in the difference-in-differences strategy is that cities getting a dispensary would have changed similarly to other cities, had it not been for the introduction of the dispensary, while the identifying assumption in the triple-differences strategy is that the diseases (in a given city) would have changed similarly in the absence of a dispensary. Indirect evidence supporting these assumptions is obtained by testing whether trends in our outcomes prior to the introduction of a dispensary are parallel, which is done by the following event studies:

$$
\begin{gathered}
m_{c, t}^{T B}=\sum_{j \in T} \beta_{j} \times \text { Dispensary }{ }_{c, t}^{\tau+j}+\alpha_{c}+\gamma_{t}+\varepsilon_{c, t}, \\
m_{c, t, d}=\sum_{j \in T} \beta_{j} \times \text { Dispensary }{ }_{c, t}^{\tau+j} \times \text { Prevent }_{d}+\phi_{c, t}+\lambda_{d, t}+\mu_{d, c}+\varepsilon_{c, t, d},
\end{gathered}
$$

where $T=\{-10, \ldots,-2,0, \ldots, 10\}$, and Dispensary $y_{c, t}^{\tau+j}$ is an indicator equal to one when $t=\tau+j$, where $\tau$ is the year in which a dispensary was established in city $c$, except for Dispensary $y_{c, t}^{\tau-10}$ and Dispensary $y_{c, t}^{\tau+10}$, which take on the value 1 given $t \leq \tau-10$ and $t \geq \tau+10$, respectively. The remaining variables are as defined above. The estimated coefficients $\hat{\beta}_{j}$ trace out the dynamic effects of the introduction of a TB dispensary relative to the omitted base year just before the intervention (i.e., $t=\tau-1$ ). For the common pretrend assumption to hold, we should find that $\forall j<0 \hat{\beta}_{j} \approx 0$. For example, systematically positively estimated coefficients prior to $t=\tau$ could indicate that a TB dispensary was introduced due to an unusually high level of TB mortality, while negative estimates could suggest that the rollout of the TB dispensaries is spuriously capturing a secular trend in TB mortality.

As the dispensaries were targeting TB, we expect there to be no (or much smaller) effects on the placebo diseases. However, this is ex-ante not necessarily the case due to competing risk and co-mortality. In the triple-differences strategy, competing risk would give cause a downward bias, whereas co-mortality would give rise to a bias in the opposite direction. The 
placebo-regression results (to be reported in the next section) suggest that there were no average effects on the placebo diseases considered here. Moreover, event studies do not reveal any evidence of co-mortality or competing risk even after 10 years (see Section 6.3).

Along with a range of other robustness checks, we also perform random-placebo tests that randomly reassign the intervention year and reestimate equations (8) and (9). By repeating this procedure 2,499 times, we obtain distributions of the estimated coefficients which can then be compared to the estimates found using the true intervention years.

\section{Results}

We begin the analysis by presenting estimates of equation (8), which exploit year and city variation for the purpose of identification, corresponding to a standard difference-in-differences (DiD) model. These estimates are presented in Section 6.1. Section 6.2 reports the results from the triple-differences (DiDiD) model of equation (9), which adds the control diseases to the setup. The findings from the event studies in equations (10) and (11) are reported in Section 6.3. Finally, our robustness analyses are unfolded in Section 6.4.

\subsection{Difference-in-differences model}

Table 2 reports the baseline results from estimating equation (8). We find negative estimates that are statistically significant at the one percent level in all the specifications. Specifically, the estimate in column (1) suggests that an opening of a TB dispensary reduced TB mortality per 1,000 people by 0.25 . Given that the average TB mortality rate was 1.31 , this corresponds to a reduction of about 19 percent. This is almost similar to the magnitude obtained using a log transformation in column (2). To get a sense of the magnitude, the average TB mortality per 1,000 people in the Danish cities fell by 1.32 from 1.65 in 1908 to 0.33 in 1939 . A comparison of the actual decline in the number of TB deaths in the cities from 1908 to 1939 with the counter-factual decline without the TB dispensaries suggests that they can explain 27 percent of this decline. ${ }^{35}$

\footnotetext{
${ }^{35}$ The counter-factual number of deaths is based on the estimate in column (1) of Table 2, calculated for the 75 cities constituting a balanced panel from 1908 to 1939.
} 
According to the SIR model, the estimating equation should also include a lagged dependent variable (see equation 2 in the Online Appendix). Nevertheless, our baseline estimate remains stable both in magnitude and statistical significance when we add the one-year lagged TB mortality rate (column 3) or up to five lags of the TB mortality rates (column 4). ${ }^{36}$ Finally, column (5) considers only pulmonary TB mortality, as this particular form of TB is where we expect to see an influence from the preventive actions of the dispensaries. ${ }^{37}$ We find a negative and statistically significant coefficient at the one percent level. Using the observed average of pulmonary TB, the magnitude of the estimate suggests that the opening of a dispensary reduces the pulmonary TB mortality rate by around 22 percent, which, as expected, is larger than the effect on all TB forms as reported in column (1).

\section{Table 2: Effect of TB dispensaries using city-by-year data on TB}

Placebo outcomes Table 3 reports the results when using mortality rates of cancer, influenza, pneumonia, accidents and suicides, scarlet fever, diphtheria, and polio as placebo-disease outcomes in equation (8). As it is only possible to obtain data on the placebo outcomes for the period 1901-1939, column (1) starts by showing that the effect on TB mortality remains negative, significant, and of the same magnitude for this restricted time period. Columns (2)-(8) next report the estimates for the placebo outcomes, and we see that the estimated coefficients are mostly negative, but small in numerical magnitude, and always statistically insignificant. For example, for pneumonia in column (4), which arguably is the disease most similar to TB, the estimated coefficient is not a precise zero, but it is 71 percent smaller in numerical magnitude than the baseline TB coefficient, which means that an opening of a dispensary reduced the pneumonia mortality rate by 5.1 percent. If anything, the evidence is suggestive of a negative effect on pneumonia albeit the coefficient is statistically insignificant, which, compared to the effect on TB is much smaller in magnitude. The coefficients on the remaining placebo diseases are even smaller in magnitude.

This pattern suggests that in the end the dispensaries mainly fulfilled their stated purpose of

\footnotetext{
${ }^{36}$ As we have time series for nearly 50 years, we are not strongly concerned about Nickell bias. This is confirmed when using the alternative Arellano-Bond estimator, which gives a similar result (available upon request).

${ }^{37}$ Pulmonary TB accounts for the bulk of the variation in the overall TB mortality rate; that is, the ratio of pulmonary TB to total TB is 77 percent in the sample from 1901 to 1939.
} 
preventing and reducing TB and indicates that our baseline estimate is not picking up a general trend in mortality. It also seems to suggest that competing risk and co-mortality are not relevant for this group of diseases. ${ }^{38}$ However, it might be that competing risk only materializes some years after the introduction of a dispensary and, as the DiD estimator measures the average effect for all the post-treatment years, this could result in insignificant DiD estimates. For this reason, we also estimate event studies for all the placebo diseases in order to check if this is indeed the case (see Section 6.3).

\section{Table 3: Placebo outcome regressions}

Child mortality, overall mortality, and fertility Table 4 investigates whether the dispensaries had any impact on child mortality, overall mortality, and fertility. The estimates, reported in columns (1) and (2), reveal that the rollout of the dispensaries reduced the child mortality rate by 11 percent, supporting the argument in Bütikofer and Salvanes (2015) that reducing TB benefited young children as well. This finding is also unsurprising in the light of the age distribution of TB mortality for Denmark in, for example, 1921-25 (see Online Appendix Figure A14), which shows that TB mortality for children below the age of five is as high as for adults of prime age.

Next, we investigate whether there is any impact on overall mortality (i.e., the crude death rate). Columns (3) and (4) report negative coefficients that are statistically significant at the five percent level. We see that dispensaries reduced the crude death rate by about four percent, which is substantially smaller compared to the effect on TB. This is very much in line with the simulation results from the modified SIR model in section 3 and, therefore, expected. These findings also suggest that the dispensaries reduced the disease death ratio for TB, which is confirmed in Online Appendix Table A4, where the disease death ratio for TB is the outcome of interest. $^{39}$

Previous research has argued that mortality changes might influence fertility patterns due to more women surviving to birth giving ages or replacement behavior (e.g., Acemoglu and Johnson,

\footnotetext{
${ }^{38}$ The literature has suggested that TB and influenza have strong co-morbidity. Yet Noymer (2009) investigates whether TB morbidity predicts dying from influenza using a sample of Union Army veterans from the 19th century, and finds a positive, yet statistically insignificant, relationship.

${ }^{39}$ The disease death ratio for TB is defined as the number of TB deaths divided by the total number of deaths.
} 
2007; Ager et al., 2017). Columns (5) and (6) show positive, but insignificant, estimates for the crude birth rate. One interpretation of these findings is that the effect from more women surviving to birth giving ages dominates possible replacement behavior, which would go in the opposite direction since more children now survive.

\section{Table 4: Effect of TB dispensaries using city-by-year data on other outcomes}

\subsection{Triple-differences model}

Table 5 reports the findings of estimating equation (9), which is the triple-differences model, using all the aforementioned control diseases. This type of model allows us to include additional fixed effects as compared to the previous DiD model. For example, city-by-year fixed effects account for all the variation that occurs between the different cities over time, such as processes of convergence or divergence in, for example, income, income inequality, or mortality; local political or institutional changes; migration; and pollution (e.g., Beach and Hanlon, 2016). Moreover, controlling for disease-by-city effects allows the basic mortality environment to be systematically different across the cities.

Column (1) shows an estimate that is quite similar to our baseline DiD estimate, that is,

$\hat{\beta}=0.21$ with standard error $=0.06$, so evaluating at the average, we find that an opening of a dispensary reduces the TB mortality rate by 20 percent. Columns (2) and (3) demonstrate that this quantification is robust to the inclusion of one/five lagged dependent variables. Overall, we conclude that these two different ways (i.e., DiD and DiDiD) of estimating the effect of the dispensaries on TB mortality yield the same conclusion, both in terms of sign and magnitude.

\section{Table 5: Effect of TB dispensaries using city-by-year-by-disease data}

\subsection{Event study analysis}

This subsection reports evidence indirectly supporting the identifying assumption that the TB mortality rate would have continued its pre-treatment path in the absence of a TB dispensary, by showing that there were no systematic trends prior to its introduction. Moreover, the subsequent dynamics, which is also revealed in these models, shows that the TB dispensaries had a permanent negative level effect on the TB mortality rate. 
For convenience, instead of reporting the results in regression tables, we plot the estimated $\beta_{j}$ 's from the event studies of the DiD and DiDiD models along with their 95 percent confidence intervals in Figure $6 .^{40}$ In both models, the estimated coefficients in the years preceding the TB dispensaries fluctuate non-systematically around zero, which supports the common pre-trend assumption. After the introduction, however, we observe a permanent downward shift in the level of the TB mortality rate. The estimated coefficients of equation (10) become significantly negative two years after the introduction of the TB dispensary and tend to become larger in absolute terms and more precisely estimated after more years have passed since the introduction. Although the confidence intervals of the coefficients estimated from equation (11) are wider, we observe a similar pattern, and the majority of the coefficients are statistically significant at the 10 percent level especially some years after establishment.

The estimated coefficient patterns from these event studies are line with our theoretical simulation, depicted in Figure 4, and so the theory supports that the full effect materializes relatively fast. In addition, this pattern is in line with estimates on the time to death for the pre-chemotherapy era. Goodman and Fuller (2015) report that the median time to death of untreated TB is 2.5 years. Tiemersma et al. (2011) estimate that the average time to death is 3 years. For Denmark the time to death from notification of pulmonary TB for the period 1925-34 was on average 14.3 months for males and 12.9 for females as reported by Lindhardt (1938), who analyzed around 40,000 notifications for this period. Given that TB takes time to develop, this is in line with the average suggested by Tiemersma et al. (2011). It should, however, be noted that nearly 30 percent die within 6 months of notification, and the medical literature suggests that some people develop TB within 1 to 3 years (Flynn and Chan, 2001) and that death can happen after a few weeks, as pointed out by Nagelkerke (2012). Moreover, there is substantial variance in how fast different age groups die. There is a significant number of infant (below one year) and child TB deaths and these groups die faster. The same is true for TB deaths in the group above age 65, who according to Lindhart (1937) died four months after notification on average.

Online Appendix Figures A18-A24 show the results from estimating event studies for all the placebo diseases in the DiD setup (i.e., equation 10). In general, we find no evidence of

\footnotetext{
${ }^{40}$ The regression tables are shown in Online Appendix Table A5.
} 
pre-treatment trends in any of these observables, and there seem to be no effects even after 10 years or more after the introduction of a dispensary, indicating that there is little competing risk or co-mortality between TB and our set of placebo diseases (even in the longer run).

\section{Figure 6: Event-study estimates of the impact on TB before and after TB dispensary}

\subsection{Robustness}

We have carried out a number of robustness checks based on both the DiD model and the DiDiD model. First, we perform a random placebo test, where we randomly shuffle the year of establishment of the TB dispensaries and reestimate the DiD and DiDiD models to exclude the possibility that we are capturing a spurious relationship. Second, we study explicitly if there are any spillover effects coming from the opening of a dispensary in a neighboring city. Third, we turn to other public health policies targeted at TB in the form of sanatoria and TB hospitals. Fourth, as poverty and inequality are possible determinants of TB mortality, we control for the pre-intervention income and a pre-intervention city-Gini coefficient. Fifth, we address treatment heterogeneity with respect to the initial TB rate, income, and inequality. Finally, we discuss a series of additional robustness checks reported in the Online Appendix.

Random placebo test We have investigated possible mis-specifications of the models caused by spurious relationships between the rollout of the dispensaries and the decline in TB by performing random placebo tests. Specifically, we randomly shuffle the year of commissioning of the dispensaries 2,499 times and obtain the placebo coefficients from re-estimating the DiD and the DiDiD models. Figure 7 shows the distributions of the placebo coefficients and the original coefficients (marked by vertical dotted lines) along with kernel density plots. The distributions resemble Gaussian curves centered at zero with the actual coefficients positioned to the far left. The areas to the left of the actual coefficients under the kernel distributions are 0.005 and 0.004 for the DiD and DiDiD models, respectively. ${ }^{41}$ This demonstrates that the true years of

\footnotetext{
${ }^{41}$ The kernel density functions are estimated using the Epanechnikov kernel and a bandwidth of 0.016 and 0.014 for the DiD and DiDiD models, respectively.
} 
commissioning of the TB dispensaries are necessary to produce the results and further suggests that our results are not spurious.

\section{Figure 7: The distribution of coefficients from random placebo tests}

Neighboring spillover effects It is possible that introducing a dispensary in one city had a negative spillover effect to another neighboring city's TB mortality rate if there is some (market/population) integration between the cities. While this might be an interesting finding in itself, this would change the interpretation of our baseline estimates, such that they should be interpreted as relative effects since the Stable Unit Treatment Value Assumption (SUTVA) would be violated in this case.

Table 6 reports the results from controlling for the opening of a dispensary in a neighboring city, where "neighbor" is classified as being a city within 10km (columns 1 and 2), 30km (columns 3 and 4 ), or $50 \mathrm{~km}$ (columns 5 and 6 ). We find that both the DiD and the DiDiD point estimates are (if anything) numerically larger compared to their baseline values, and they are all significant at the five percent level or more. In addition, the "neighbor" estimates are all negative, but not statistically significant; that is, there is only limited evidence of spillover effects from the opening of dispensaries in neighboring cities. We therefore conclude that the SUTVA condition is largely satisfied and if anything the baseline underestimates the total effect of the dispensaries.

\section{Table 6: Effect of TB dispensaries controlling for neighboring dispensaries}

Sanatoria and TB hospitals Table 7 investigates the robustness of our results to the commissioning of sanatoria and TB hospitals. There were around 80 of these institutions spread across the country during this time period, and most of them opened quite early. As most sanatoria were placed in the countryside (outside cities), we capture their influence on TB mortality by the presence of at least one sanatorium within $50 \mathrm{~km}$ of a city. We measure the impact of the TB hospitals by the presence of a TB hospital within the county of each city. This choice is motived by institutional reasons in the sense that an infected individual would only be referred to a TB hospital within his county of residence.

Columns (1)-(4) report the results for the sanatoria, while columns (5)-(8) report the results for the TB hospitals. In the DiDiD model, reported in the even-numbered columns, we assume 
that the sanatoria and TB hospitals only affect TB. The following results are worthwhile noticing: first, we see that both the DiD and the DiDiD estimates of the dispensaries remain relatively stable in magnitude and statistical significance and if anything, the numerical magnitude increases somewhat (columns 7 and 8). Second, we cannot whether sanatoria had any effect on TB mortality; the point estimates are both negative and positive, and too imprecisely estimated to say anything with just some degree of certainty. Third, the estimate on the interaction between the rollout of dispensaries and sanatoria is very close to zero and highly insignificant in both models (columns 3 and 4). This could suggest that isolation is not the main mechanism by which the dispensaries reduced TB mortality. On the other hand, it could also simply reflect that infected individuals had the liberty of choosing the sanatorium across the country that they liked the most. If this choice is largely unrelated to the distance from where they lived, we cannot measure the local impact of the sanatoria and their interaction with the dispensaries. Fourth, and finally, we see very little impact of the TB hospitals and their interaction with the dispensaries on TB mortality. Since we can be more sure here that we are able to measure their local impact (due to the institutional setup), the latter caveat is less likely to apply.

\section{Table 7: Effect of TB dispensaries controlling for commissioning of TB hospitals and sanatoria}

\section{Controlling for income, taxpayers per capita, and inequality before the intervention}

TB mortality could to some degree be biased towards the citizens with limited means who potentially suffered from malnutrition. This is a conceivable concern regarding the DiD analysis if the income level of the cities affected the rollout of the dispensaries. For example, richer places may have suffered less from the disease or could better attract nurses trained in treating TB. This is less of a concern for the DiDiD model, as we control for time-varying city fixed effects and time-varying disease fixed effects. Still, if income affected TB mortality more strongly as compared to other diseases, this would remain an issue. ${ }^{42}$ In the DiDiD model, we therefore make the very conservative assumption that pre-intervention variation in income and inequality only potentially influences TB, which is necessary to do, as these interactions would otherwise

\footnotetext{
${ }^{42}$ We note that this concern is being addressed somewhat by using similar infectious diseases, such as pneumonia and scarlet fever as controls which are also likely to be affected by income (see Online Appendix Table A6).
} 
be absorbed by the city-by-year fixed effects.

Columns (1) and (2) of Table 8 present estimates conditioning on the log tax income per capita (for the tax year 1904-05) interacted with a full set of time fixed effects, and in the DiDiD model also interacted with the prevent indicator. ${ }^{43}$ In columns (3) and (4) we show that the results are unchanged when we control for the 1904-05 taxpayers per capita interacted with a full set of time fixed effects as a proxy for labor force participation, which could reflect differences in the labor market. In the tax year 1918-19, the income distribution of the citizens liable to pay income taxes became available, and it becomes possible to calculate a Gini coefficient for each city. Columns (5) and (6) include the Gini coefficient of 1918-19 interacted with a full set of time fixed effects (and in the DiDiD model also interacted with the prevent indicator), and exclude cities that adopted a dispensary before 1920 to control for inequality within the cities. ${ }^{44,45}$ Columns (7) and (8) control simultaneously for pre-intervention income, taxpayers per capita, and inequality. As seen from the reported estimates, even when we control for these pre-intervention variation interactions, the results are similar to the baseline.

\section{Table 8: Effect of TB dispensaries controlling for pre-intervention income, taxpayers per capita, and inequality}

Treatment heterogeneity Table 9 explores possible treatment heterogeneity in the dimensions of initial TB mortality, population density, tax income per capita, and within-city income inequality. Columns (1) and (2) include an interaction term between the dispensary indicator and the initial (standardized) 1890 TB mortality rate in equations (8) and (9), respectively. Likewise, columns (3) and (4) include the interaction between (standardized) 1890 population density and the dispensary indicator. Columns (5) and (6) include an interaction term between

\footnotetext{
${ }^{43}$ Denmark introduced income taxation in 1903 (Aidt and Jensen, 2009), for which reason taxable income is available from 1904 onwards. According to Philip (1955, p.57), the main principles remained the same throughout the period we study. There was an exemption of 800 Danish kroner in Copenhagen and of 700 Danish kroner in the market towns. The income tax was progressive and rates were initially very low (Seligman: 1908, p.83) but increased over time, see Philip (1955, p.56).

${ }^{44}$ For cities included in the datas et later than 1904 and 1918, we use the income per capita, taxpayers per capita, and the Gini coefficient in the year they are added to the data. None of the cities added to the dataset, had a TB dispensary initially. We cannot observe people with income below 800 Danish kroner a year, as they were not liable to pay taxes

${ }^{45}$ We do not include the contemporary income per capita and the Gini coefficient because TB mortality and income or inequality are likely jointly determined.
} 
the dispensary indicator and the (standardized) 1904 log tax income per capita. Finally, in columns (7) and (8), cities that adopted a dispensary before 1920 are excluded from the sample, and an interaction term between the dispensary indicator and the (standardized) 1918 Gini coefficient is included. ${ }^{46}$

Reassuringly, compared to the baseline estimates, the point estimate of the TB dispensary remains unchanged in all specifications. The interaction between the dispensary and the initial TB mortality rate is negative and significant in both the DiD and the DiDiD models, implying that a one standard deviation increase in the initial TB mortality rate increases the effect of the dispensary by 88 percent in the case of the DiD estimate. It suggests that TB dispensaries were less effective in areas with low initial TB mortality, which we use in Online Appendix Table A7 to propose a modified treatment measure (see below). We find small positive estimates on the initial population-density interaction. The interaction between the dispensary and the initial log tax income per capita enters positively and significantly into both models suggesting that cities with a higher initial income benefited less from a dispensary. This finding can be explained by the hypothesis that higher income leads to a better state of nutrition thereby decreasing the likelihood of dying from TB. Lastly, the interaction term with the initial Gini coefficient is small and insignificant, and hence within-city income inequality does not seem to affect the treatment effect of the dispensaries. Thus, the effect does not vary with inequality within the city, but rather with income inequality between the cities.

\section{Table 9: Treatment heterogeneity of TB dispensaries}

Additional robustness checks Finally, we briefly mention our battery of additional robustness checks, reported in the Online Appendix. First, it is possible that the general information on avoiding TB affected other airborne infectious diseases due to better hygienic practices of not spitting on the street or coughing in public. While the placebo outcome regressions suggest that there are limited spillover effects to other (airborne) diseases, one might (at the least ex-ante) believe that there are spillover effects on scarlet fever and pneumonia. According to Jayachandran et al. (2010), these two diseases are the infectious diseases that bear the most

\footnotetext{
${ }^{46}$ For cities included in the data set later than 1890 , we use the TB mortality rate in the year they are added to the data.
} 
similarities with TB. We address this issue in two alternative ways. First we limit the control diseases to the most similar diseases; scarlet fever and pneumonia. If the dispensaries only affected TB through general information that potentially could affect similar infectious diseases, we should find a smaller effect using this subset of control diseases. Second, we limit the panel by excluding all infectious diseases, using only cancer and accidents/suicides as controls, which are unlikely to be affected by the intervention. Online Appendix Table A6 shows DiDiD estimates that are very similar to the baseline, using these alternative subsets of control diseases.

Online Appendix A7 shows the basic DiD and DiDiD estimates, using two alternative treatment measures. First, instead of the baseline indicator, we use a treatment measure that takes on the value zero before the intervention and years since the adoption of the dispensary afterwards. We find negative and significant estimates, which, along the lines of our event studies, suggests that the effect is (weakly) increasing over time. Second, we have tried to exploit the five-year average TB mortality rate just prior to the intervention as a measure of treatment intensity, similar in spirit to the basic idea of Bleakley (2007); that is, cities with higher levels of pre-intervention TB stood to gain more from the opening of a dispensary. Also, with this second measure, we obtain strong negative effects.

The BCG vaccine became widespread in Denmark in the 1940s, but the remote island of Bornholm experimented with the vaccine from 1936. Online Appendix Table A8 demonstrates that our results are robust to excluding the cities on Bornholm and limiting the panel to $1935 .{ }^{47}$ Given that the BCG vaccine is less effective against pulmonary TB - the main component of TB mortality - it is not surprising that the results are largely unaffected. Moreover, limiting the panel to 1935 also excludes the possibility that our results are influenced by the rollout of a universal home-visiting program for mothers and their infants starting in Denmark in 1937 (Wüst, 2012).

Online Appendix Table A9 documents that our findings are generally robust to excluding Copenhagen (the capital and largest city), the five largest cities, and all cities that adopted a dispensary before $1920 .^{48}$ Online Appendix Table A10 further shows that the results are

\footnotetext{
${ }^{47}$ The Medical Report for 1940 describes that special efforts were being made on Bornholm from 1936 onwards. 1159 people were vaccinated in this period.

${ }^{48}$ In 1890 and in 1901 the five largest cities were Copenhagen, Frederiksberg, Aarhus, Odense, and Aalborg, with 1901 populations of $378,235,76,231,51,814,40,138$, and 31,457. The sixth and seventh largest cities were Horsens and Randers with populations of 22,243 and 20,057 respectively.
} 
robust to controlling for the lag of log population, including the log of the initial population interacted with time fixed effects, weighting the estimation by the log of the initial population, and including a city-specific linear time trend. While we still obtain a negative coefficient when including city-specific linear time trends, the effect is only borderline significant. Yet, when we use the log of the TB rate amounting to constant growth in the TB rate, the coefficient is negative (-0.14) and significant at the ten percent level with a p-value of 0.054 and similar to the estimate without time trends (-0.17). We therefore conclude that the result in the level model is more likely to reflect a precision issue. We also note that in the baseline DiDiD settings, the linear trend is a special case of the non-parametric city-specific trend.

An additional concern could be that the effect from TB dispensaries is confounded by other (public) measures against infectious diseases at the time. To address this, we have controlled for the commissioning of waterworks in the cities, as clean water has been emphasized as an important public health intervention at this time, affecting mortality by improving sanitation and water supply (Cutler and Miller, 2005, Alsan and Goldin, 2015). TB is not a water-born disease; however, clean water could have general implications for overall mortality. As revealed by Online Appendix Table A11, this does not change our findings. ${ }^{49}$ We also control for the establishment of associations that provided cheap housing for workers, known as housing associations. We do so as TB infection is more likely to occur if housing conditions cause people to live in a crowded environment (Clark et al., 2002). Specifically, we control for the year of the establishment of the first housing association in a city. We find that this has little effect on results; see Online Appendix Table A11.

The system of TB dispensaries in Denmark was organized around larger central TB dispensaries with branches in different cities (Holm, 1946a). While the two types of branches performed similar tasks, the main dispensaries would often be led by specialized doctors, who also often worked at TB hospitals (Holm, 1946a). To analyze whether the main dispensaries had a different effect than their branches, we split the TB dispensary indicators in equations (8) and (9) into an indicator equal to one after the introduction of a main dispensary in a given city and a similar indicator for the introduction of a branch dispensary. We present the results in Online

\footnotetext{
${ }^{49}$ The first waterworks were commissioned in Odense in 1853, and by 1890 the five largest cities could all provide its citizens with clean water from waterworks. In 1890, 25 of the cities in the sample had waterworks, by 1901, 36 cites had waterworks, and by 1939, 85 of the 87 cities had waterworks.
} 
Appendix Table A12. We cannot reject that the effects of the main and branch dispensaries are statistically equal and the magnitude of the estimated effects are similar to the baseline results.

Online Appendix Table A13 documents that the results are robust to reducing the sample to the 74 cities that constitute a balanced panel, and extending the sample period from 1939 to 1946; the year after the last dispensary was established. The absolute magnitude of the coefficient on TB dispensary is somewhat reduced when we extend the year to 1946, although it is still highly statistically significant.

Finally, we note that pasteurization has been argued to have negative impacts on Bovine TB (BTB), scarlet fever, and diphtheria since milk would contain bacteria from these diseases (Wilson, 1943; Jensen, 2002). However, pasteurization would kill the bacteria and, therefore, prevent transmission. Moreover, as argued by Olmstead and Rhode (2004, p.768), BTB was mainly associated with non-pulmonary TB and had a different mode of transmission. Evidence by Jensen et al. (1940) shows that in many towns there was no BTB, and the percentage was below 10 for all age groups. Above we found a strong effect on pulmonary TB, which suggests that our results are not explained by pasteurization and BTB. ${ }^{50}$ Moreover, our placebo regressions reveal no impact on scarlet fever and diphtheria, which would also be impacted by pasteurization. We also note that results are robust to ending the sample in 1935, at which time BTB was still highly prevalent. Indeed, the eradication of BTB only gathered momentum after 1935, and infected herds still counted 60-80 percent out of total herds in 1937; see Groth-Petersen et al. (1959). We therefore conclude that our results are unlikely to be explained by BTB eradication and pasteurization.

\section{Monthly analysis}

This section unfolds our monthly mortality data set. Figure 8 starts the investigation by displaying the monthly development in the number of TB deaths by urban and rural areas in panels A and B, respectively. As in the annual TB series in Figure 1, we see a general decline in TB mortality throughout the period in both areas. However, there also appears to be seasonality

\footnotetext{
${ }^{50}$ The decline in non-pulmonary TB cannot alone explain the estimated effect of the TB dispensaries. Running the difference-in-differences regression on the non-pulmonary TB rate results in a small coefficient of -0.046 which is significant at the 5 percent level (standard error $=0.023$ ).
} 
during the year in the number of TB deaths, although the variance seems to be dampening over time. For the urban areas in the 1920s, the number of TB deaths was relatively high during winter and spring, whereas in the rural areas the number of TB deaths generally peaked during spring. These seasonal patterns across the two areas persist until the start of the 1930s, after which they start to dissipate. We refer to Online Appendix Figure A15 for more detailed representations of the monthly TB data.

If there is any effect of the observed seasonal pattern on our baseline annual DiD and DiDiD estimates, the following strategy is designed to explicitly take this matter into account. In particular, using information on the exact month and year of establishment, we now test if the effect of the rollout of the dispensaries can be traced in terms of reductions in the monthly number of TB deaths, while exploiting other diseases as controls in the spirit of our previous DiDiD model. Among other things, this type of model has the advantage of being able to eliminate changing seasonal variation over time, and it allows us to zoom in on the dynamics effects of opening a dispensary. We begin by estimating the following model:

$$
M_{d, u, t, m}=\beta \text { Dispensary }_{u, t, m} \times \text { Prevent }_{d}+\phi_{d, u, t}+\phi_{u, t, m}+\phi_{d, u, m}+\phi_{d, t, m}+\varepsilon_{d, u, t, m}
$$

where $M_{d, u, t, m}$ denotes the total number of deaths of disease $d$ in area $u$ (i.e., urban/rural) in year $t$ in month $m$. Our geographical unit of analysis is now urban/rural area,s and Dispensary ${ }_{u, t, m}$ therefore stacks (or counts) the number of dispensaries rolled out up until month $m$ in year $t$.

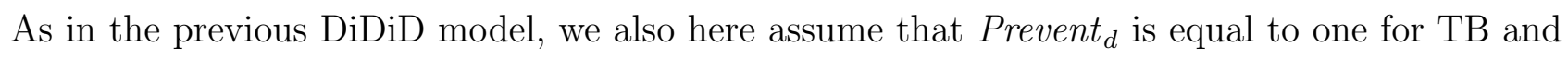
zero otherwise. This model allows us to control non-parametrically for disease-area-year fixed effects $\left(\phi_{d, u, t}\right)$, area-year-month fixed effects $\left(\phi_{u, t, m}\right)$, disease-area-month fixed effects $\left(\phi_{d, u, m}\right)$, and disease-year-month fixed effects $\left(\phi_{d, t, m}\right)$. For example, $\phi_{u, t, m}$ controls for possible changing seasonal variation across time and areas, which Figure 8 suggests to be the case. The error term $\left(\varepsilon_{d, u, t, m}\right)$ is allowed to cluster at the disease-by-area level. The diseases (i.e., causes of death) included in this panel are: tuberculosis, measles, scarlet fever, typhoid fever, alcohol, accidents, suicides, diphtheria, puerperal fever, pneumonia, and cancer, which are the diseases that are consistently available throughout the sample period from 1920 to 1939. It is important to note that the dispensaries were rolled out both in urban and rural areas, and so one cannot think of 
the rural areas as being in the control group only.

In addition to estimating equation (12), we also estimate a model with 48 lag dummies, where a dummy variable measures the change in the total number of dispensaries a given number of months ago. For example, if lag dummy 24 takes on the value two, it means that exactly 24 months ago two additional dispensaries were established in that area. ${ }^{51}$ While our annual event study suggested an effect after a couple of years, we can use this model to trace out the dynamics within years; that is, we can ask how many months it on average take before we see a decrease in the number of TB deaths after the establishment of a dispensary. The same interaction fixed effects as above are included in this model.

Table 10 reports the results from estimating equation (12), and Figure 9 displays the estimates along with standard errors from the proposed lag-dummy model. We find that $\hat{\beta}=-1.77$ with standard error $=0.59$. Using that the average number of TB deaths per month is around 104, this estimate suggests that for one additional dispensary, the total number of TB deaths lower by about 1.7 percent. The results from the dummy-lag model presented in Figure 9 reveal an average response time of about 17-19 months. Thus, only after such a time period do we find a measurable effect in these aggregate series of monthly TB mortality following the opening of a dispensary.

\section{Figure 8: TB deaths per month from 1920 to 1939 in urban and rural areas Table 10: Effect of the TB dispensaries using urban/rural-by-month-by-disease data}

\section{Figure 9: Event-study estimates of the impact on TB of the change in the number of TB dispensaries 0 to 48 months after the change}

\section{Mechanisms}

While the robustness analysis already showed that our baseline findings are not likely to be confounded by sanatoria and TB hospitals, and there seems to be little evidence suggesting any

\footnotetext{
${ }^{51}$ We coded this model differently compared to our annual event study due to the fact that in our monthly analysis we stack the number of dispensaries, since the geographical variation here is limited to two areas (i.e., urban and rural).
} 
kind of interaction effect, this section exploits information on the number of patients admitted to these two institutions across counties over time to further consider the possible mechanisms.

Given the stated purpose and policies of the dispensaries, our baseline finding can be interpreted as an effect of a combination of personalized information provision and isolation of individuals in sanatoria and TB hospitals. As also emphasized in Section 3, data on the (institutionalized) isolation rate provide a possible way to investigate the relative importance of these two mechanisms, as one would expect the isolation rate to increase if the baseline effect is driven only by isolation. ${ }^{52}$

To obtain graphical evidence on this, we plot the number of patients in sanatoria, TB hospitals and the number of TB dispensaries in Figure 10. While all variables have a tendency to increase over time, we note that after the large increase in the number of TB dispensaries in the 1930s, the number of patients in both institutions tended to fall or stagnate. This suggests that isolation is not the only mechanism at play.

To investigate this more formally, we now estimate the following model:

$$
q_{i, t}^{v \in V}=\beta_{v} \text { Dispensary }_{i, t}+\alpha_{i}+\gamma_{t}+\varepsilon_{i, t}^{v},
$$

where $q_{i, t}^{v \in V}$ is the log number of admitted patients or the log number of admitted patients per capita (i.e., isolation rate) in county $i$ in year $t$. The patients can either be admitted to a sanatorium or a TB hospital $(v \in V)$. Since our unit of analysis is now at a higher level (i.e., county instead of city), Dispensary $y_{c, t}$ stacks (or counts) the number of dispensaries in the county in a given year, $\alpha_{i}$ and $\gamma_{t}$ are county and year fixed effects, and $\varepsilon_{i, t}^{v}$ is the error term. Note that the data section describes how we allocated the collected sanatorium- and TB hospital-level patient data to the counties.

Table 11 reports the results from estimating equation (13), where columns (1) and (2) report the sanatoria results, and columns (3) and (4) report the TB hospital results. We see that the number of sanatoria patients decreases when the local number of dispensaries increases: the isolation rate declines by 10 percent for one extra dispensary in the county (column 2), and column (1) simply shows that this effect is not driven by the denominator (i.e., county

\footnotetext{
${ }^{52}$ This statement is generally not true, but it is in fact the case under plausible assumptions.
} 
population size), but rather by the number of admitted patients. This evidence indicates that the TB incidence rate is in fact decreasing and that our baseline estimate cannot mainly be driven by isolation, which indirectly suggests that information provision was more important. As seen from the two remaining columns of the table, we find no evidence of an effect on the number of patients in TB hospitals.

\section{Figure 10: Number of patients in sanatoria and TB hospitals and number of TB dispensaries}

\section{Table 11: Effect of TB dispensaries on patients in sanatoria and TB hospitals}

\section{Cost-benefit analysis and spillover productivity effects}

This section first evaluates the direct costs and benefits of the 38 dispensaries commissioned from 1908 to 1939, after which we study whether the dispensaries had any (indirect) productivity effects. The baseline DiD estimate from column (1) in Table 2 suggests that the dispensaries averted 7,199 deaths over the period 1908-1939 with a 95 percent confidence interval spanning from 6,123 to 8,275 ; see Table 12 . Yet the number of lives saved is uninformative unless we obtain information on which age groups were saved. From 1921 the Cause of Death Statistics provides country-wide data on the TB mortality age distribution. We employ the age distribution of TB mortality in the five-year period 1921-25 as the counterfactual distribution of TB mortality without the prevention efforts of the dispensaries. By weighting the age-specific life expectancy in each year from 1908 to 1939 by the assumed age distribution of TB mortality, we get a measure of the life years saved for the average averted TB death for each year. ${ }^{53}$ The total life years saved from 1908 to 1939 are hereby estimated to be 284,403 years (95 percent confidence interval; 241,891 to 326,915$)$.

The annual reports of the National Association provide accounts of the expenditures of nine of the TB dispensaries in various years between 1912 and 1939. ${ }^{54}$ Using the average value of the yearly per capita costs of the nine dispensaries in 2015 US dollars to value the total costs of the

\footnotetext{
${ }^{53}$ The life expectancy in Denmark is obtained from the Human Mortality Database; see http: //www . mortality . org/.

${ }^{54}$ We have expenses for the cities of Bogense, Kerteminde, Odense, Randers, Silkeborg, Slagelse, Vejle, Viborg, and Aarhus; see Nationalforeningen til Tuberkulosens Bekæmpelse (1914-1940).
} 
dispensaries that operated from 1908 to 1939 amounts to 19 million dollars. This suggests that the cost of saving a life amounted to 2,668 dollars and the cost of saving a life year equalled 68 dollars (95 percent confidence intervals; 3,137 to 2,321 and 79 to 59, respectively).

With a cost per life year saved as low as 68 dollars there is little doubt that the benefits of the dispensaries must outweigh the costs, but it is still interesting to get a sense of the magnitude of the benefits. The newest estimates on the value of a life year in Denmark today is just above 190,000 dollars. ${ }^{55}$ However, it is well established both theoretically and empirically that the value of life should be viewed as a normal good in the sense that it increases with income (Hammitt and Robinson, 2011). Studies investigating the relationship between the value of life and income in Denmark are unavailable. We therefore turn to the study of the United States by Costa and Kahn (2004). They estimate that the elasticity of the value of a life with respect to the per capita gross national product is between 1.5 and 1.7 based on data from 1940 to 1980. To be conservative, we use the upper bound of this estimate and the growth rate of income per capita in Denmark from 1908 to 2007, which implies a value of a life year in 1908 of 6,977 dollars. ${ }^{56}$ Based on the estimate of the 1908 value of a life year, the total benefit of the dispensaries amounts to 1,984 million dollars (95 percent confidence interval; 1,688 to 2,281). As shown in Table 11, this adds up to a social rate of return of 103 to 1 (95 percent confidence interval; 88:1 to 119:1). This result suggests that a policy based on information provision has low cost and potentially large benefits even when TB cannot be treated as would be the case if the disease environment was dominated by multi-drug resistant TB. This is in contrast with policies that include costly treatment of multi-drug resistant TB, which reduces benefit-cost ratios (e.g. Jamison et al., 2013).

\section{Table 12: Costs and benefits of TB dispensaries}

While the economic benefits of the dispensaries clearly outweigh their costs, it is also possible that the reduction in the incidence and mortality of TB had indirect positive effects on average

\footnotetext{
${ }^{55}$ See the Danish Environmental Economic Council: www.dors.dk/files/media/rapporter/2016/M16/m16.pdf.

${ }^{56}$ The calculated value of a life year in 1908 is derived from the CES function $V O L Y_{1908}=$ $\operatorname{VOL} Y_{2015}\left(Y_{1908} / Y_{2015}\right)^{\epsilon}$, where $\epsilon$ is the income elasticity, $V O L Y_{t}$ is the value of a life year, and $Y_{t}$ is the income per capita, where the subscript $t$ denotes the year. Income per capita data are from the Maddison Project; see http://www.ggdc.net/maddison/maddison-project/home.htm. Because of data limitations, we use the ratio $\left(Y_{1908} / Y_{2007}\right)^{\epsilon}$ to deflate the value of a life year in 2015, as the Maddison Project only provides income per capita data up until 2010, and according to Statistics Denmark, GDP in Denmark in 2007 was at the highest level before the financial crisis. Income per capita in Denmark grew by 606 percent from 1908 to 2007.
} 
working productivity. Therefore, along the lines of previous studies on how health interventions influence economic productivity, human capital, etc. (e.g., Bleakley, 2007; Bütikofer and Salvanes 2015), we finally study whether the dispensaries affected working productivity. For this purpose, we digitized city level annual tax income (per taxpayer) from 1904 to 1939. Table 13 presents the results from regressing log tax income per taxpayer on the rollout of the dispensaries (using our baseline treatment measure and years since adoption) as well as city and year fixed effects. The baseline effect, reported in column (1), is significant at the 10 percent level, and the point estimate suggests that an opening of a dispensary increases income by about 3 percent. Comparing this estimate with the effect on TB mortality (column 2 of Table 2) indicates an income-TB mortality elasticity of around $-0.16 .{ }^{57}$ Next, column (2) exploits years since the dispensary opened as the treatment measure, implying that the estimated coefficient measures how the opening of a dispensary influenced the annual growth rate of income afterwards. Our estimate, which is now significant at the one percent level, reveals that a dispensary increases the annual income growth by 0.4 percentage points. The increased precision of the latter estimate reflects that the income effect takes some years to materialize and is generally increasing over time. We think that the most plausible interpretation of the positive reduced-form income estimates is that the reduction in TB (due to the dispensaries) lowers the annual number of sick days and thus increases productivity.

\section{Table 13: Productivity effects of TB dispensaries}

\section{Concluding remarks}

This study has shown that the introduction of TB dispensaries reduced TB mortality. This finding holds in both DiD and DiDiD setups. In both models, we provide indirect support of the main identifying assumption by showing that trends are parallel prior to treatment and a series of robustness checks. We also demonstrated that an analysis of monthly TB deaths confirms our baseline finding.

We show that the most likely interpretation of these findings is that dispensaries provided

\footnotetext{
${ }^{57}$ This elasticity number arises from the ratio of the "reduced form" (in Table 13) to the the "first stage" (in Table 2).
} 
personalized information on how to avoid transmitting and contracting TB. Our results also grant an important role to public health for the observed decline in TB prior to the advent of modern medicine. Yet, we acknowledge that increased income and nutrition, as stressed by McKeown (1976), could have been important as well, and our research is not designed to answer the question of whether the TB dispensaries had a stronger impact than improved nutrition. In addition, we note that other public health institutions such as TB hospitals and sanatoria seem to have had little effect on the development of TB over this period. While our study does not as such aim to evaluate these other types of TB institutions, these results can be seen as suggestive that not all public health institutions mattered equally.

We also show that the dispensaries were very cost effective compared to other public health interventions previously evaluated in the literature, and we take our analysis one step further (compared to these studies) by demonstrating positive spillover effects to working productivity. To our mind, the most plausible interpretation of these positive productivity effects is that when TB declines, workers have fewer sick days because of reduced TB infection.

As TB dispensaries were in use in many other countries, our study arguably has high historical external validity. Our research also provides some reason for believing that TB dispensaries could be an important and relatively cheap public intervention for combating TB in developing countries in which the disease is still highly prevalent. This knowledge is important as drug resistant strains have come into existence. However, we also acknowledge that transplanting TB dispensaries to developing countries is not a trivial task. 


\section{References}

Acemoglu, D., Johnson, S., 2007. Disease and development. Journal of Political Economy, 115(6), 925-985.

Adda, J., 2016. Economic activity and the spread of viral disease: evidence from high frequency data. Quarterly Journal of Economics, 31(2), 891-941.

Ager P., Hansen, C.W., Jensen, P.S., 2017. Fertility and Early-Life Mortality: Evidence from Smallpox Vaccination in Sweden. Journal of the European Economic Association, (forthcoming).

Aidt, T.S., Jensen, P.S., 2009. The taxman tools up: an event history study of the introduction of the personal income tax. Journal of Public Economics, 93(1), 160-175.

Alsan, M., Goldin, C., 2015. Wathershed in infant mortality: The role of effective water and sewerage infrastructure, 1880 to 1915. NBER working paper 21263.

Bailey, M.J., Goodman-Bacon, A., 2015. The War on Poverty's Experiment in Public Medicine: Community Health Centers and the Mortality of Older Americans. American Economic Review 105(3), 1067-1104.

Beach B., Hanlon, W.W., 2016. Coal Smoke and Mortality in an Early Industrial Economy. Economic Journal, forthcoming.

Bleakley, H., 2007. Disease and development: evidence from hookworm eradication in the American South. Quarterly Journal of Economics, 122(1), 73-117.

Becker, G. S.,Philipson, T.J., Soares R.R., 2005. The quantity and quality of life and the evolution of world inequality. The American Economic Review, 95(1), 277-291.

Bütikofer, A., Salvanes, K., 2015. Disease control and inequality reduction: Evidence from a tuberculosis testing and vaccination campaign. Unpublished working paper.

Bureau of Labor, 1912. Care of tuberculosis wage earners in Germany. Bulletin of the United States Bureau of Labor, 101, 1-182. 
Brimnes, N., 2008. BCG vaccination and WHO's global strategy for tuberculosis control 1948-1983. Social Science and Medicine, 67, 863-873.

Clark, M., Riben, P., Nowgesic, E., 2002. The association of housing density, isolation and tuberculosis in Canadian First Nations communities. International Journal of Epidemiology, 31, 940-945.

Clay, K., Troesken, W., Haines, M., 2014. Lead and Mortality. Review of Economics and Statistics, 96(3), 458-470.

Costa, D.L., Kahn, M.E., 2004. Changes in the value of life, 1940-1980. Journal of Risk and Uncertainty, 29(2), 159-180.

Cutler, D., Deaton, A., Lleras-Muney, A., 2006. The determinants of mortality. Journal of Economic Perspectives, 20(3), 97-120.

Cutler, D.M., Meara, E., 2004. Changes in the age distribution of mortality over the twentieth century. In: Wise, D.A. (Ed.), Perspectives on the Economics of Aging. University of Chicago Press, pp. 333-365.

Cutler, D, Miller, G., 2005. The role of public health improvements in health advances: The twentieth-century United States. Demography 42(1), 1-22.

Daniels, M., 1949. Tuberculosis in Europe during and after the second world war. British Medical Journal, 2(4636), 1065-1072.

Deaton, A., 2006. The Great Escape: A Review of Robert Fogel's The Escape from Hunger and Premature Death, 1700-2100. Journal of Economic Literature, XLIV, 106-114.

Dormandy, T., 1999. The White Plague. The Hambledon Press, London, UK.

Fisher, I., 1912. A revised stimate of the economic cost of tuberculosis. Transactions of the annual meeting, 8, 123-139.

Faber, K., 1938. Tuberculosis and nutrition. Acta Tuberculosea Scandinavica, 12, 287-334. 
Ferrie, J., Troesken, W., 2008. Water and Chicago's mortality transition, 1850-1925. Explorations in Economic History, 45, 1-6.

Flynn, J., Chan, J., 2001. Tuberculosis: Latency and Reactivation. Infection and Immunity, 69(7), 4195-4201.

Fogel, R. W., 1994. Economic Growth, Population Theory, and Physiology: The Bearing of Long-Term Processes on the Making of Economic Policy. American Economic Review, 84, 369-395.

Fogel, R., 1997. New Findings on Secular Trends in Nutrition and Mortality: Some Implications for Population Theory. In Mark R. Rosenzweig and Oded Stark, (Eds.), Handbook of population and family economics, Vol. 1A. Arnsterdam: North-Holland.

Frith, J. 2014. History of Tuberculosis. Part 1 - Phthisis, consumption and the White Plague. Journal of Military and Veterans' Health, 22(2), 29-35.

Gersovitz, M., Hammer, J.S., 2004. The economical control of infectious diseases. Economic Journal, 114(492), 1-27.

Goodman, C.C., Fuller, K., 2015. Pathology: Implications for the physical therapist, 4th edition. Elsevier Saunders, St. Louis, Missouri.

Groth-Petersen, E., Knudsen, J., Wilbek, E., 1959. Epidemiological basis of tuberculosis eradication in an advanced country. Bulletin of World Health Organization, 21, 5-49.

Hammitt, J.K., Robinson, L.A., 2011. The income elasticity of the value per statistical life: Transferring estimates between high and low income populations. Journal of Benefit-Cost Analysis, 2(1), 1-27.

Hansen, C.W., 2013. Life expectancy and human capital: Evidence from the international epidemiological transition. Journal of Health Economics, 32(6), 1142-1152.

Hemskerk, D., Caws, M., Marais, B., Farrar, J., 2015. Tuberculosis in adults and in children. SpringerBriefs in Public Health e-book. 
Hollingsworth, A., 2014. Controlling TB in a World without Antibiotics: Isolation and Education in North Carolina, 1932-1940. Working paper.

Holm, J., 1946a. Tuberculosis control in Denmark. Public Health Reports, 61(40), 1426-1443.

Holm, J., 1946b. BCG vaccination in Denmark. Public Health Reports, 61(36), 1298-1315.

Høy-Nielsen A., 2012. Tuberkulose som folkesygdom 1900-1962, Esbjerg Byhistoriske Arkiv, Denmark.

Jayachandran, S., Lleras-Muney, A.,Smith, K.V., 2010. Modern medicine and the twentieth century decline in mortality: Evidence on the impact of sulfa drugs. American Economic Journal: Applied Economics, 2(2), 118-146.

Jamison, D.T., Jha, P. Laxminaryan, R., Ord, T., 2013. Infectious Disease, Injury and Reproductive Health. In Lomborg, B. (ed.) Global Problems, Smart Solutions: Cost and Benefits. Cambridge University Press, Cambridge, United Kingdom.

Jensen, K., 2002. Bekæmpelse af infektionssygdomme - Statens Serum Institut 1902-2002. Nyt Nordisk Forlag Arnold Busck, Copenhagen, Denmark.

Jensen, K.A., Lester, V., Tolderlund, K., 1940. The frequency of bovine infection among tuberculous patients in Denmark. Acta Tuberculosea Scandinavica, 14, 125-157.

Johansen, H.C., 2002. Danish population history, 1600-1939. University Press of Southern Denmark, Odense, Denmark.

Kermack, W.O., McKendrick, A.G., 1927. A Contribution to the Mathematical Theory of Epidemics. Proceedings of the Royal Society A: Mathematical, Physical and Engineering Sciences. 115(772), 700.

Lawrence, C., 2006. Continuity in crisis, 1914-1945. Chapter 3 in Bynum, W.F., Hardy, A., Jacyna, S., Lawrence, C., Tansey, E.M. (eds.). The Western medical tradition: 1800-2000. Cambridge University Press, New York, New York.

Lindhardt, M., 1939. The statistics of pulmonary tuberculosis in Denmark, 1925-34. Munksgaard, Copenhagen, Denmark. 
Madajewicz, M., Pfaff, A., van Geen, A., Graziano, J., Hussein, I., Momotaj H., Sylvi, R., Ahsan, H., 2007. Can Information Alone Change Behavior? Response to Arsenic Contamination of Ground-water in Bangladesh. Journal of Development Economics, 84(2), 731-754.

Marais, B.J., Donald, P.R., Gie, R.P., Schaaf, H.S., 2005. Diversity of disease in childhood pulmonary tuberculosis. Annals of Tropical Paediatrics, 25(2), 79-86.

McKeown, T., 1976. The modern rise of population. Edward Arnold Ltd, Great Britain.

Nagelkerke, N., 2012. Courtesans and consumption: How sexually transmitted infections drive tuberculosis epidemics. Eburon Academic Publishers.

Nelson, L.J., Wells, C.D., 2004. Global epidemiology of childhood tuberculosis. The International Journal of Tuberculosis and Lung Disease, 8(5), 636-647.

Nordhaus, W.. 2002. The Health of Nations: The Contribution of Improved Health to Living Standards," in The Economic Value of Medical Research. Kevin Murphy and Robert Topel, eds. Chicago: University of Chicago Press.

Noymer, A., 2009. Testing the influenza-tuberculosis selective mortality hypothesis with Union Army data. Social Science \& Medicine, 68, 1599-1608.

O'Grada, C., 2015. The challenge of antimicrobial resistance: lessons from the past for the present and for the future. Global Perspectives Series: Paper 2. Centre for the Competitive Advantage in the Global Economy, University of Warwick.

O'Grada, C., 2016. "Cast back into the dark ages of medicine"? What the past can tell us about antimicrobial resistance. American Journal of Medical Resarch, 3(1), 142-173.

Olmstead, A.L., Rhode, P.W., 2004. An impossible undertaking: The eradication of bovine tuberculosis in the United States. Journal of Economic History, 64(3), 734-772.

Permin, G.S., 1912. Om en ny Virksomhed for Sygeplejersker (Tuberkulose-Stationerne). Tidsskrift for sygepleje, særtryk, 1912.

Philip, K., 1955. Skattepolitik. Gyldendal, Copenhagen, Denmark. 
Porter, D., 1999. Health, civilization and the state: A history of public health from ancient to modern times. Routledge, London, England.

Preston, S.H., 1975. The changing relation between mortality and level of economic development. Population Studies, 29, 231-248.

Preston, S.H., 1996. American longevity: Past, present, and future. Syracuse University Policy Brief no. 7/1996. Distinguished Lecturer in Aging Series. Syracuse, N.Y.: Syracuse University, Center for Policy Research.

Rouzier, V.A., Oxlade, O., Verduga, R., Gresely, L. Menzies, D., 2010. Patient and family costs associated with tuberculosis, including multidrug-resistant tuberculosis, in Ecuador. International Journal of Tuberculosis and Lung Diseases, 14(10), 1316-1322.

Rørdam, H., 1911. De sidste 10 aars tuberkulosekampe i Danmark. Ugeskrift for læger, 73(15), 511-523.

Sakula, A., 1982. Robert Koch: Centenary of the discovery of the tubercle bacillus, 1882. Thorax, 37(4), 246-251.

Schelde Møller, E., 1950. Nationalforeningen til tuberkulosens bekæmpelse. Egmont H. Petersens Kgl. hof-bogtrykkeri, Copenhagen, Denmark.

Seligman, E.R.A., 1908. Progressive Taxation in Theory and Practice. American Economic Association Quarterly, 9(4), 1-334

Szreter, S., 1988. The Importance of Social Intervention in Britain's Mortality Decline c. 1850-1914: A Reinterpretation of the Role of Public Health. Social History of Medicine, 1(1), $1-37$.

Simonsen, K., 1947. Tuberkulose Problemer: Med Særligt Henblik på det Forebyggende Arbejde. Nyt Nordisk Forlag Arnold Busck, København 1947.

Smith, S., Jacobs, R.F., Wilson, C.B., 1997. Immunobiology of childhood tuberculosis: A window on the ontogeny of cellular immunity. Journal of Pediatrics, 131(1), 16-26. 
Thorton, R., 2008. The Demand for, and Impact of, Learning HIV Status," American Economic Review, 98(5), 1829-1863.

Tiemersma, E.W., van der Werf, M.J., Borgdoff, M.W., Williams, B.G., Nagelkerke, N.J.D., 2011. Natural history of tuberculosis: duration and fatality of untreated pulmonary tuberculosis in HIV negative patients: A systematic review. Plos One, 6(1), 1-13.

Vynnycky, E., Fine, P.E.M., 1997. The natural history of tuberculosis: the implications of age-dependent risks of disease and the role of reinfection. Epidemiology and Infection, 119, 183-201.

Weil, D.N., 2014. Health and Economic Growth. Durlauf, S.N., Aghion, P. (eds). Handbook of Economic Growth, North-Holland, Netherlands.

Williamson, D.J., 1910. The anti-tuberculosis dispensary movement. British Journal of Tuberculosis, 4(3), 157-168.

Wilson, G.S., 1943. The pasteurization of milk. British Medical Journal, 1(4286), 261-262.

Winge, K., 1952. Denmark's anti-tuberculosis campaign. Irish Journal of Medicial Science, $27(5), 218-226$.

World Health Organization, 2015. Global tuberculosis report 2015. World Health Organization, France.

Wüst, M., 2012. Early interventions and infant health: Evidence from the Danish home visiting program. Labour Economics, 19(4), 484-495.

\section{Statistics}

Danmarks Statistik, Statistiske Meddelelser, 1905, 1914, 1918, 1919, 1922.

Nationalforeningen til Tuberkulosens Bekæmpelse, 1914-1940. Beretning om virksomheden. Egmont H. Petersens Kgl. Hof-Bogtrykkeri, Copenhagen.

Sundhedsstyrelsen, Dødsaarsagerne i Kongeriget Danmarks byer, 1890-1919. 
Sundhedsstyrelsen, Dødsaarsagerne i Kongeriget Danmark, 1920-1950.

Sundhedsstyrelsen, Medicinalberetningen for Kongeriget Danmark, 1890-1950. 
Figure 1: TB mortality in the Danish cities, 1890-1950

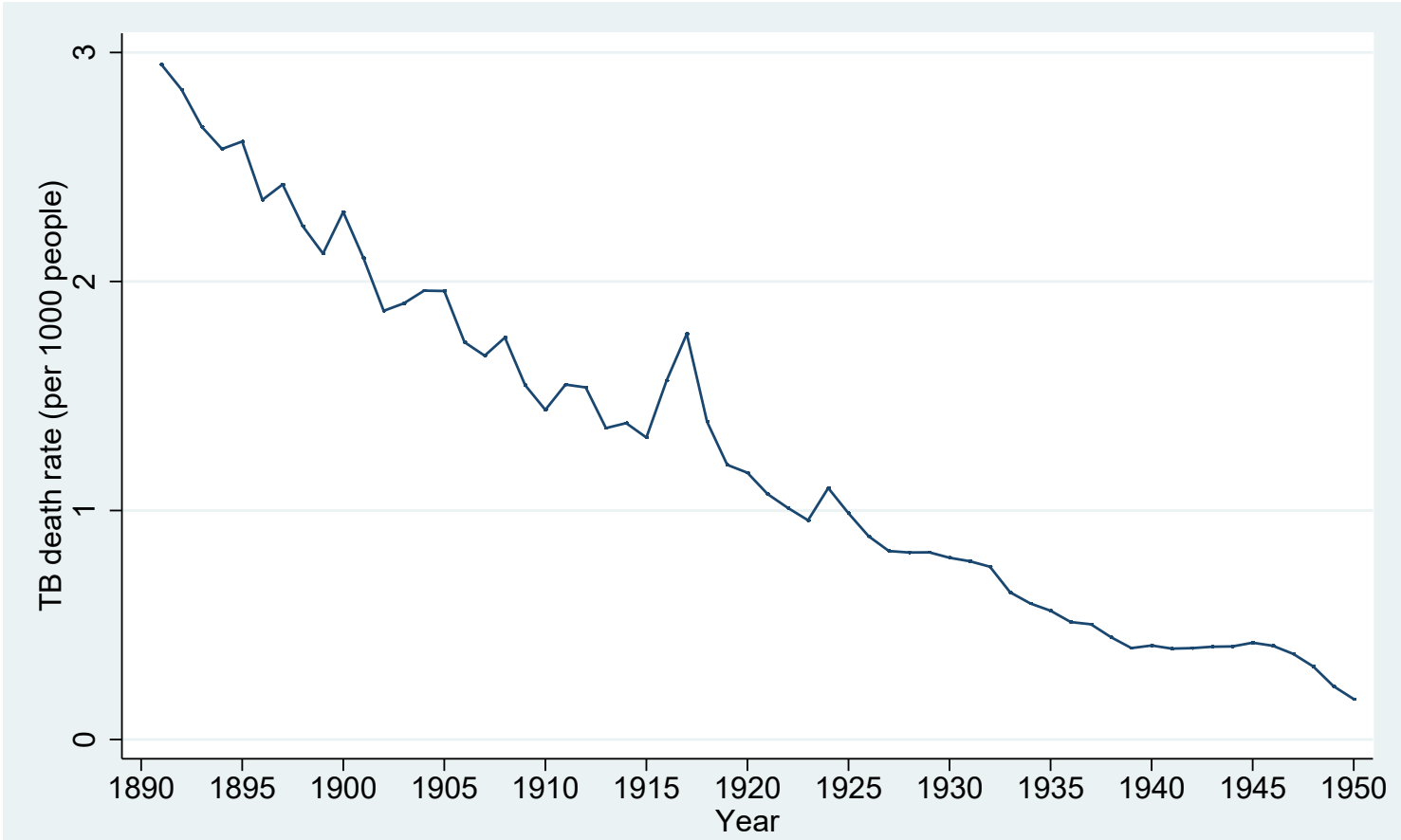

Notes: The graph shows the national development of TB mortality in the Danish cities per 1,000 people. Source: the Cause of Death Statistics (1890-1950) and the authors' own calculations. 
Figure 2: Spread of TB dispensaries across Danish cities
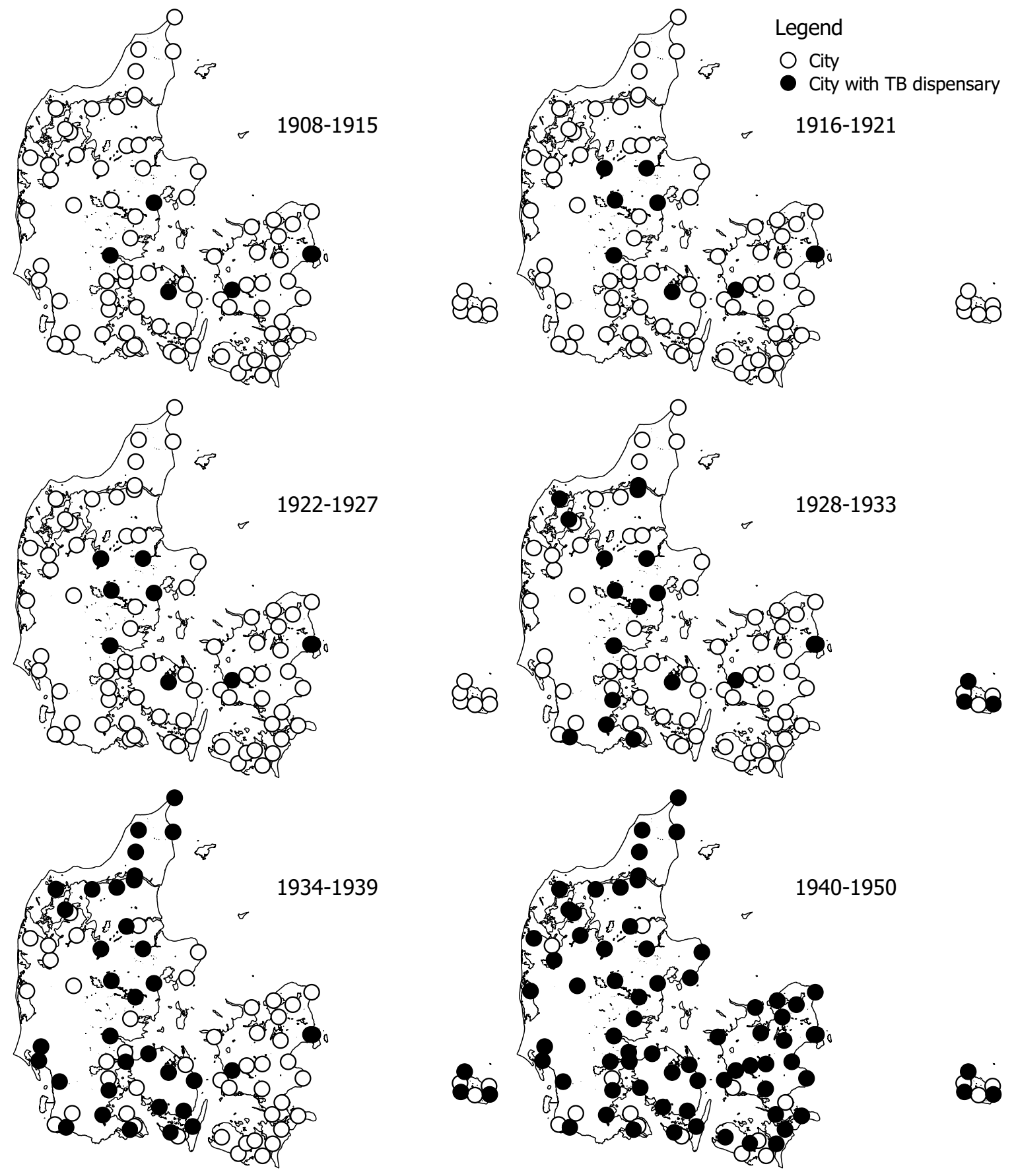

Notes: The maps show cities in the sample and when TB dispensaries were established. Source: Medical Reports (1890-1950). 
Figure 3: Average TB rate before and after TB dispensary

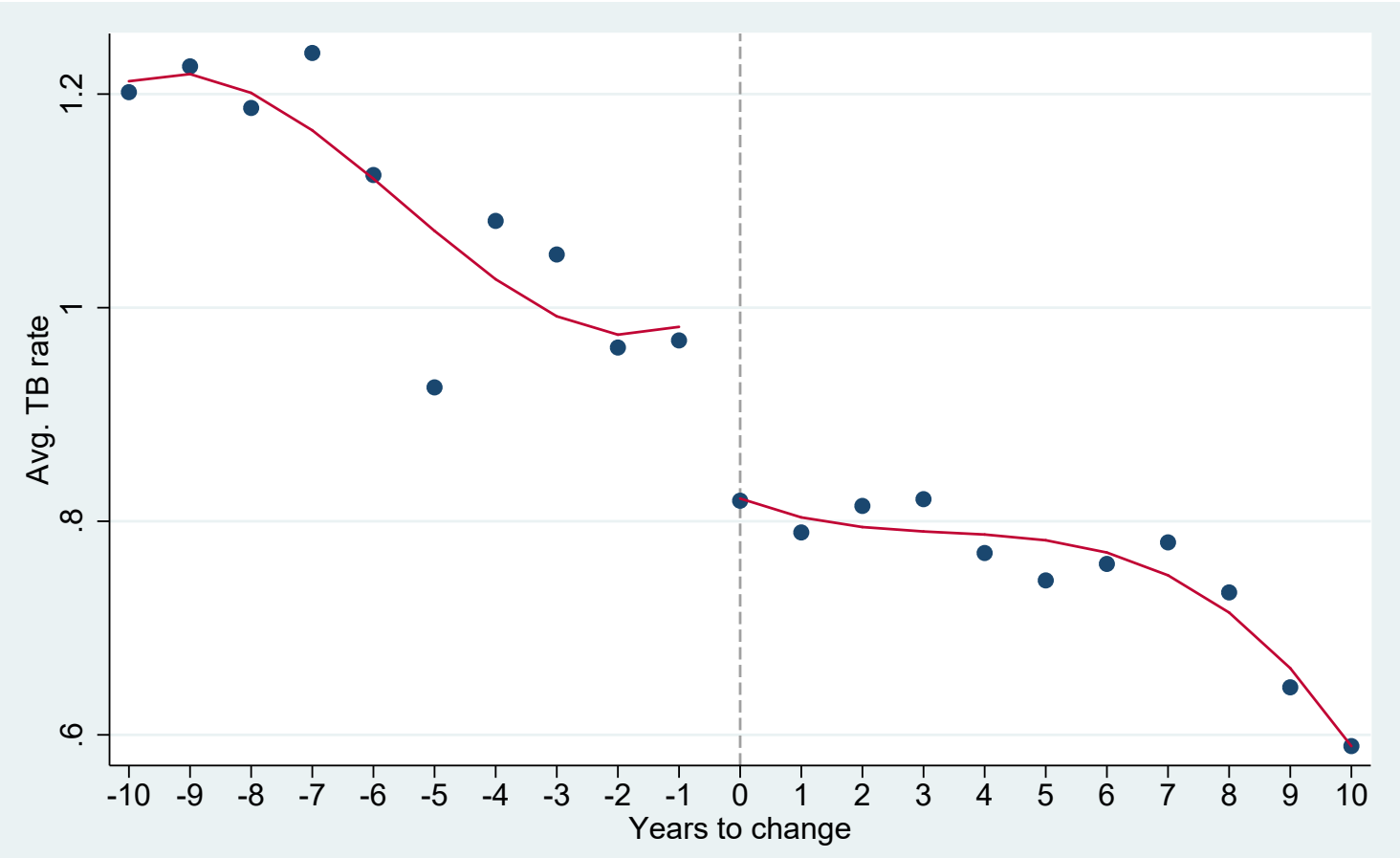

Notes: The graph shows the average TB rate before and after the introduction of a TB dispensary in a city, for the period 1890-1939. The red lines are fitted cubic polynomials before and after the introduction of a TB dispensary in a city. 
Figure 4: Average TB deaths 0 to 48 months before and after TB dispensary



Notes: The graph shows the average monthly TB deaths across rural and urban areas before and after a change in the number of TB dispensarais in the rural or urban areas weighted on the change in dispensaries, for the period 1920-1939. The red lines are fitted cubic polynomials before and after the change in the number of TB dispensaries. 
Figure 5: Simulation of the TB death rate

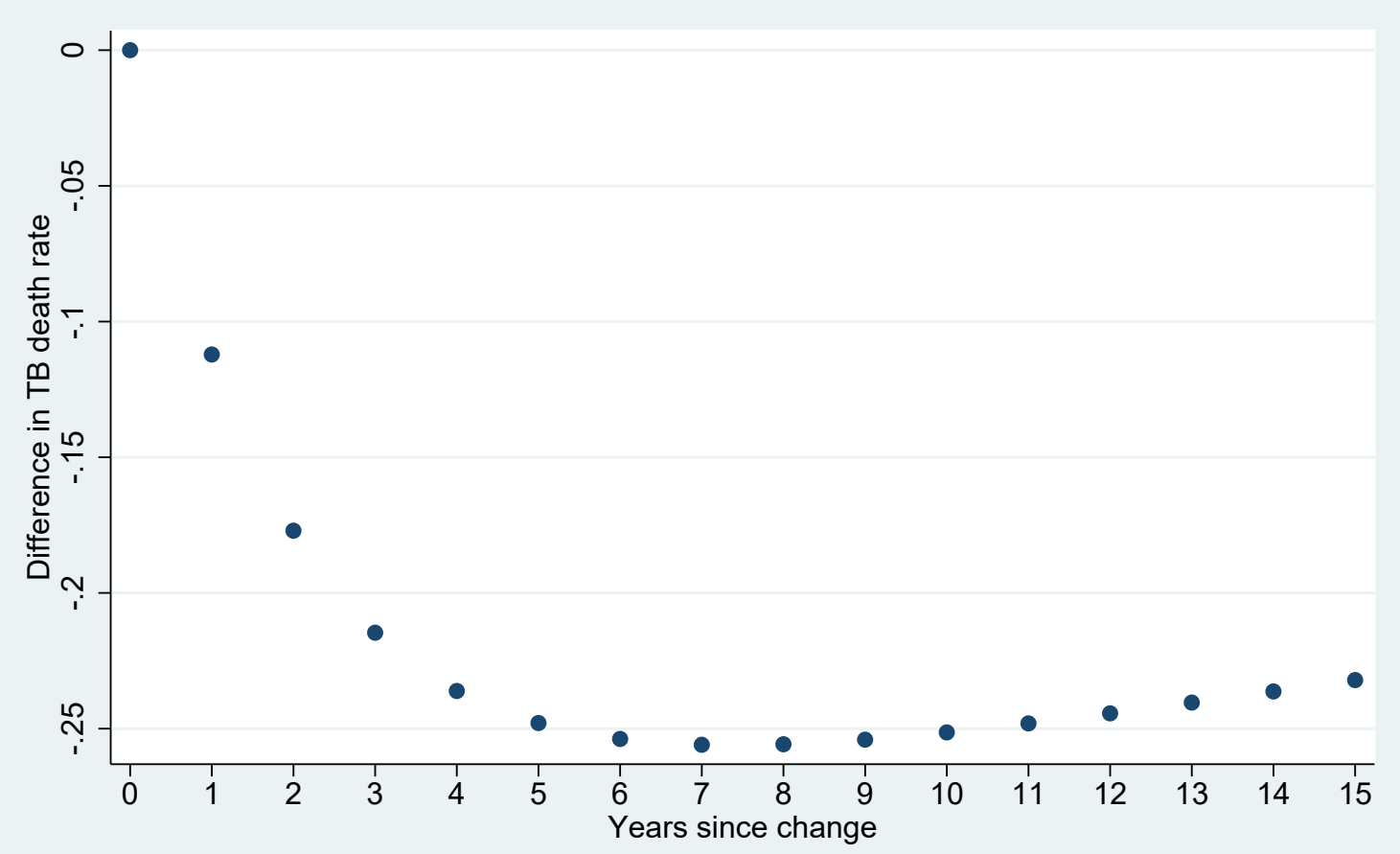

Notes: The graph shows the simulated differences bewteen the path of the TB death rate with dispensaries and the counterfactual in the SIR model. 
Figure 6: Event-study estimates of the impact on TB before and after TB dispensary

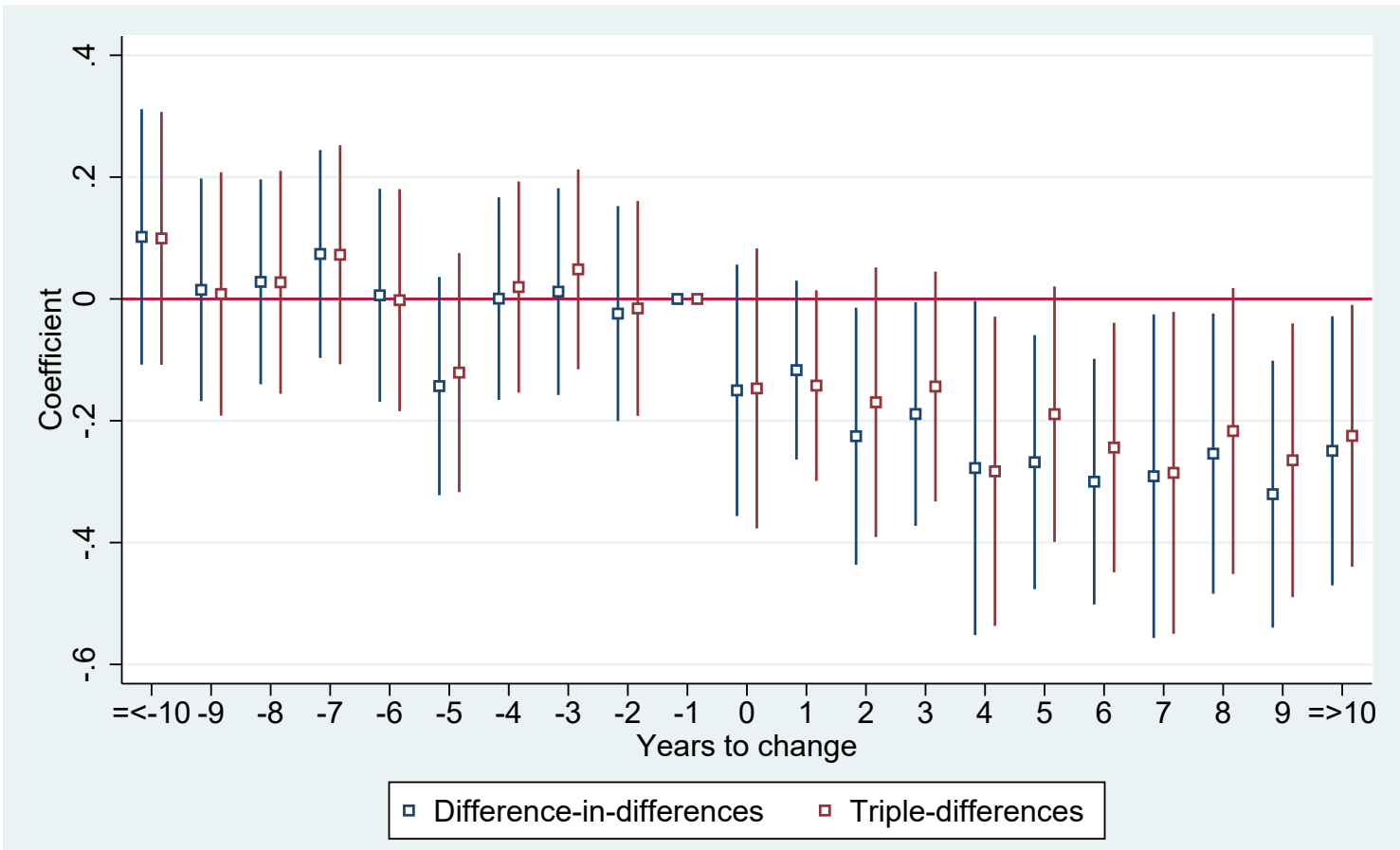

Notes: The graph shows the $\hat{\beta}_{j}$ coefficients and their 95 percent confidence interval from estimating equations (3) and (4) with year $\tau-1$ as the baseline, the year before the intervention. The estimated coefficients are shown in Table A2 in the Online Appendix. 
Figure 7: The distribution of coefficients from random placebo tests
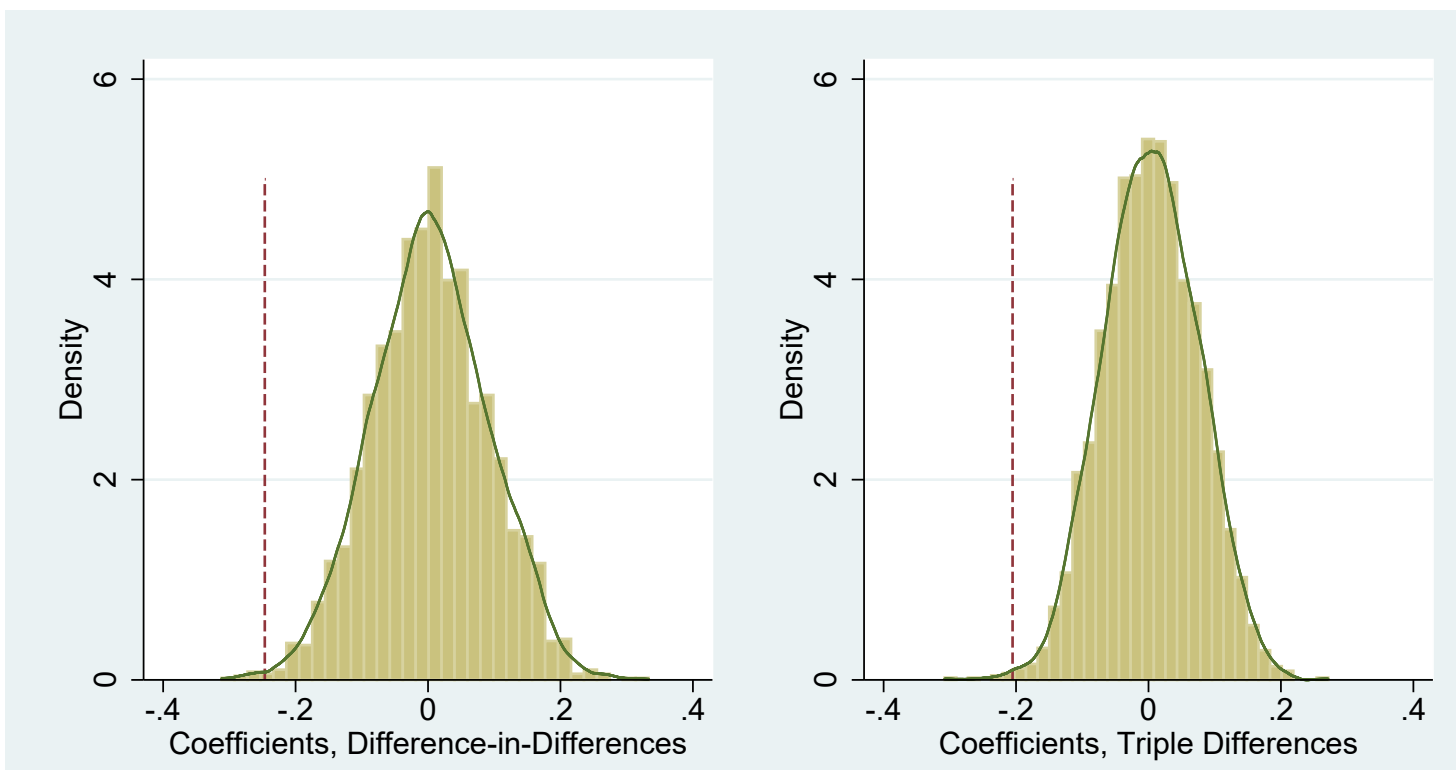

Notes: The left and right graphs show histograms of the distribution of the coefficient on TB dispensary obtained by re-estimating the difference-in-differences model in equation (1) and the triple differences model in equation (2) with the commissioning date of the TB dispensaries randomly reshuffled 2,499 time, along with kernel density plots. The stippled vertical lines show the placement of the coefficients from running the difference-in-differences and triple-differences model with the true commissioning date. The kernel density functions are estimated with the Epanechnikov kernel and a bandwidth $\sim 0.016$ and 0.014 for the difference-in-differences and the triple differences models, respectively. The areas to the left of the stippled lines are 0.005 and 0.004 for the difference-in-differences and the triple-differences models, respectively. 
Figure 8: TB deaths per month from 1920 to 1939 in urban and rural areas
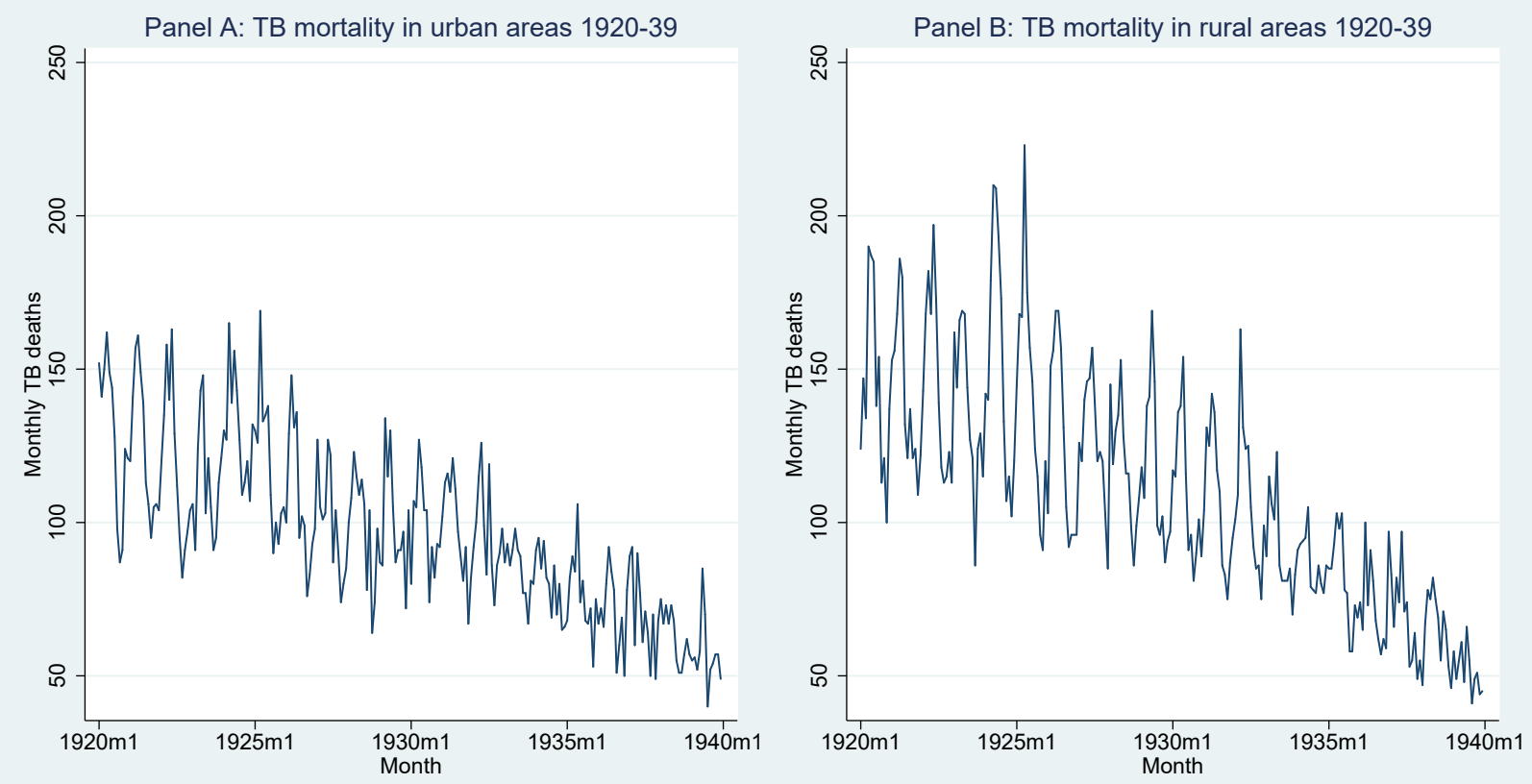

Notes: Panel A depicts the monthly development of TB mortality in urban areas from 1920 to 1939, and Panel B depicts the monthly development of TB mortality in rural areas from 1920 to 1939. Source: The Cause of Death Statistics (1920-1939). 
Figure 9: Event-study estimates of the impact on TB of the change in the number of TB dispensaries 0 to 48 months after the change

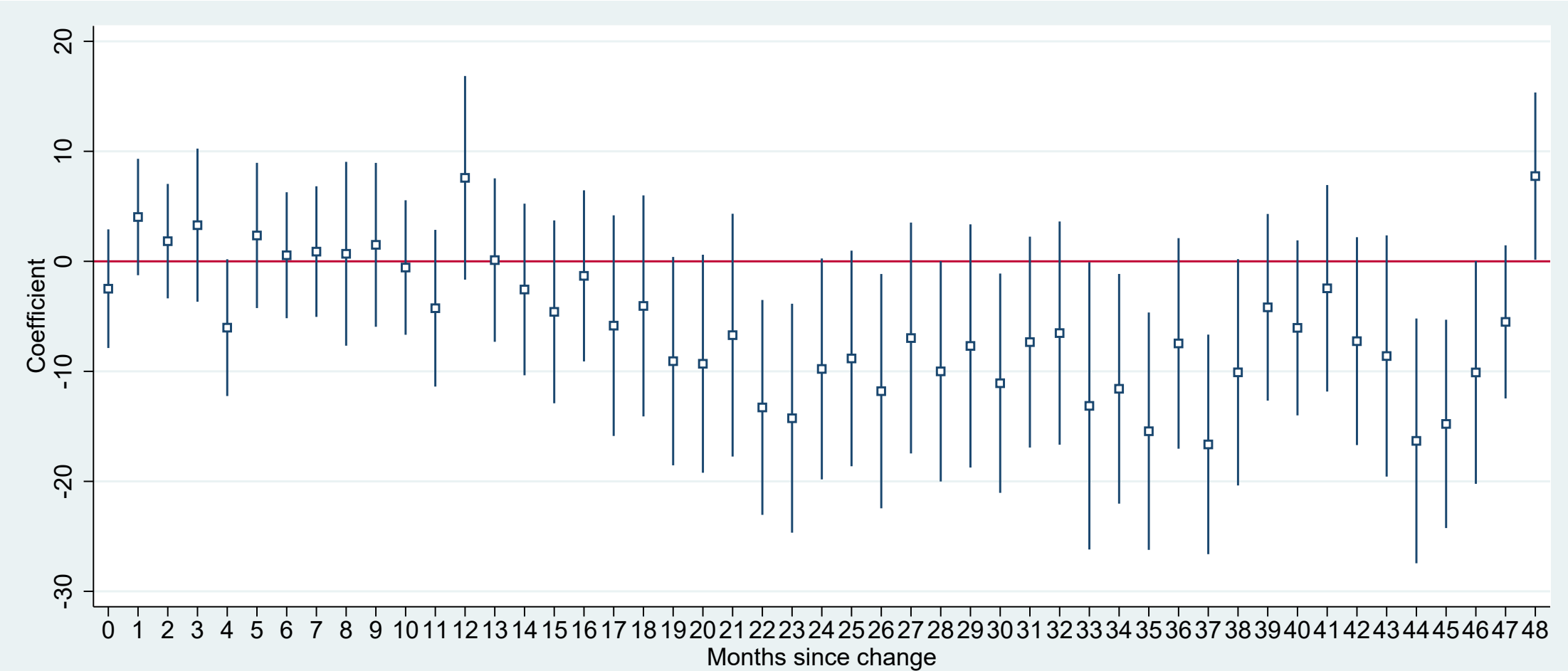

Notes: The graph shows the coefficients and the robust 95 percent confidence interval from regressing the stacked causes of death from TB, measles, scarlet fever, alcohol poisoning, accidents and homicides, suicides, diphtheria, typhoid fever, puerperal fever, pneumonia, and cancer, varying at the monthly level, and by rural and urban areas from January 1920 to December 1939, on 0 to 48 lags of the change in the stacked number of TB dispensaries in the rural or urban areas at time $t$ multiplied by an indicator equal to one if the disease on the left-hand-side is TB, controlling for disease, urban, month, year, disease-by-urban-by-month, disease-by-urban-by-year, disease-by-month-by-year, and urban-by-month-by-year fixed effects. 
Figure 10: Number of patients in sanatoria and TB hospitals and number of TB dispensaries
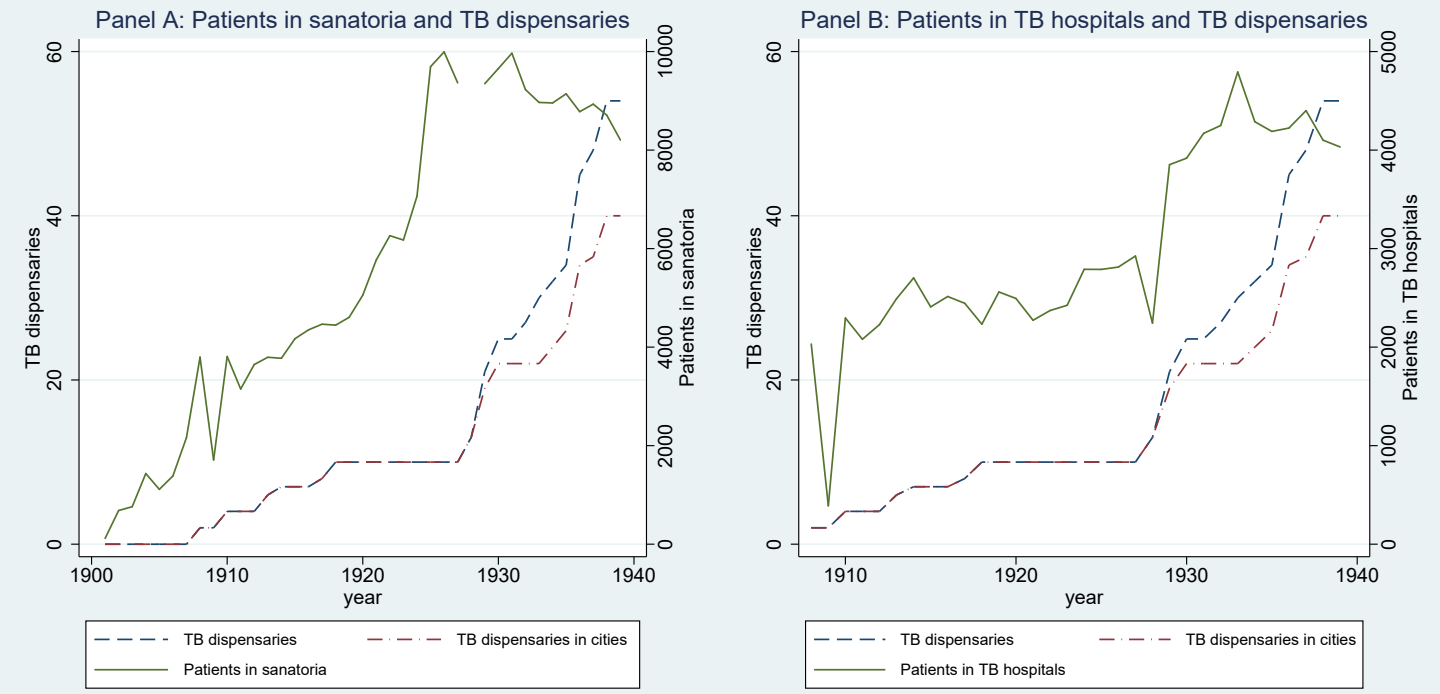

Notes: Panel A plots the number of sanatorium patients and the number of TB dispensaries in total and in the cities, and Panel B plots the number of TB hospital patients and the number of TB dispensaries in total and in the citites. The number of sanatorium patients in 1928 is unavailable. 
Table 1: Summary statistics

\begin{tabular}{lcccccc}
\hline \hline Variable & Period & No. Obs. & Mean & Std. Dev. & Min & Max \\
\hline TB rate & $1890-1939$ & 3,981 & 1.305 & 1.036 & 0.000 & 7.123 \\
Death rate & $1890-1939$ & 3,981 & 13.931 & 3.729 & 2.500 & 42.740 \\
TB rate & $1901-1939$ & 3,165 & 1.033 & 0.804 & 0.000 & 6.667 \\
Pulmonary rate & $1901-1939$ & 3,165 & 0.789 & 0.661 & 0.000 & 5.556 \\
Birth rate & $1901-1939$ & 3,165 & 22.348 & 5.766 & 1.429 & 45.455 \\
Child mortality rate & $1901-1939$ & 3,165 & 113.136 & 53.063 & 0.000 & 583.333 \\
Death rate & $1901-1939$ & 3,165 & 12.978 & 2.912 & 2.500 & 33.333 \\
Accident and suicide rate & $1901-1939$ & 3,165 & 0.472 & 0.396 & 0.000 & 3.077 \\
Cancer rate & $1901-1939$ & 3,165 & 1.421 & 0.746 & 0.000 & 13.333 \\
Diphtheria rate & $1901-1939$ & 3,165 & 0.081 & 0.202 & 0.000 & 2.210 \\
Influenza rate & $1901-1939$ & 3,165 & 0.347 & 0.699 & 0.000 & 9.630 \\
Pneumonia rate & $1901-1939$ & 3,165 & 1.250 & 0.755 & 0.000 & 6.667 \\
Polio rate & $1901-1939$ & 3,165 & 0.004 & 0.034 & 0.000 & 0.625 \\
Scarlet fever rate & $1901-1939$ & 3,165 & 0.026 & 0.123 & 0.000 & 3.268 \\
\hline
\end{tabular}

Notes: This table reports summary statistics for the main variables used in the regression analysis. See Table A1 in the Online Appendix for the definition of the variables. 
Table 2: Effect of TB dispensaries using city-by-year data on TB

\begin{tabular}{|c|c|c|c|c|c|}
\hline \multirow[t]{2}{*}{ Dep. variable: } & TB rate & $\log (\mathrm{TB}$ rate $)$ & TB rate & $\mathrm{TB}$ rate & Pulmonary rate \\
\hline & (1) & $(2)$ & $(3)$ & (4) & $(5)$ \\
\hline TB dispensary ${ }_{c, t}$ & $\begin{array}{c}-0.247^{* * *} \\
(0.076)\end{array}$ & $\begin{array}{c}-0.174^{* * *} \\
(0.053)\end{array}$ & $\begin{array}{c}-0.213^{* * *} \\
(0.065)\end{array}$ & $\begin{array}{c}-0.166^{* * *} \\
(0.053)\end{array}$ & $\begin{array}{c}-0.177^{* * *} \\
(0.056)\end{array}$ \\
\hline $\mathrm{TB}$ rate $_{c, t-1}$ & & & $\begin{array}{c}0.148^{* * *} \\
(0.024)\end{array}$ & $\begin{array}{c}0.118^{* * *} \\
(0.023)\end{array}$ & \\
\hline TB rate $_{c, t-2}$ & & & & $\begin{array}{l}0.041^{* *} \\
(0.020)\end{array}$ & \\
\hline TB rate $_{c, t-3}$ & & & & $\begin{array}{c}0.104^{* * *} \\
(0.025)\end{array}$ & \\
\hline $\mathrm{TB}$ rate $_{c, t-4}$ & & & & $\begin{array}{l}0.055^{* *} \\
(0.022)\end{array}$ & \\
\hline TB rate $_{c, t-5}$ & & & & $\begin{array}{c}0.027 \\
(0.020)\end{array}$ & \\
\hline Avg. dep. var. & 1.306 & 0.101 & 1.280 & 1.171 & 0.789 \\
\hline Long run effect & & & $-0.250^{* * *}$ & $-0.253^{* * *}$ & \\
\hline City FE & Yes & Yes & Yes & Yes & Yes \\
\hline Year FE & Yes & Yes & Yes & Yes & Yes \\
\hline Time period & 1890-1939 & 1890-1939 & 1891-1939 & 1895-1939 & 1901-1939 \\
\hline Observations & 3,981 & 3,662 & 3,894 & 3,546 & 3,165 \\
\hline R-squared & 0.53 & 0.58 & 0.54 & 0.52 & 0.39 \\
\hline Cities & 87 & 87 & 87 & 87 & 87 \\
\hline
\end{tabular}

Notes: The table reports least squares estimates. In columns (1), (3), and (4) the left-hand-side variable is the TB mortality per 1,000 people, in column (2) the log is taken of this variable, and in column (5) the left-hand-side variable is the pulmonary TB mortality per 1,000 people. All regressions include city and year fixed effects. TB dispensary ${ }_{c, t}$ is an indicator variable equal to one after the introduction of a TB dispensary, and TB rate ch,t-i $_{i}$ is the $i$ year lagged dependent variable. Long run effect is the steady-state value of the estimated model in columns (3), and (4). Robust standard errors clustered at the city level are in parentheses.

${ }^{*},{ }^{* *}$ and ${ }^{* * *}$ determine significance levels of 10 percent, 5 percent and 1 percent, respectively. 
Table 3: Placebo outcome regressions

\begin{tabular}{|c|c|c|c|c|c|c|c|c|}
\hline \multirow{3}{*}{ Dep. variable: } & \multirow{2}{*}{$\begin{array}{c}\text { Baseline } \\
\mathrm{TB} \\
\text { rate }\end{array}$} & \multicolumn{7}{|c|}{ Placebo } \\
\hline & & $\begin{array}{l}\text { Cancer } \\
\text { rate }\end{array}$ & $\begin{array}{l}\text { Influen- } \\
\text { za rate }\end{array}$ & $\begin{array}{l}\text { Pneumo- } \\
\text { nia rate }\end{array}$ & $\begin{array}{c}\text { Accident and } \\
\text { suicide rate }\end{array}$ & $\begin{array}{c}\text { Scarlet } \\
\text { fever rate }\end{array}$ & $\begin{array}{l}\text { Diphthe- } \\
\text { ria rate }\end{array}$ & $\begin{array}{l}\text { Polio } \\
\text { rate }\end{array}$ \\
\hline & (1) & $(2)$ & $(3)$ & (4) & $(5)$ & (6) & (7) & (8) \\
\hline TB dispensary dit & $\begin{array}{c}-0.223^{* * *} \\
(0.066)\end{array}$ & $\begin{array}{l}-0.035 \\
(0.051)\end{array}$ & $\begin{array}{l}-0.017 \\
(0.026)\end{array}$ & $\begin{array}{l}-0.064 \\
(0.057)\end{array}$ & $\begin{array}{l}-0.008 \\
(0.025)\end{array}$ & $\begin{array}{l}-0.010 \\
(0.006)\end{array}$ & $\begin{array}{c}0.011 \\
(0.015)\end{array}$ & $\begin{array}{l}-0.003 \\
(0.002)\end{array}$ \\
\hline Avg. dep. var. & 1.034 & 1.421 & 0.347 & 1.250 & 0.472 & 0.026 & 0.081 & 0.005 \\
\hline City FE & Yes & Yes & Yes & Yes & Yes & Yes & Yes & Yes \\
\hline Year FE & Yes & Yes & Yes & Yes & Yes & Yes & Yes & Yes \\
\hline Time period & 1901-1939 & 1901-1939 & 1901-1939 & 1901-1939 & 1901-1939 & 1901-1939 & 1901-1939 & 1901-1939 \\
\hline Observations & 3,165 & 3,165 & 3,165 & 3,165 & 3,165 & 3,165 & 3,165 & 3,165 \\
\hline R-squared & 0.44 & 0.15 & 0.60 & 0.19 & 0.10 & 0.09 & 0.11 & 0.09 \\
\hline Cities & 87 & 87 & 87 & 87 & 87 & 87 & 87 & 87 \\
\hline
\end{tabular}

Notes: The table reports least squares estimates. In column (1) the left-hand-side variable is the TB mortality per 1,000 people, in column (2) it is the cancer mortality per 1,000 people, in column (3) it is the influenza mortality per 1,000 people, in column (4) it is the pneumonia mortality per 1,000 people, in column (5) it is accident and suicide deaths per 1,000 people, and from 1931 including homicides, in column (6) it is the scarlet fever mortality per 1,000 people, in column (7) it is the diphtheria mortality per 1,000 people, and in column (8) it is the polio mortality per 1,000 people. All regressions include city and year fixed effects. TB dispensary ${ }_{c, t}$ is an indicator variable equal to one after the introduction of a TB dispensary. Robust standard errors clustered at the city level are in parentheses.

${ }^{*},{ }^{* *}$ and ${ }^{* * *}$ determine significance levels of 10 percent, 5 percent and 1 percent, respectively. 
Table 4: Effect of TB dispensaries using city-by-year data on other outcomes

\begin{tabular}{|c|c|c|c|c|c|c|}
\hline \multirow[t]{2}{*}{ Dep. variable: } & $\begin{array}{l}\text { Child mor- } \\
\text { tality rate }\end{array}$ & $\begin{array}{l}\log (\text { Child mor- } \\
\text { tality rate })\end{array}$ & Death rate & $\begin{array}{l}\log (\text { Death } \\
\text { rate })\end{array}$ & Birth rate & $\begin{array}{c}\log (\text { Birth } \\
\text { rate })\end{array}$ \\
\hline & $(1)$ & $(2)$ & $(3)$ & $(4)$ & $(5)$ & $(6)$ \\
\hline TB dispensary ${ }_{c, t}$ & $\begin{array}{c}-13.204^{* * *} \\
(3.980)\end{array}$ & $\begin{array}{c}-0.109^{* * *} \\
(0.038)\end{array}$ & $\begin{array}{c}-0.605^{* *} \\
(0.279)\end{array}$ & $\begin{array}{c}-0.044^{* *} \\
(0.021)\end{array}$ & $\begin{array}{c}0.075 \\
(0.512)\end{array}$ & $\begin{array}{c}0.017 \\
(0.026)\end{array}$ \\
\hline Avg. dep. var. & 113.136 & 4.653 & 13.931 & 2.601 & 22.348 & 3.071 \\
\hline City FE & Yes & Yes & Yes & Yes & Yes & Yes \\
\hline Year FE & Yes & Yes & Yes & Yes & Yes & Yes \\
\hline Time period & 1901-1939 & 1901-1939 & 1890-1939 & 1890-1939 & 1901-1939 & 1901-1939 \\
\hline Observations & 3,165 & 3,089 & 3,981 & 3,981 & 3,165 & 3,165 \\
\hline R-squared & 0.30 & 0.30 & 0.49 & 0.47 & 0.67 & 0.65 \\
\hline Cities & 87 & 87 & 87 & 87 & 87 & 87 \\
\hline
\end{tabular}

Notes: The table reports least squares estimates. In column (1) the left-hand-side variable is the mortality among 0 - to 5 -year-olds per 1,000 live births from 1901 to 1936, and from 1937 to 1939 the mortality among 0- to 4-year-olds per 1,000 live births, in column (2) the log is taken of this variable, in column (3) the left-hand-side variable is the mortality excluding stillborns per 1,000 people, in column (4) the log is taken of this variable, in column (5) the left-hand-side variable is the number of births excluding stillborns per 1,000 people, and in column (6) the log is taken of this variable. All regressions include city and year fixed effects. TB dispensary ${ }_{c, t}$ is an indicator variable equal to one after the introduction of a TB dispensary. Robust standard errors clustered at the city level are in parentheses.

${ }^{*},{ }^{* *}$ and ${ }^{* * *}$ determine significance levels of 10 percent, 5 percent and 1 percent, respectively. 
Table 5: Effect of TB dispensaries using city-by-year-by-disease data

\begin{tabular}{|c|c|c|c|}
\hline \multirow[t]{2}{*}{ Dep. variable: } & \multicolumn{3}{|c|}{ Disease } \\
\hline & $(1)$ & $(2)$ & $(3)$ \\
\hline TB dispensary ${ }_{c, t}$ & $\begin{array}{c}-0.205^{* * *} \\
(0.064)\end{array}$ & $\begin{array}{c}-0.194^{* * *} \\
(0.062)\end{array}$ & $\begin{array}{c}-0.200^{* * *} \\
(0.061)\end{array}$ \\
\hline Disease $_{c, t-1}$ & & $\begin{array}{c}0.022 \\
(0.017)\end{array}$ & $\begin{array}{c}0.011 \\
(0.017)\end{array}$ \\
\hline Disease $_{c, t-2}$ & & & $\begin{array}{c}0.044^{* * *} \\
(0.014)\end{array}$ \\
\hline Disease $_{c, t-3}$ & & & $\begin{array}{c}0.014 \\
(0.014)\end{array}$ \\
\hline Disease $_{c, t-4}$ & & & $\begin{array}{c}0.013 \\
(0.012)\end{array}$ \\
\hline Disease $_{c, t-5}$ & & & $\begin{array}{l}-0.008 \\
(0.012)\end{array}$ \\
\hline Avg. TB rate & 1.034 & 1.013 & 0.923 \\
\hline Long run effect & & $-0.199^{* * *}$ & $-0.216^{* * *}$ \\
\hline City FE & Yes & Yes & Yes \\
\hline Year FE & Yes & Yes & Yes \\
\hline Disease FE & Yes & Yes & Yes \\
\hline Disease FE $\times$ Year FE & Yes & Yes & Yes \\
\hline Disease FE $\times$ City FE & Yes & Yes & Yes \\
\hline City FE $\times$ Year FE & Yes & Yes & Yes \\
\hline Time period & 1901-1939 & $1902-1939$ & $1906-1939$ \\
\hline Observations & 25,320 & 24,624 & 21,840 \\
\hline R-squared & 0.70 & 0.70 & 0.71 \\
\hline Cities & 87 & 87 & 87 \\
\hline
\end{tabular}

Notes: The table reports least squares estimates. In columns (1) to (3) the left-hand-side variable is the stacked causes of death from TB, cancer, influenza, pneumonia, accidents and suicides (including homicides from 1931), scarlet fever, diphtheria, and polio per 1,000 people. All regressions include city, year, disease, disease-by-year, disease-by-city, and city-by-year fixed effects. TB dispensary ${ }_{c, t}$ is an indicator variable equal to one after the introduction of a TB dispensary multiplied by an indicator equal to one if the disease on the left-hand side is $\mathrm{TB}$, and Disease ${ }_{c, t-i}$ is the $i$ year lagged dependent variable. Robust standard errors clustered at the city level are in parentheses.

${ }^{*},{ }^{* *}$ and ${ }^{* * *}$ determine significance levels of 10 percent, 5 percent and 1 percent, respectively. 
Table 6: Effect of TB dispensaries controlling for neighboring dispensaries

\begin{tabular}{|c|c|c|c|c|c|c|}
\hline \multirow[t]{2}{*}{ Dep. variable: } & $\mathrm{TB}$ rate & Disease & $\mathrm{TB}$ rate & Disease & $\mathrm{TB}$ rate & Disease \\
\hline & $(1)$ & $(2)$ & $(3)$ & $(4)$ & $(5)$ & $(6)$ \\
\hline TB dispensary ditt & $\begin{array}{c}-0.240^{* * *} \\
(0.076)\end{array}$ & $\begin{array}{c}-0.203^{* * *} \\
(0.065)\end{array}$ & $\begin{array}{c}-0.275^{* * *} \\
(0.082)\end{array}$ & $\begin{array}{c}-0.249^{* * *} \\
(0.068)\end{array}$ & $\begin{array}{c}-0.266^{* * *} \\
(0.091)\end{array}$ & $\begin{array}{c}-0.250^{* * *} \\
(0.075)\end{array}$ \\
\hline TB dispensary ${ }_{c, t, 10}$ & $\begin{array}{c}0.099 \\
(0.197)\end{array}$ & $\begin{array}{c}0.036 \\
(0.171)\end{array}$ & & & & \\
\hline TB dispensary ${ }_{c, t, 30}$ & & & $\begin{array}{l}-0.081 \\
(0.090)\end{array}$ & $\begin{array}{l}-0.123 \\
(0.077)\end{array}$ & & \\
\hline TB dispensary ${ }_{c, t, 50}$ & & & & & $\begin{array}{l}-0.036 \\
(0.080)\end{array}$ & $\begin{array}{l}-0.086 \\
(0.065)\end{array}$ \\
\hline Avg. TB rate & 1.306 & 1.034 & 1.306 & 1.034 & 1.306 & 1.034 \\
\hline City FE & Yes & Yes & Yes & Yes & Yes & Yes \\
\hline Year FE & Yes & Yes & Yes & Yes & Yes & Yes \\
\hline Disease FE & No & Yes & No & Yes & No & Yes \\
\hline Disease $\mathrm{FE} \times$ Year FE & No & Yes & No & Yes & No & Yes \\
\hline Disease FE $\times$ City FE & No & Yes & No & Yes & No & Yes \\
\hline City FE $\times$ Year FE & No & Yes & No & Yes & No & Yes \\
\hline Time period & $1890-1939$ & 1901-1939 & $1890-1939$ & 1901-1939 & $1890-1939$ & 1901-1939 \\
\hline Observations & 3,981 & 25,320 & 3,981 & 25,320 & 3,981 & 25,320 \\
\hline R-squared & 0.5333 & 0.6969 & 0.5341 & 0.6971 & 0.5335 & 0.6970 \\
\hline Cities & 87 & 87 & 87 & 87 & 87 & 87 \\
\hline
\end{tabular}

Notes: The table reports least squares estimates. In columns (1), (3), and (5) the left-hand-side variable is the TB mortality per 1,000 people, and in columns (2), (4), and (6) the left-hand-side variable is the stacked causes of death from TB, cancer, influenza, pneumonia, accidents and suicides (including homicides from 1931), scarlet fever, diphtheria, and polio per 1,000 people. All regressions include city and year fixed effects, and addtionally columns (2), (4), and (6) include disease, disease-by-year, disease-by-city, and city-by-year fixed effects. TB dispensary ${ }_{c, t}$ is an indicator variable equal to one after the introduction of a TB dispensary, and TB dispensary ${ }_{c, t, r}$ is an indicator variable equal to one after the introduction of a TB dispensary in a city within a $r$ kilometer radius unless TB dispensary ${ }_{c, t}=1$, multiplied by an indicator equal to one if the disease on the left-hand side is TB, respectively. Robust standard errors clustered at the city level are in parentheses. ${ }^{*},{ }^{* *}$ and ${ }^{* * *}$ determine significance levels of 10 percent, 5 percent and 1 percent, respectively. 
Table 7: Effect of TB dispensaries controlling for commissioning of TB hospitals and sanatoria

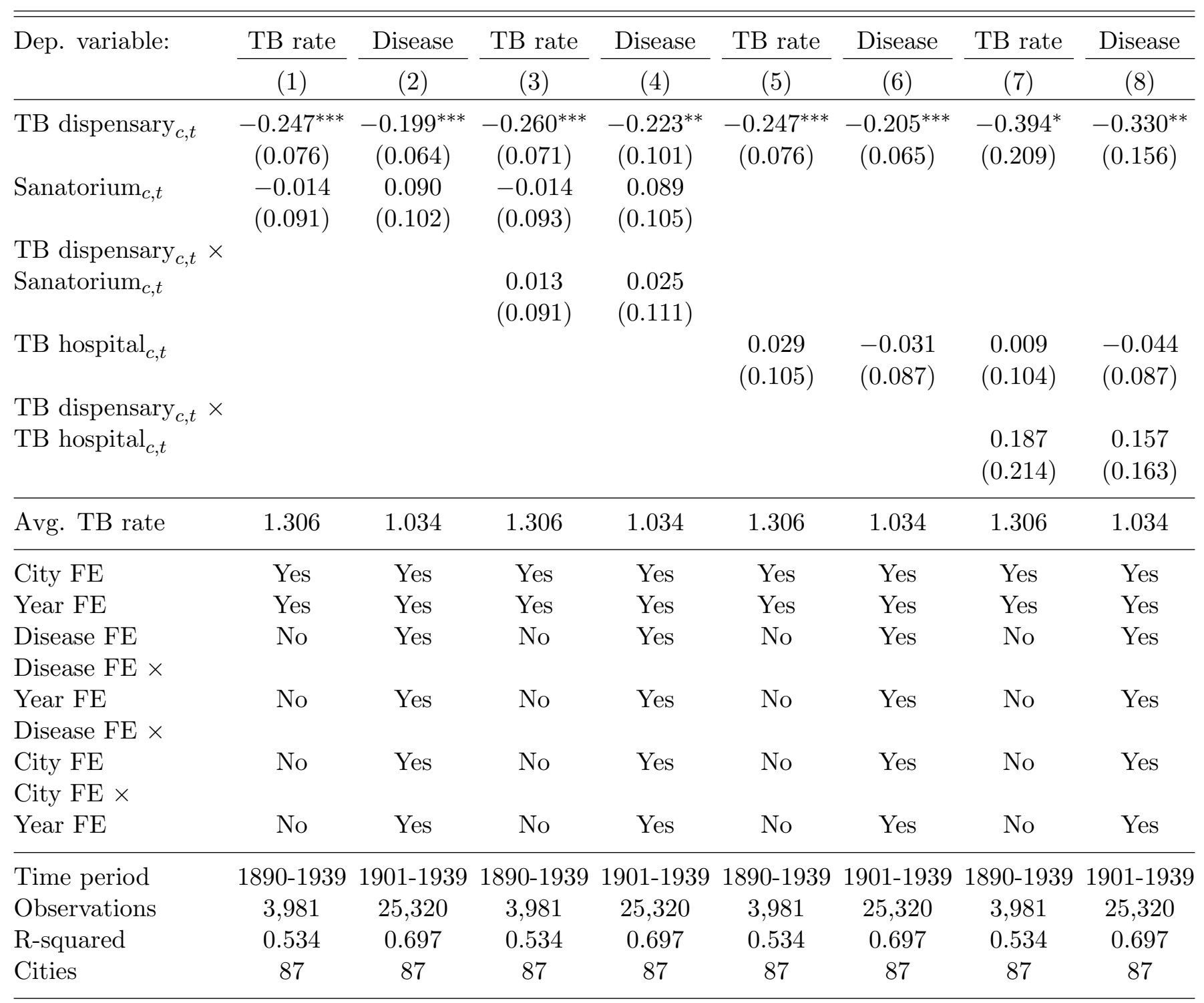

Notes: The table reports least squares estimates. In columns (1), (3), (5), and (7) the left-hand-side variable is the TB mortality per 1,000 people, in columns (2), (4), (6), and (8) the left-hand-side variable is the stacked causes of death from TB, cancer, influenza, pneumonia, accidents and suicides (including homicides from 1931), scarlet fever, diphtheria, and polio per 1,000 people. All regressions include city and year fixed effects, and addtionally columns (2), (4), (6), and (8) include disease, disease-by-year, disease-by-city, and city-by-year fixed effects. TB dispensary ${ }_{c, t}$ is an indicator variable equal to one after the introduction of a TB dispensary, TB hospital ${ }_{c, t}$ is an indicator variable equal to one after the introduction of a TB hospital within a county, Sanatorium ${ }_{c, t}$ is an indicator variable equal to one after the introduction of a sanatorium within $50 \mathrm{~km}$, and TB dispensary ${ }_{c, t} \times \mathrm{TB}$ hospital ${ }_{c, t}$ and TB dispensary ${ }_{c, t} \times$ Sanatorium $_{c, t}$ are interactions of the aforementioned variables. All right-hand-side variables are multiplied by an indicator equal to one if the disease on the left-hand side is TB. Robust standard errors clustered at the city level are in parentheses.

${ }^{*},{ }^{* *}$ and ${ }^{* * *}$ determine significance levels of 10 percent, 5 percent and 1 percent, respectively. 
Table 8: Effect of TB dispensaries controlling for pre-intervention income, taxpayers per capita, and inequality

\begin{tabular}{|c|c|c|c|c|c|c|c|c|}
\hline \multirow[t]{3}{*}{ Dep. variable: } & TB rate & Disease & $\mathrm{TB}$ rate & Disease & TB rate & Disease & TB rate & Disease \\
\hline & \multicolumn{4}{|c|}{ Baseline panel } & \multicolumn{4}{|c|}{ Excluding pre 1920 adopters } \\
\hline & $(1)$ & $(2)$ & $(3)$ & $(4)$ & $(5)$ & $(6)$ & $(7)$ & $(8)$ \\
\hline TB dispensary ${ }_{c, t}$ & $\begin{array}{c}-0.260^{* * *} \\
(0.074)\end{array}$ & $\begin{array}{c}-0.220^{* * *} \\
(0.061)\end{array}$ & $\begin{array}{c}-0.259^{* * *} \\
(0.071)\end{array}$ & $\begin{array}{c}-0.217^{* * *} \\
(0.057)\end{array}$ & $\begin{array}{c}-0.294^{* * *} \\
(0.098)\end{array}$ & $\begin{array}{c}-0.252^{* * *} \\
(0.080)\end{array}$ & $\begin{array}{c}-0.284^{* *} \\
(0.108)\end{array}$ & $\begin{array}{c}-0.245^{* * *} \\
(0.083)\end{array}$ \\
\hline Avg. TB rate & 1.306 & 1.034 & 1.309 & 1.034 & 1.309 & 1.034 & 1.309 & 1.034 \\
\hline Pre-int. $\log ($ income $) \times$ Year Fe & Yes & Yes & No & No & No & No & Yes & Yes \\
\hline Pre-int. income $\times$ Year Fe & No & No & Yes & Yes & No & No & Yes & Yes \\
\hline Pre-int. Gini × Year Fe & No & No & No & No & Yes & Yes & Yes & Yes \\
\hline City FE & Yes & Yes & Yes & Yes & Yes & Yes & Yes & Yes \\
\hline Year FE & Yes & Yes & Yes & Yes & Yes & Yes & Yes & Yes \\
\hline Disease FE & No & Yes & No & Yes & No & Yes & No & Yes \\
\hline Disease $\mathrm{FE} \times$ Year FE & No & Yes & No & Yes & No & Yes & No & Yes \\
\hline Disease FE $\times$ City FE & No & Yes & No & Yes & No & Yes & No & Yes \\
\hline City FE $\times$ Year FE & No & Yes & No & Yes & No & Yes & No & Yes \\
\hline Time period & 1890-1939 & 1901-1939 & 1890-1939 & 1901-1939 & 1890-1939 & 1901-1939 & $1890-1939$ & 1901-1939 \\
\hline Observations & 3,981 & 25,320 & 3,981 & 25,320 & 3,341 & 20,992 & 3,341 & 20,992 \\
\hline R-squared & 0.541 & 0.698 & 0.542 & 0.698 & 0.512 & 0.692 & 0.530 & 0.695 \\
\hline Cities & 87 & 87 & 87 & 87 & 68 & 68 & 68 & 68 \\
\hline
\end{tabular}

Notes: The table reports least squares estimates. In columns (1), (3), (5), and (7) the left-hand-side variable is the TB mortality per 1,000 people, and in columns (2), (4), (6), and (8) the left-hand-side variable is the stacked causes of death from TB, cancer, influenza, pneumonia, accidents and suicides (including homicides from 1931), scarlet fever, diphtheria, and polio per 1,000 people. All regressions include city and year fixed effects, and addtionally columns $(2),(4),(6)$, and (8) include disease, disease-by-year, disease-by-city, and city-by-year fixed effects. TB dispensary ${ }_{c, t}$ is an indicator variable equal to one after the introduction of a TB dispensary. In columns (1), (2), (7), and (8) the 1904-05 log income per capita interacted with year fixed effects is included, in columns (3), (4), (7), and (8) the 1904-05 taxpayers per capita is included, and in columns (5), (6), (7), and (8) the 1918-19

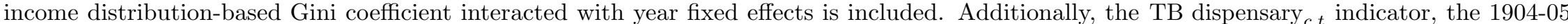
income, and the 1918-19 Gini coefficient are interacted with an indicator equal to one if the disease on the left-hand side is TB. For cities included in the data set later than 1904 and 1918, we use income per capita, taxpayers per capita, and Gini coefficients in the year they are added to the data. In columns (5), (6), (7), and (8) cities that adopted a TB dispensary before 1920 are excluded from the sample. Robust standard errors clustered at the city level are in parentheses.

${ }^{*},{ }^{* *}$ and ${ }^{* * *}$ determine significance levels of 10 percent, 5 percent and 1 percent, respectively. 
Table 9: Treatment heterogeneity of TB dispensaries

\begin{tabular}{|c|c|c|c|c|c|c|c|c|}
\hline \multirow[t]{3}{*}{ Dep. variable: } & TB rate & Disease & TB rate & Disease & TB rate & Disease & TB rate & Disease \\
\hline & \multicolumn{6}{|c|}{ Baseline panel } & \multicolumn{2}{|c|}{ Excluding pre 1920 adopters } \\
\hline & $(1)$ & $(2)$ & $(3)$ & $(4)$ & $(5)$ & $(6)$ & $(7)$ & $(8)$ \\
\hline TB dispensary ${ }_{c, t}$ & $\begin{array}{c}-0.182^{* * *} \\
(0.068)\end{array}$ & $\begin{array}{c}-0.171^{* * *} \\
(0.061)\end{array}$ & $\begin{array}{c}-0.237^{* * *} \\
(0.079)\end{array}$ & $\begin{array}{c}-0.204^{* * *} \\
(0.064)\end{array}$ & $\begin{array}{c}-0.219^{* * *} \\
(0.075)\end{array}$ & $\begin{array}{c}-0.189^{* * *} \\
(0.064)\end{array}$ & $\begin{array}{c}-0.313^{* * *} \\
(0.100)\end{array}$ & $\begin{array}{c}-0.272^{* * *} \\
(0.081)\end{array}$ \\
\hline $\begin{array}{l}\text { TB dispensary } \\
\text { Initial TB } \text { rate }_{c} \times \\
\end{array}$ & $\begin{array}{c}-0.161^{* *} \\
(0.076)\end{array}$ & $\begin{array}{c}-0.115^{*} \\
(0.059)\end{array}$ & & & & & & \\
\hline $\begin{array}{l}\text { TB dispensary } \\
\text { Pre-int. } \\
\text { pop. density }\end{array}$ & & & $\begin{array}{l}0.042^{*} \\
(0.023)\end{array}$ & $\begin{array}{l}0.062^{* *} \\
(0.028)\end{array}$ & & & & \\
\hline $\begin{array}{l}\text { TB dispensary } \\
\text { Pre-int. } \log (\text { income })_{c}\end{array}$ & & & & & $\begin{array}{c}0.074^{* *} \\
(0.029)\end{array}$ & $\begin{array}{l}0.049^{*} \\
(0.027)\end{array}$ & & \\
\hline $\begin{array}{l}\text { TB dispensary } \\
\text { Pre-int. } \text { Gini }_{c}\end{array}$ & & & & & & & $\begin{array}{c}0.009 \\
(0.052)\end{array}$ & $\begin{array}{l}-0.038 \\
(0.044)\end{array}$ \\
\hline Avg. TB rate & 1.306 & 1.034 & 1.304 & 1.034 & 1.306 & 1.034 & 1.309 & 1.034 \\
\hline City FE & Yes & Yes & Yes & Yes & Yes & Yes & Yes & Yes \\
\hline Year FE & Yes & Yes & Yes & Yes & Yes & Yes & Yes & Yes \\
\hline Disease FE & No & Yes & No & Yes & No & Yes & No & Yes \\
\hline Disease $\mathrm{FE} \times$ Year FE & No & Yes & No & Yes & No & Yes & No & Yes \\
\hline Disease FE $\times$ City FE & No & Yes & No & Yes & No & Yes & No & Yes \\
\hline City FE × Year FE & No & Yes & No & Yes & No & Yes & No & Yes \\
\hline Time period & 1890-1939 & 1901-1939 & 1890-1939 & 1901-1939 & 1890-1939 & 1901-1939 & 1890-1939 & 1901-1939 \\
\hline Observations & 3,981 & 25,320 & 3,981 & 25,320 & 3,981 & 25,320 & 3,341 & 20,992 \\
\hline R-squared & 0.53 & 0.70 & 0.53 & 0.70 & 0.53 & 0.70 & 0.51 & 0.69 \\
\hline Cities & 87 & 87 & 87 & 87 & 87 & 87 & 68 & 68 \\
\hline
\end{tabular}

Notes: The table reports least squares estimates. In columns (1), (3), (5), and (7) the left-hand-side variable is the TB mortality per 1,000 people, and in columns (2), (4), (6), and (8) the left-hand-side variable is the stacked causes of death from TB, cancer, influenza, pneumonia, accidents and suicides (including homicides from 1931), scarlet fever, diphtheria, and polio per 1,000 people. All regressions include city and year fixed effects, and addtionally columns (2), (4), (6), and (8) include disease, disease-by-year, disease-by-city, and city-by-year fixed effects. TB dispensary ${ }_{c, t}$ is an indicator variable equal to one after the introduction of a TB dispensary multiplied by an indicator equal to one if the disease on the left-hand side is TB. Initial TB rate ${ }_{c}$, Pre-int. pop. density ${ }_{c}$, Pre-int. $\log \left(\right.$ income $_{c}$, and Pre-int. Gini ${ }_{c}$ are the 1890 TB mortality per 1,000 people, the 1890 population per 1890 acreage of the city, the 1904-05 log income per capita, and the 1918-19 income distribution-based Gini coefficient, respectively, all normalized to have a zero mean and a standard deviation of one. For cities included in the data set later than 1890, 1904, and 1918, we use the TB mortality rate, income per taxpayer, and Gini coefficients in the year they are added to the data set. The acreage of the previously German cities is from 1920. In columns (7) and (8) cities that adopted a TB dispensary before 1920 are excluded from the sample. Robust standard errors clustered at the city level are in parentheses.

${ }^{*},{ }^{* *}$ and ${ }^{* * *}$ determine significance levels of 10 percent, 5 percent and 1 percent, respectively. Electronic copy available at: https://ssrn.com/abstract=3036887 
Table 10: Effect of the TB dispensaries using urban/rural-by-month-by-disease data

\begin{tabular}{lc}
\hline \hline Dep. variable: & Disease \\
\cline { 2 - 2 } & $(1)$ \\
\hline TB dispensaries \\
& $-1.774^{* * *}$ \\
& $(0.585)$ \\
\hline Avg. TB deaths & 103.519 \\
\hline Disease FE $\times$ Urban FE $\times$ Month FE & Yes \\
Disease FE $\times$ Urban FE $\times$ Year FE & Yes \\
Disease FE $\times$ Month FE $\times$ Year FE & Yes \\
Urban FE $\times$ Month FE $\times$ Year FE & Yes \\
\hline Time period & $1920 \mathrm{~m} 1-1939 \mathrm{~m} 12$ \\
Observations & 5,280 \\
R-squared & 0.99 \\
\hline
\end{tabular}

Notes: The table reports least squares estimates. In column (1) the left-hand-side variable is the stacked causes of death from TB, measles, scarlet fever, alcohol poisoning, accidents and homicides, suicides, diphtheria, typhoid fever, puerperal fever, pneumonia, and cancer, varying at the monthly level, and by rural and urban areas from January 1920 to December 1939. All regressions include disease, urban, month, year, disease-byurban-by-month, disease-by-urban-by-year, disease-by-month-by-year, and urban-by-month-by-year fixed effects. TB dispensaries s,t $_{u}$ is the stacked number of TB dispensaries in the rural or urban areas at time $t$ multiplied by an indicator equal to one if the disease on the left-hand side is TB. Robust standard errors clustered at the rural/urban by disease level are in parentheses.

${ }^{*},{ }^{* *}$ and ${ }^{* * *}$ determine significance levels of 10 percent, 5 percent and 1 percent, respectively. 
Table 11: Effect of TB dispensaries on patients in sanatoria and TB hospitals

\begin{tabular}{|c|c|c|c|c|}
\hline \multirow[t]{2}{*}{ Dep. variable: } & $\begin{array}{l}\log (\text { patients } \\
\text { in sanatoria) }\end{array}$ & $\begin{array}{l}\log (\text { patients in } \\
\text { sanatoria rate })\end{array}$ & $\begin{array}{c}\log (\text { patients in } \\
\text { TB hospitals })\end{array}$ & $\begin{array}{c}\log (\text { patients in } \\
\text { TB hospitals rate })\end{array}$ \\
\hline & $(1)$ & $(2)$ & $(3)$ & $(4)$ \\
\hline TB dispensary ditt $_{1}$ & $\begin{array}{c}-0.082^{* *} \\
(0.033)\end{array}$ & $\begin{array}{c}-0.099^{* * *} \\
(0.034)\end{array}$ & $\begin{array}{c}0.055 \\
(0.070)\end{array}$ & $\begin{array}{c}0.041 \\
(0.070)\end{array}$ \\
\hline Avg. dep. var. & 5.634 & 0.702 & 4.607 & -0.365 \\
\hline County FE & Yes & Yes & Yes & Yes \\
\hline Year FE & Yes & Yes & Yes & Yes \\
\hline Time period & 1901-1939 & 1901-1939 & 1908-1939 & 1908-1939 \\
\hline Observations & 488 & 488 & 504 & 504 \\
\hline R-squared & 0.886 & 0.838 & 0.806 & 0.657 \\
\hline Counties & 18 & 18 & 17 & 17 \\
\hline
\end{tabular}

Notes: The table reports least squares estimates at the county level. In column (1) the left-hand-side variable is the log of patients in santoria, in column (2) the left-hand-side variable is the log of patients in sanatoria per 1,000 people, in column (3) the left-hand-side variable is the log of patients in TB hospitals, and in column (4) the left-hand-side variable is the log of patients in TB hospitals per 1,000 people. The number of patients in any sanatoria are not available for the year 1928. All regressions include county and year fixed effects. TB dispensary ${ }_{a, t}$ is the number of TB dispensaries in county $a$ at time $t$. Robust standard errors clustered at the county level are in parentheses.

${ }^{*},{ }^{* *}$ and ${ }^{* * *}$ determine significance levels of 10 percent, 5 percent and 1 percent, respectively. 
Table 12: Costs and benefits of TB dispensaries

\begin{tabular}{lccc}
\hline \hline & Point estimate & $95 \%$ CI low & $95 \%$ CI high \\
\hline Deaths averted from 1908 to 1939 & 7,199 & 6,123 & 8,275 \\
Life-years saved from 1908 to 1939 & 284,403 & 241,891 & 326,915 \\
Cost of TB dispensaries from 1908 to 1939 & $19,210,792$ & & \\
Cost per averted death & 2,668 & 3,137 & 2,321 \\
Cost per life-year saved & 68 & 79 & 59 \\
Benefits of TB dispensaries from 1908 to 1939 & $1,984,327,876$ & $1,687,715,547$ & $2,280,940,205$ \\
Social rate of return & $103: 1$ & $88: 1$ & $119: 1$ \\
\hline
\end{tabular}

Notes: The table reports costs and benefits of the TB dispensaries. Based on the baseline difference-in-differences estimate in Table 3, column (1) the number of deaths averted by TB dispensaries commissioned between 1908 and 1939 is predicted. The life years saved are calculated by weighting the age distribution of TB mortality from 1921 to 1925 by the age-specific life expectancy of Denmark in each year from 1908 to 1939. The total costs of the TB dispensaries from 1908 to 1939 are estimated by the yearly per capita cost in 2015 US dollars implied by the financial accounts of nine TB dispensaries. The cost per averted death and the cost per life year saved are estimated by dividing the deaths averted and the life years saved by the total costs, respectively. The benefits of the TB dispensaries are calculated by multiplying the 1908 value of a life year (VOLY) by the number of deaths averted by the TB dispensaries. The 1908 VOLY is derived from the CES function $V O L Y_{1908}=V O L Y_{2015}\left(Y_{1908} / Y_{2007}\right)^{\epsilon}$, where $\epsilon$ is the income elasticity assumed to be 1.7 , and $Y_{t}$ is the income per capita peaking in 2007 before the Great Recession. The 2015 VOLY is assumed to be 190,000 US dollars. The social rate of return is calculated by dividing the total benefits with the tocal costs. 
Table 13: Productivity effects of TB dispensaries

\begin{tabular}{lcc}
\hline \hline Dep. variable: & \multicolumn{2}{c}{$\log ($ income pr. taxpayer $)$} \\
\cline { 2 - 3 } & $(1)$ & $(2)$ \\
\hline TB dispensary & \\
& $0.028^{*}$ & \\
TB dispensary & & \\
& $(0.016)$ & \\
& & $0.004^{* * *}$ \\
City FE & & $(0.002)$ \\
Year FE & Yes & Yes \\
\hline Time period & Yes & Yes \\
Observations & $1904-1939$ & $1904-1939$ \\
R-squared & 2,940 & 2,940 \\
Cities & 0.84 & 0.84 \\
\hline
\end{tabular}

Notes: The table reports least squares estimates. In columns (1) and (2) the left-hand-side variable is the log income per taxpayer. All regressions include city and year fixed effects. TB dispensary ${ }_{c, t}$ is an indicator variable equal to one after the introduction of a TB dispensary, $j_{c}$ is the year of the introduction of a dispensary, and $t$ is the year. Robust standard errors clustered at the city level are in parentheses.

${ }^{*},{ }^{* *}$ and ${ }^{* * *}$ determine significance levels of 10 percent, 5 percent and 1 percent, respectively. 


\title{
Online Appendix:
}

\section{Preventing the White Death: Tuberculosis Dispensaries}

\author{
Casper Worm Hansen Peter Sandholt Jensen* Peter Egedesø Madsen
}

September 2017

*Contact: Casper Worm Hansen, University of Copenhagen, casper.worm.hansen@econ.ku.dk, Peter Sandholt Jensen, University of Southern Denmark, psj@sam.sdu.dk, Peter Egedes $\varnothing$ Madsen, University of Southern Denmark, pe@sam.sdu.dk 


\section{Mortality by cause and age in Danish cities}

This section outlines the aggregate city development of various cause-specific mortality rates (from 1901 to 1950), as well as aggregate TB incidence (from 1920 to 1950) and the age distribution of TB mortality in 1921-1925.

Figure A1: Pulmonary TB mortality in the Danish cities, 1901-1950

Figure A2: Pneumonia mortality in the Danish cities, 1901-1950

Figure A3: Scarlet fever mortality in the Danish cities, 1901-1950

Figure A4: Cancer mortality in the Danish cities, 1901-1950

Figure A5: Accident and suicide mortality in the Danish cities, 1901-1950

Figure A6: Polio mortality in the Danish cities, 1901-1950

Figure A7: Influenza mortality in the Danish cities, 1901-1950

Figure A8: Diphtheria mortality in the Danish cities, 1901-1950

Figure A9: Mortality in the Danish cities, 1890-1950

Figure A10: Child mortality in the Danish cities, 1901-1950

Figure A11: Live births in the Danish cities, 1901-1950

Figure A12: Pulmonary TB morbidity and mortality in Denmark, 1921-1949

Figure A13: Pulmonary TB mortality rates by gender in Copenhagen and the other Danish cities

Figure A14: Age distribution of TB mortality, 1921-25

Figure A15: Monthly TB mortality in urban and rural areas in the 1920's and 1930's 


\section{Theoretical framework}

This section provides supporting information to the theoretical framework (i.e., the modified SIR model) discussed in the paper. A summary of the model's equations (as outlined in the paper) is given here:

$$
\begin{gathered}
S_{t+1}=S_{t}+B_{t}-I_{t}^{N e w}-D_{t}^{\text {other }}+R_{t}, S_{0} \text { given } \\
B_{t}=b S_{t}, \\
D_{t}^{\text {other }}=\lambda^{D} S_{t}, \\
I_{t}^{N e w}=\lambda_{t}^{I} S_{t}, \\
\lambda_{t}^{I}=\frac{\beta}{y_{t}^{\gamma}}\left(\frac{I_{t}}{S_{t}}\right)^{\alpha}, \\
(\sigma-\theta)(1-\delta) \\
y_{t+1}\left(1+g_{y}\right) y_{t}, y_{0} \text { given, } \\
R_{t}=\lambda_{t}^{R} I_{t}, \\
\lambda_{t}^{R}=\bar{\lambda}^{R}-\frac{\Psi}{y_{t}}, \\
I_{t+1}=I_{t}+I_{t}^{N e w}-D_{t}^{T B}-R_{t}, I_{0} \text { given, } \\
D_{t}^{T B}=\lambda^{T B} I_{t} .
\end{gathered}
$$

Our next step is to solve the model. First, using these equations yields the following system of three first-order difference equations, which fully characterize how the populations and income evolve over time:

$$
\begin{aligned}
S_{t+1} & =\left(1+b-\lambda^{D}\right) S_{t}-\frac{\beta}{y_{t}^{\gamma}}\left(\frac{I_{t}}{S_{t}}\right)^{\alpha} S_{t}+\left(\bar{\lambda}_{t}^{R}-\frac{\Psi}{y_{t}}\right) I_{t}, S_{0} \text { given, } \\
I_{t+1} & =\left(1-\lambda^{T B}-\left(\bar{\lambda}^{R}-\frac{\Psi}{y_{t}}\right)\right) I_{t}+\frac{\beta}{y_{t}^{\gamma}}\left(\frac{I_{t}}{S_{t}}\right)^{\alpha} S_{t}, I_{0} \text { given, } \\
y_{t+1} & =\left(1+g_{y}\right) y_{t}, y_{0} \text { given. }
\end{aligned}
$$


Next, assume that $g_{y}=0, y_{t+1}=y_{t}=y$, and rewrite the system of the two remaining first order equations in terms of $s_{t} \equiv I_{t} / S_{t}$. We start by the difference equation for $I_{t}$ :

$$
\begin{aligned}
I_{t+1} & =\left(1-\lambda^{T B}-\left(\bar{\lambda}^{R}-\frac{\Psi}{y}\right)\right) I_{t}+\frac{\beta}{y^{\gamma}}\left(\frac{I_{t}}{S_{t}}\right)^{\alpha} S_{t} \Leftrightarrow \\
G_{t}^{I} & \equiv \frac{I_{t+1}}{I_{t}}=\left(1-\lambda^{T B}-\left(\bar{\lambda}^{R}-\frac{\Psi}{y}\right)\right)+\frac{\beta}{y^{\gamma}}\left(\frac{S_{t}}{I_{t}}\right)^{1-\alpha} \Leftrightarrow \\
G_{t}^{I} & =1-\lambda^{T B}-\bar{\lambda}^{R}+\frac{\Psi}{y}+\frac{\beta}{y^{\gamma}} s_{t}^{\alpha-1} .
\end{aligned}
$$

And now the difference equation for $S_{t}$ :

$$
\begin{aligned}
S_{t+1} & =\left(1+b-\lambda^{D}\right) S_{t}-\frac{\beta}{y^{\gamma}}\left(\frac{I_{t}}{S_{t}}\right)^{\alpha} S_{t}+\left(\bar{\lambda}_{t}^{R}-\frac{\Psi}{y}\right) I_{t} \Leftrightarrow \\
G_{t}^{S} & \equiv \frac{S_{t+1}}{S_{t}}=\left(1+b-\lambda^{D}-\frac{\beta}{y^{\gamma}}\left(\frac{I_{t}}{S_{t}}\right)^{\alpha}+\left(\bar{\lambda}_{t}^{R}-\frac{\Psi}{y}\right) \frac{I_{t}}{S_{t}}\right) \Leftrightarrow \\
G_{t}^{S} & =1+b-\lambda^{D}-\frac{\beta}{y^{\gamma}} s_{t}^{\alpha}+\left(\bar{\lambda}_{t}^{R}-\frac{\Psi}{y}\right) s_{t} .
\end{aligned}
$$

Equations (2) and (3) can now be depicted in a $G, s$-diagram. If these two curves intersect, $S$ and $I$ are growing at the same rate and $s$ becomes constant. Next, equation (2) shows that if $\alpha<1$ i) $G_{t}^{I}$ is falling in $s$; ii) $G_{t}^{I} \rightarrow \infty$ when $s \rightarrow 0$; and iii) $G_{t}^{I} \rightarrow 1-\lambda^{T B}-\bar{\lambda}^{R}$ as $s \rightarrow \infty$. From equation (3), we see that i) $G_{t}^{S}$ is decreasing in $s$ if $s<\tilde{s}$, where:

$$
\tilde{s} \equiv\left(\frac{\alpha \beta}{y^{\gamma}\left(\bar{\lambda}_{t}^{R}-\frac{\Psi}{y}\right)}\right)^{\frac{1}{1-\alpha}}
$$

and increasing when $s>\tilde{s}$; ii) $G_{t}^{S}(0)=1+b-\lambda^{D}$; and iii) $G_{t}^{I} \rightarrow \infty$ when $s \rightarrow \infty$. Now, assuming that $1-\lambda^{T B}-\bar{\lambda}^{R}+\frac{\Psi}{y}>1+b-\lambda^{D}-\frac{\beta}{y^{\gamma}} \tilde{s}^{\alpha}+\left(\bar{\lambda}_{t}^{R}-\frac{\Psi}{y}\right) \tilde{s}$, we know that (3) for sure intersects (2) from below, and we are guaranteed one global stable steady state as, in this case, if $s^{\prime}>s^{*}$ then $G_{t}^{S}>G_{t}^{I}$ and $S$ is growing more than $I_{t}$, implying that $s_{t} \equiv S_{t} / I_{t}$ falls until $s^{\prime}=s^{*}$. Likewise, if $s^{\prime}<s^{*}$ then $G_{t}^{S}<G_{t}^{I}$ and $S$ is growing less than $I_{t}$, implying that $s_{t} \equiv S_{t} / I_{t}$ increases until $s=s^{*}$. In other words, if $s^{*}>\tilde{s}, s^{*}$ is a stable steady state. Figure A16 depicts (2) and (3) under the above-mentioned parameter restrictions, and it shows that $s^{*}$ is in fact globally stable. 


\section{Figure A16: $G, s$-diagram}

From the position of the two curves (2 and 3 ) in Figure A16, we see how $s^{*}$ changes when

various parameters of the model are changed: $\frac{d s^{*}}{d b}>0, \frac{d s^{*}}{d \lambda^{D}}<0, \frac{d s^{*}}{d \lambda^{T B}}<0, \frac{d s^{*}}{d \lambda^{R}}<0, \frac{d s^{*}}{d y}<0$, $\frac{d s^{*}}{d \theta}<0$, and $\frac{d s^{*}}{d \delta}<0$. We note that although the TB incidence rate decreases when $\lambda^{T B}$ increases (i.e., $d s^{*} / d \lambda^{T B}<0$ ), the TB mortality rate would still increase. So if one finds empirically that the incidence rate and mortality rates go in opposite directions, this could be evidence of that $\lambda^{T B}$ has changed.

For our simulation exercise, reported in Figure 4 of the paper, we use the following set of parameter values:

$$
\begin{aligned}
S_{0} & =14,000 ; I_{0}=293.95 ; b=0.0281 ; \lambda^{D}=0.013661 ; \lambda^{T B}=0.08 \\
\alpha & =\gamma=0.5 ; y_{0}=1 ; g_{y}=0.015 ; \Psi=0.1 ; \sigma=0.05 ; \theta_{1}=0 ; \delta=0
\end{aligned}
$$

The values of $S_{0}, I_{0}, b, \lambda^{D}$ are set so as to roughly match first moments in our data in 1908 in terms of population size, TB death rate, crude birth rate, and crude death rate. Using Danish TB mortality data for men above the age of 20 at the turn of the 20th century, Heiberg (1902) finds that about 13 percent of all TB deaths occur within the first year, and $\lambda^{T B}$ is accordingly set below this threshold value at 8 percent. The values $\alpha$ and $\gamma$ are set so as to get at baseline path for the TB mortality rate which resembles the observed one. We set $y_{0}=1$ and $\Psi=0.1$ so that we get an annual recovery rate in the interval $[0.4 ; 0.5]$. We could alternatively have set $y_{0}$ to match income per capita from our tax-records data and adjusted $\Psi$ accordingly to get the same interval of recovery rates; of course, this yields the same findings. The average annual growth rate in (tax) income per capita is in the neighborhood of 1.5 percent per year, so $g_{y}=0.015$. Finally, in the baseline, we set $\sigma=0.05, \theta_{1}=0$, and $\delta=0$, such that the baseline infectivity rate of TB is 5 percent. These parameter settings constitute our baseline path for the TB mortality rate. When simulating the introduction of the dispensaries, $\theta_{1}$ is increased from zero to $\theta_{2}=0.01$, which lowers the initial infectivity rate to 4 percent. We choose this magnitude in order to mimic the magnitude of the decline in TB mortality as we observe it in the data; however, we note that it still remains interesting to study the shape of the time path due to this parameter change and to see if it looks at all as the empirical counterpart. 


\section{Data and balancing}

Table A1: Data explanations

Table A2: Number of TB dispensaries and TB mortality over time

Table A3: Sample splits and balance tests

Figure A17: Balancing figure

\section{Additional event-study figures}

Figure A18-A24: Additional event-studies

\section{Additional robustness tests}

Table A4: Effect of TB dispensaries on the TB death ratio and TB in level

Table A5: Event studies of the impact of a TB dispensary

Table A6: Effect of TB dispensaries using city-by-year-by-disease data with alternative disease panels

Table A7: Effect of TB dispensaries; alternative treatment measures

Table A8: Effect of TB dispensaries excluding large cities and early adopters

Table A9: Effect of TB dispensaries controlling for early BCG trials, etc.

Table A10: Effect of TB dispensaries controlling for lagged population, initial population, weighting by city sizes, and linear trend

Table A11: Effect of TB dispensaries controlling for commissioning of waterworks

Table A12: Effect of main and branch TB dispensaries

Table A13: Effect of TB dispensaries in a balanced and extended panel 
Figure A1: Pulmonary TB mortality in the Danish cities, 1901-1950

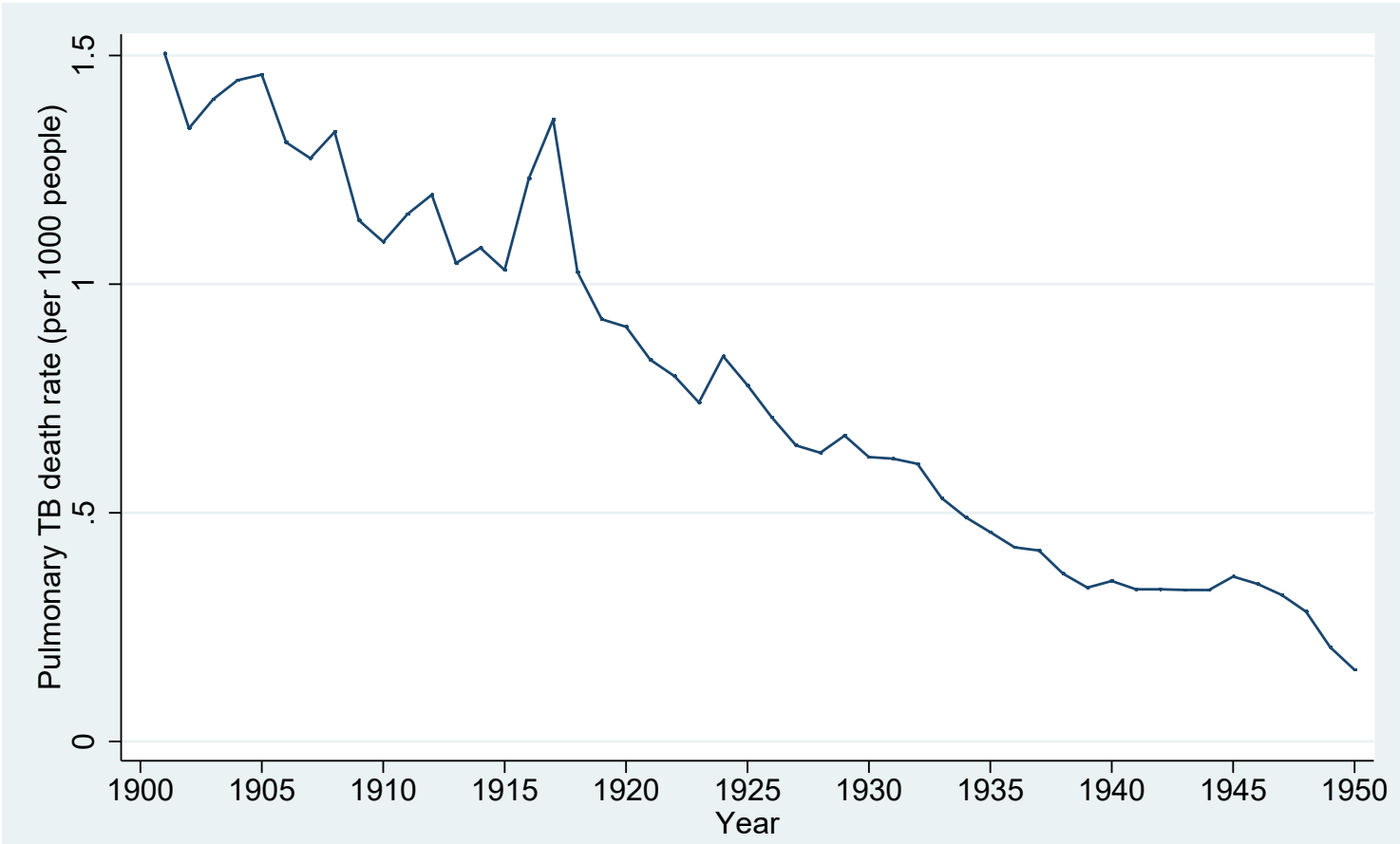

Notes: The graph shows the national development of pulmonary TB mortality in the Danish cities per 1,000 people. Source: The Cause of Death Statistics (1901-1950) and the authors' own calculations. 
Figure A2: Pneumonia mortality in the Danish cities, 1901-1950

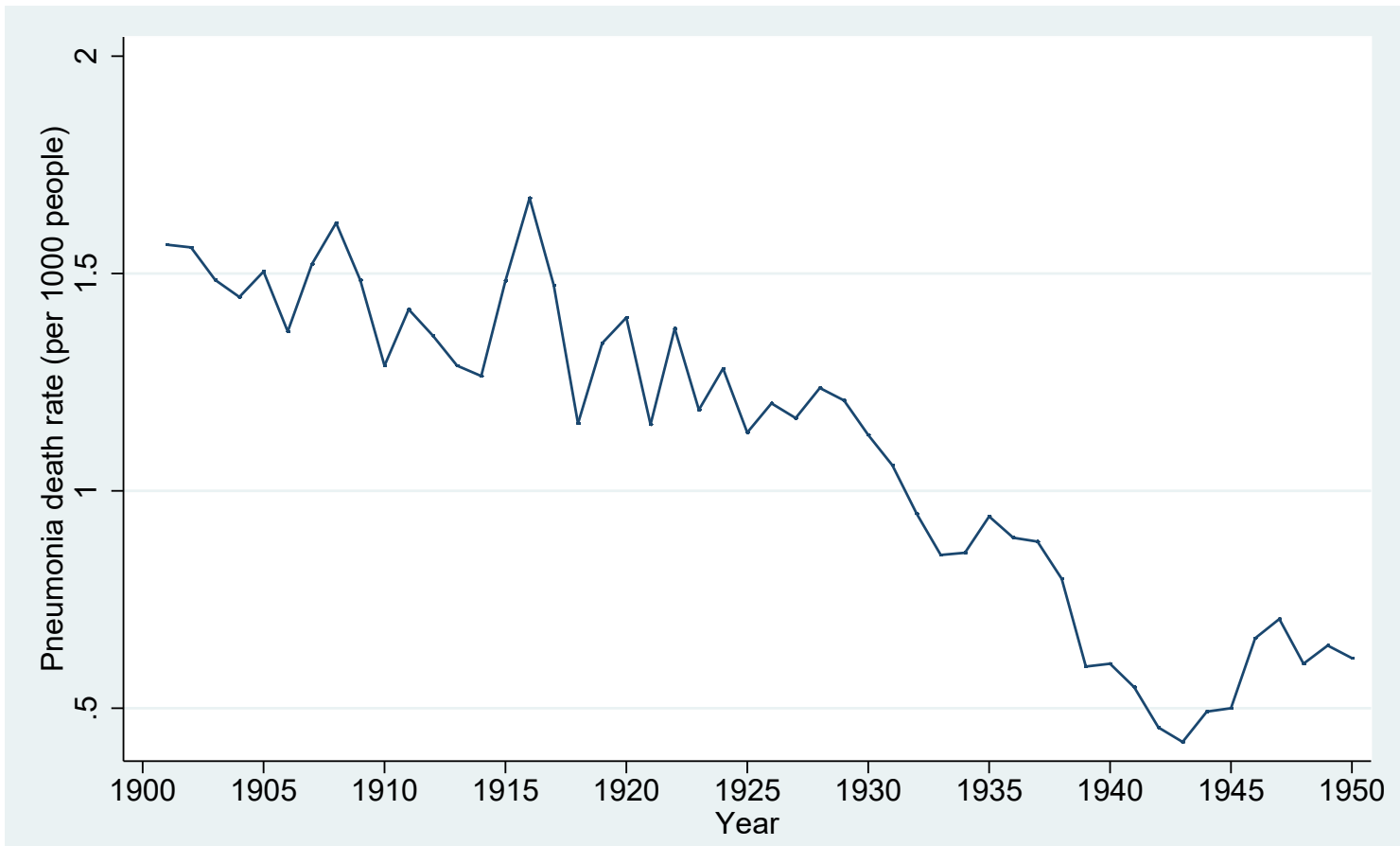

Notes: The graph shows the national development of pneumonia mortality in the Danish cities per 1,000 people. Source: The Cause of Death Statistics (1901-1950) and the authors' own calculations. 
Figure A3: Scarlet fever mortality in the Danish cities, 1901-1950

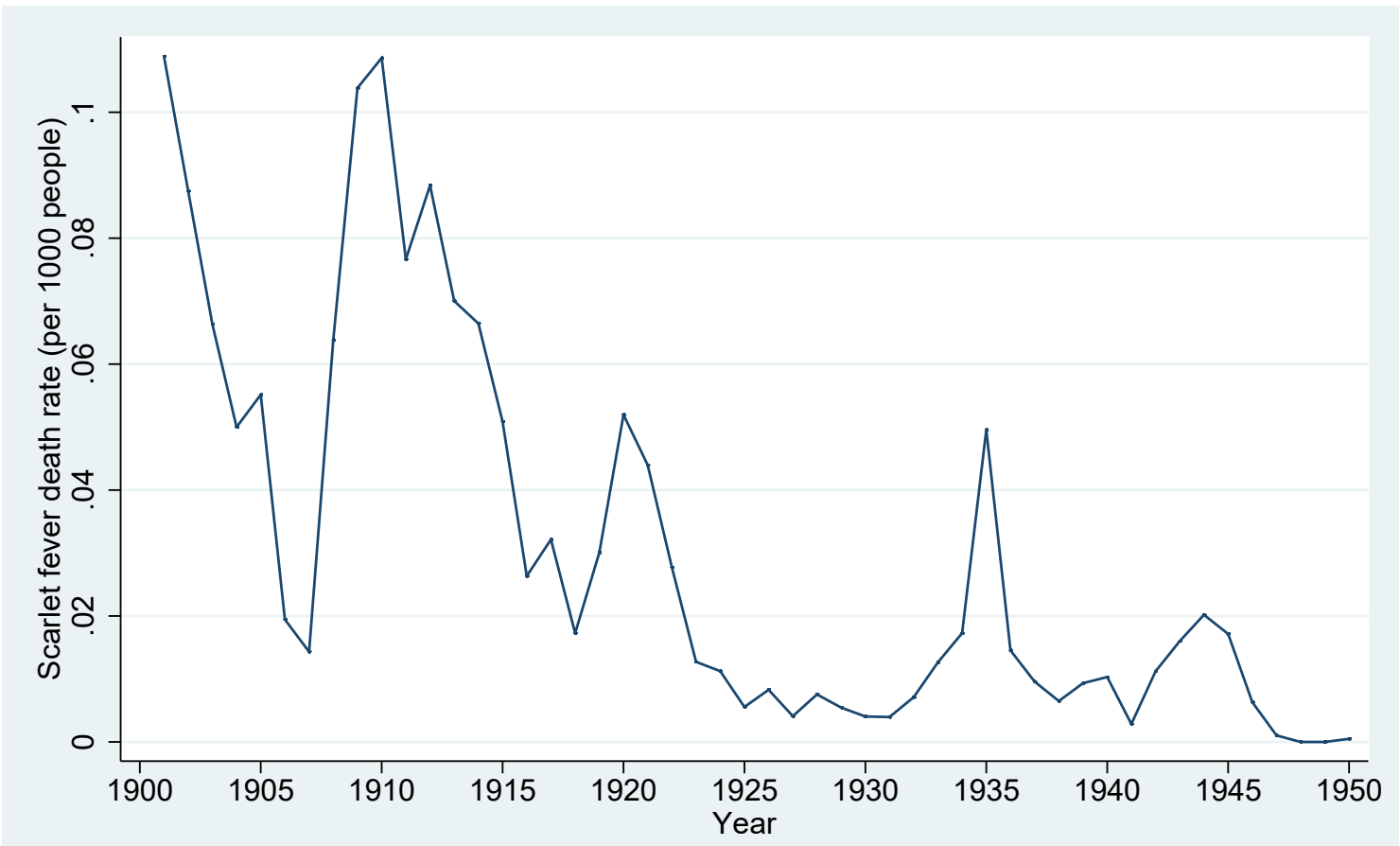

Notes: The graph shows the national development of scarlet fever mortality in the Danish cities per 1,000 people. Source: The Cause of Death Statistics (1901-1950) and the authors' own calculations. 
Figure A4: Cancer mortality in the Danish cities, 1901-1950

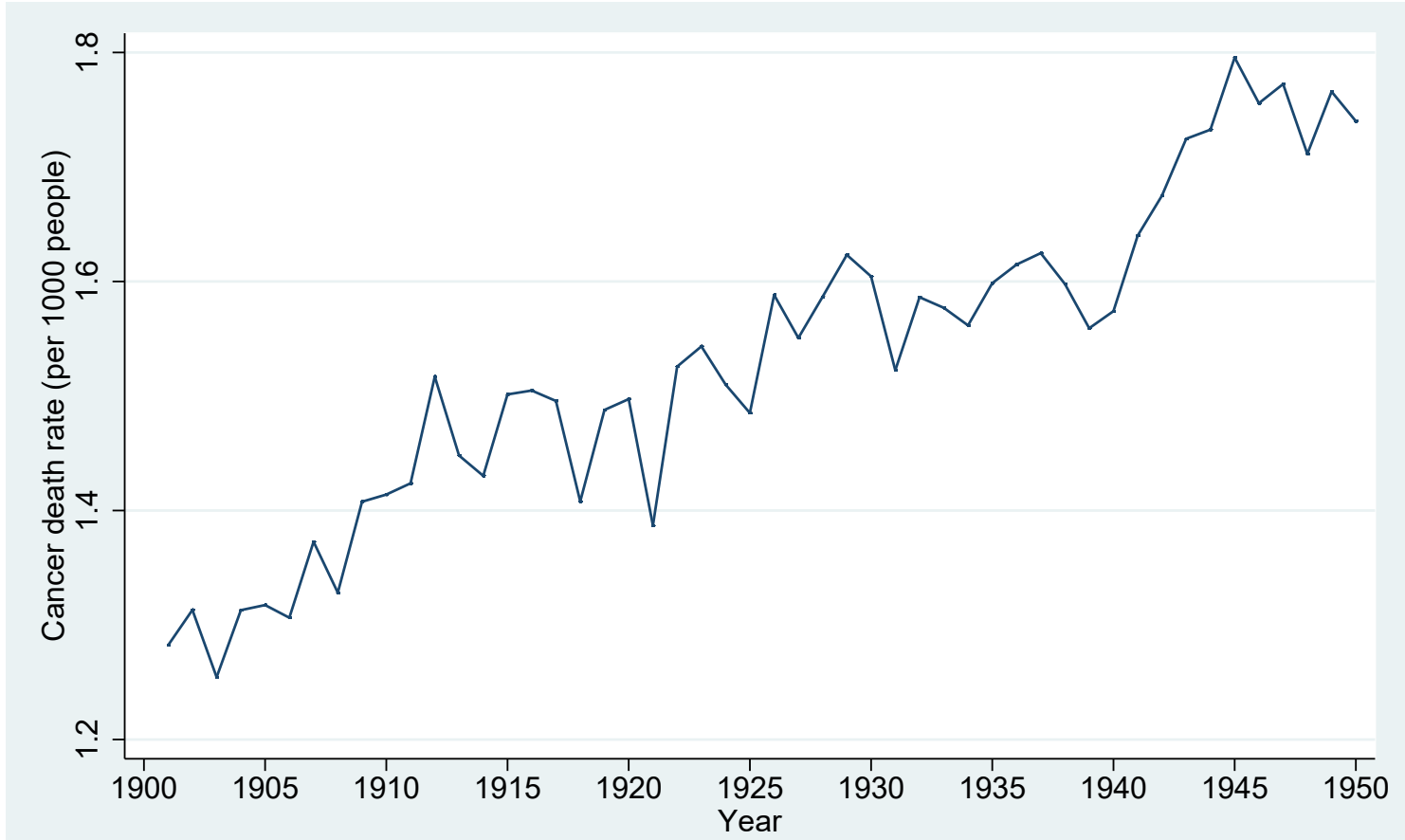

Notes: The graph shows the national development of cancer mortality in the Danish cities per 1,000 people. Source: The Cause of Death Statistics (1901-1950) and the authors' own calculations. 
Figure A5: Accident and suicide mortality in the Danish cities, 1901-1950

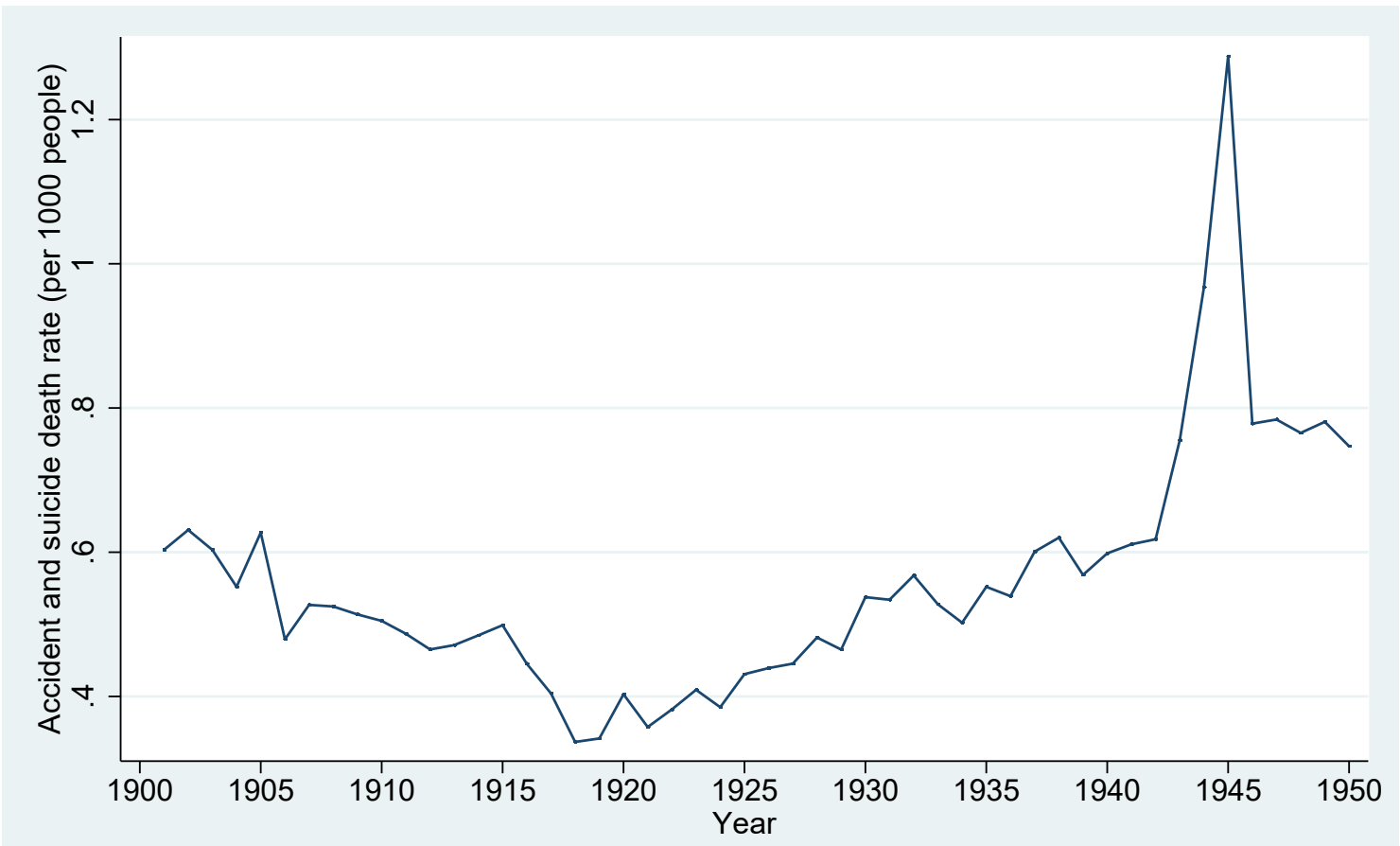

Notes: The graph shows the national development of deaths from accidents and suicides in the Danish cities per 1,000 people. From 1931 the data also include the number of deaths from homicides. Source: The Cause of Death Statistics (1901-1950) and the authors' own calculations. 
Figure A6: Polio mortality in the Danish cities, 1901-1950

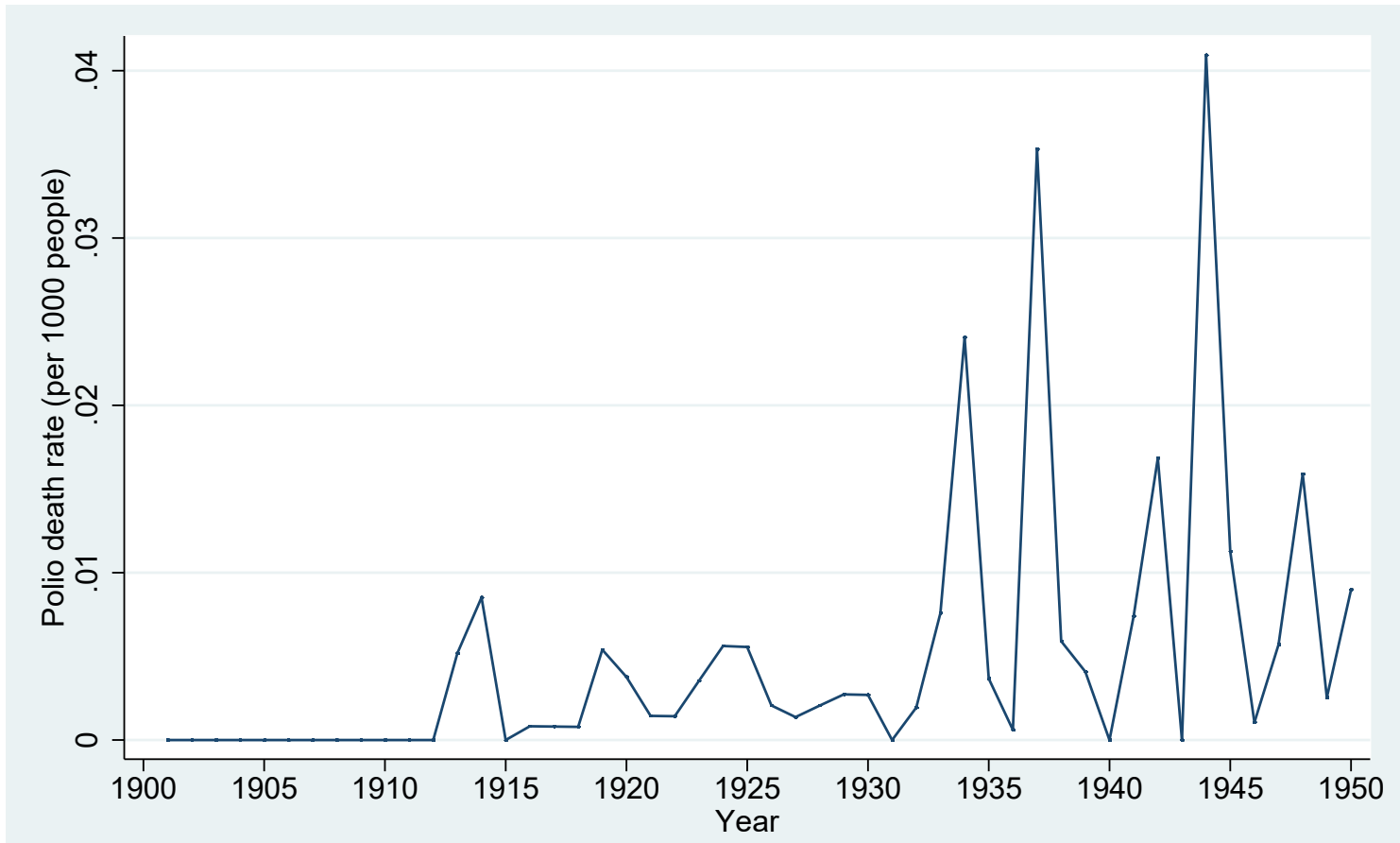

Notes: The graph shows the national development of polio mortality in the Danish cities per 1,000 people. Source: The Cause of Death Statistics (1901-1950) and the authors' own calculations. 
Figure A7: Influenza mortality in the Danish cities, 1901-1950

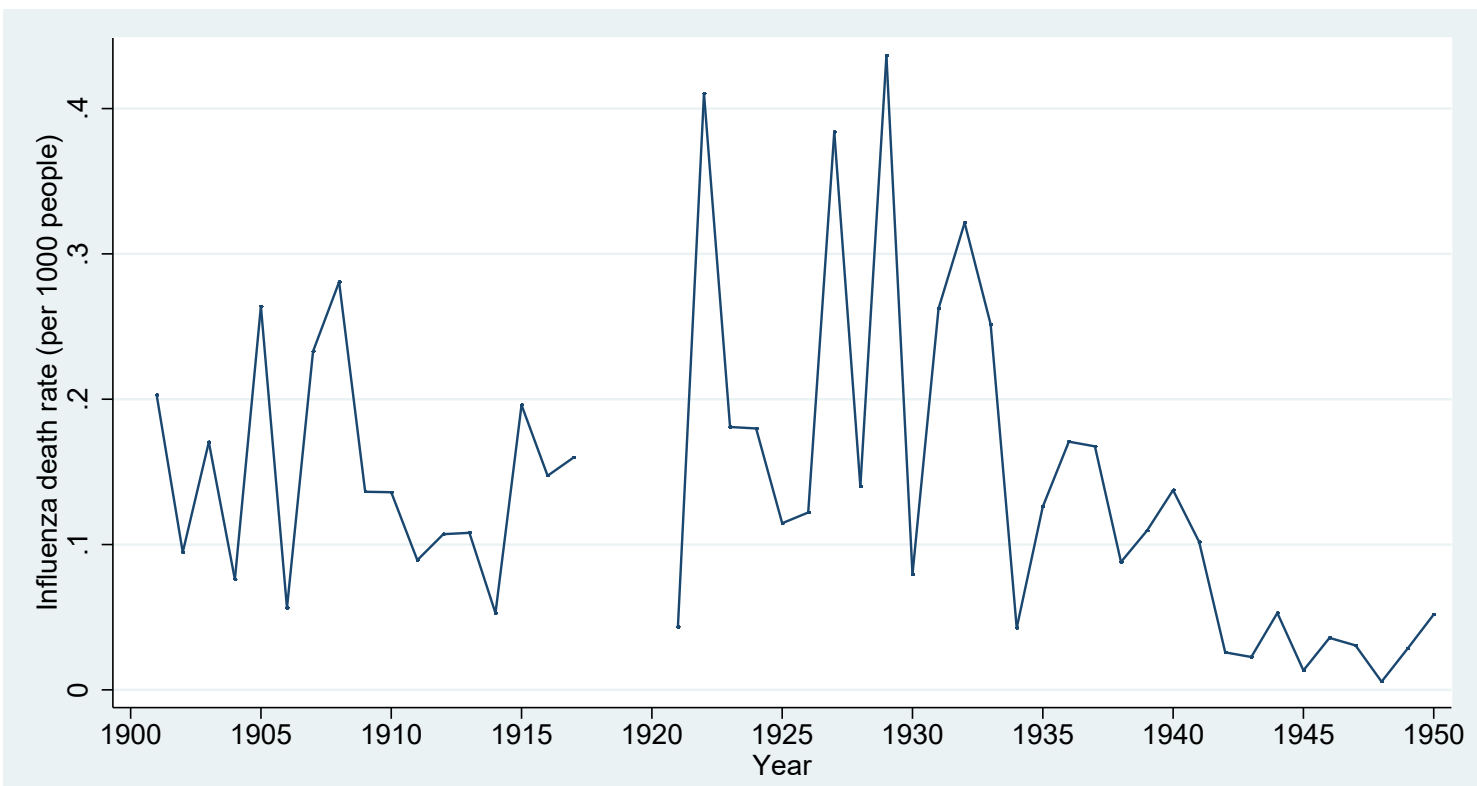

Notes: The graph shows the national development of influenza mortality in the Danish cities per 1,000 people. The years 1918, 1919, and 1920 are not shown on the graph because of high values due to the Spanish flu. The influenza death rates were in the years 1918 to 1920, 3.27, 1.37, and 1.31 respectively. Source: The Cause of Death Statistics (1901-1950) and the authors' own calculations. 
Figure A8: Diphtheria mortality in the Danish cities, 1901-1950

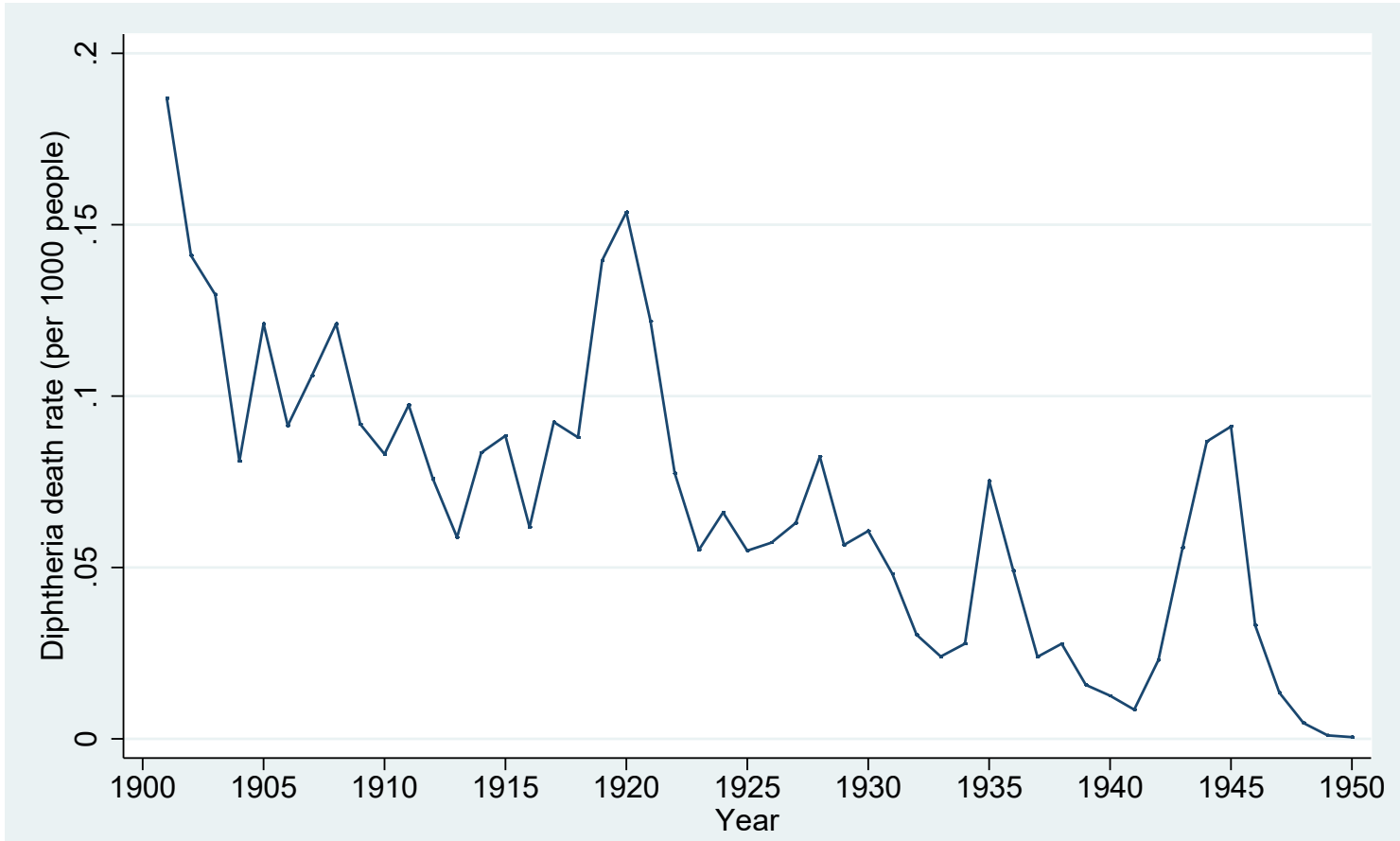

Notes: The graph shows the national development of diphtheria mortality in the Danish cities per 1,000 people. Source: The Cause of Death Statistics (1901-1950) and the authors' own calculations. 
Figure A9: Mortality in the Danish cities, 1890-1950

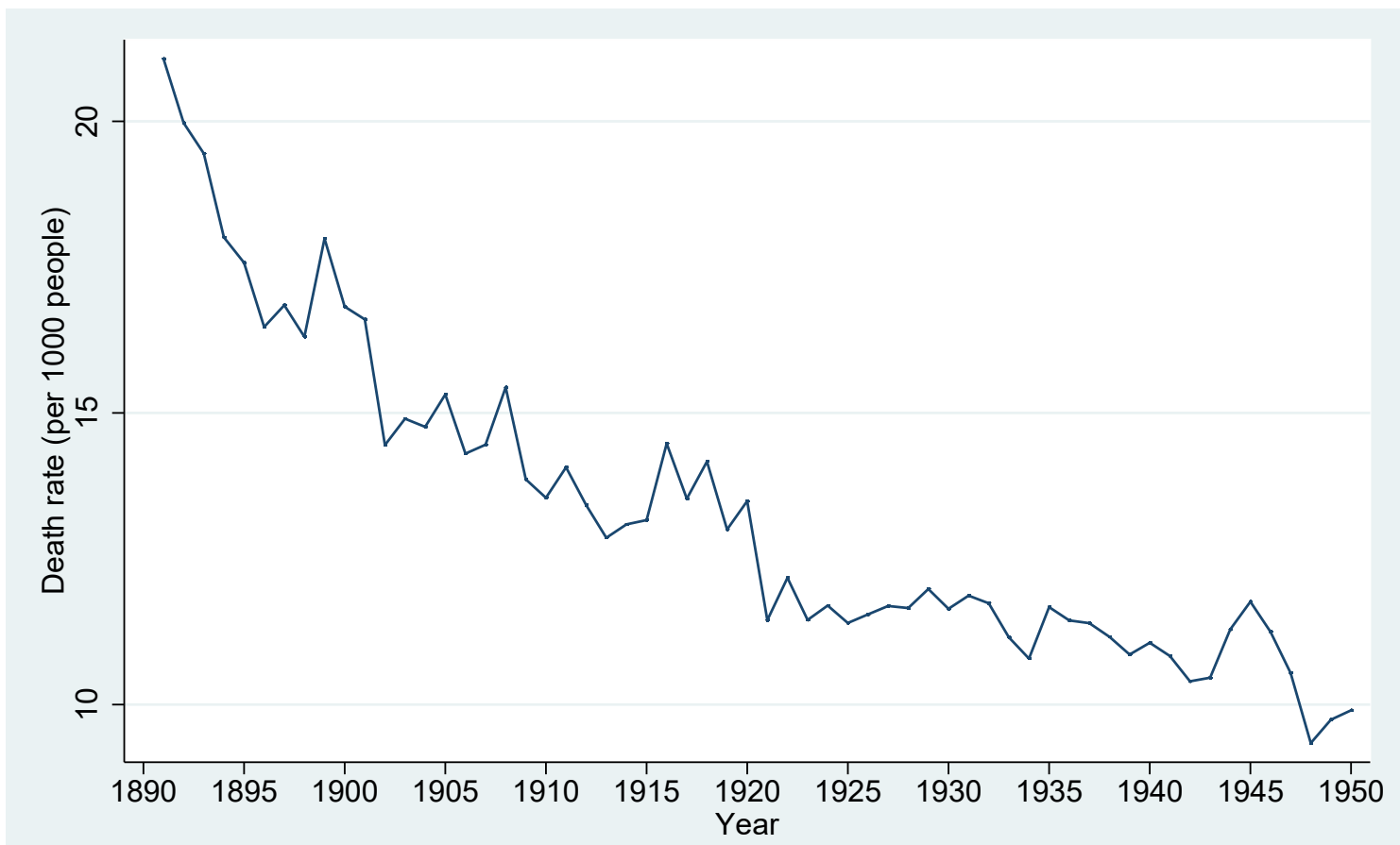

Notes: The graph shows the national development of mortality excluding stillborns in the Danish cities per 1,000 people. Source: The Cause of Death Statistics (1890-1950) and the authors' own calculations. 
Figure A10: Child mortality in the Danish cities, 1901-1950

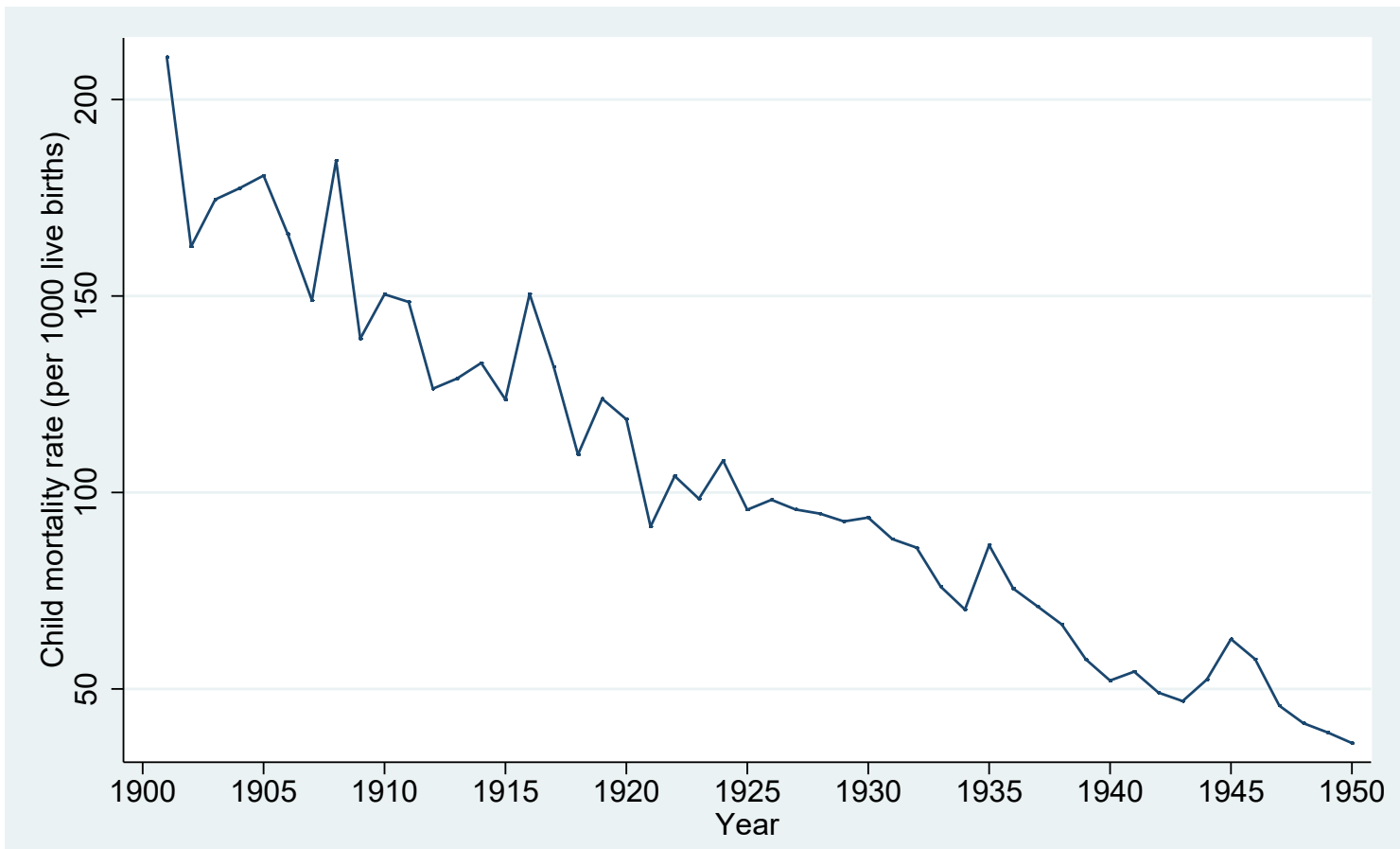

Notes: The graph shows the national development of mortality among 0-5-year-olds in the Danish cities per 1,000 live births from 1901 to 1936 and from 1937 to 1950 the national development of mortality among 0-4-year-olds in the Danish cities per 1,000 live births. Source: The Cause of Death Statistics (1901-1950) and the authors' own calculations. 
Figure A11: Live births in the Danish cities, 1901-1950

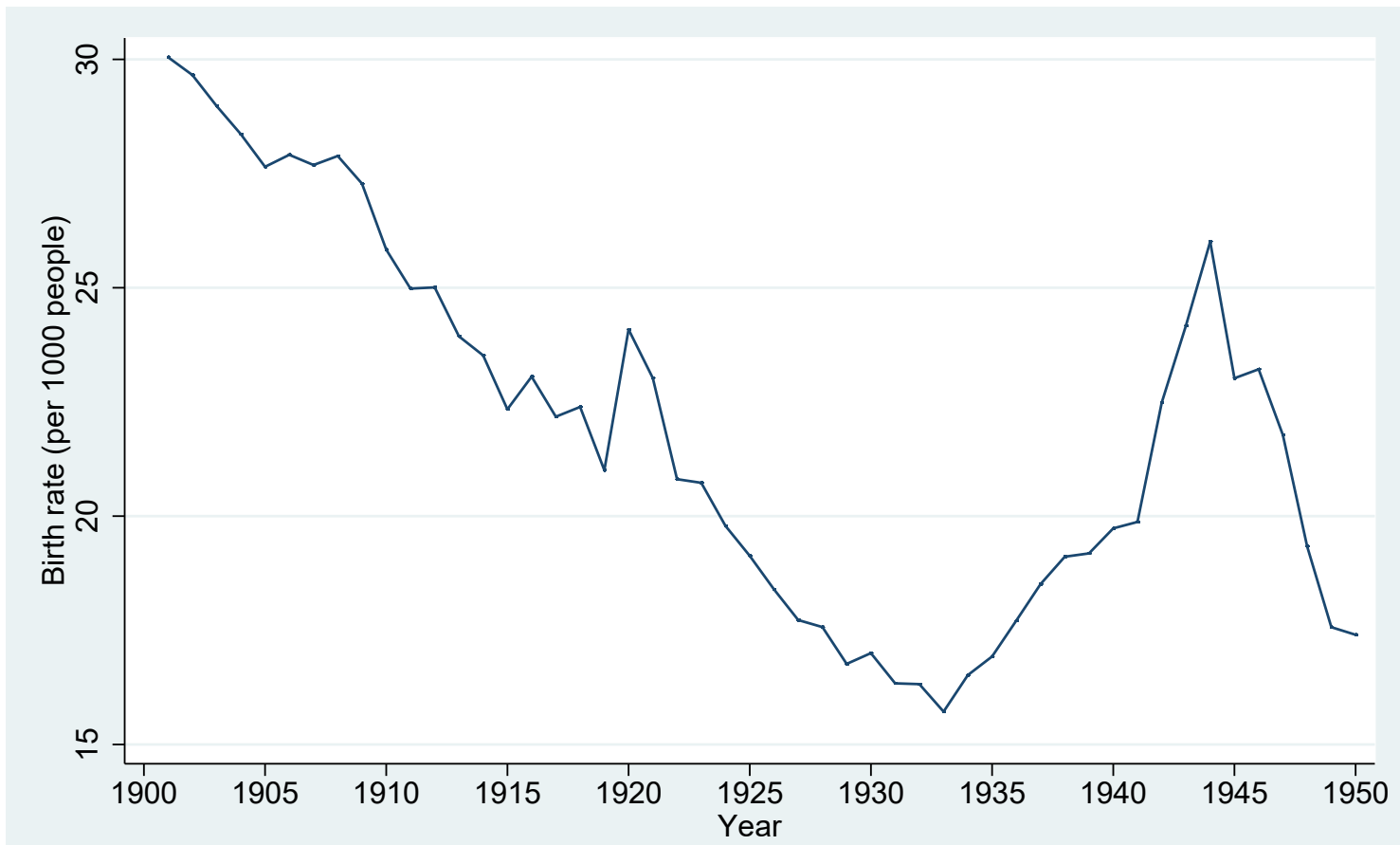

Notes: The graph shows the national development of the birth rate in the Danish cities per 1,000 people. Source: The Cause of Death Statistics (1901-1950) and the authors' own calculations.

Figure A12: Pulmonary TB morbidity and mortality in Denmark, 1921-1949

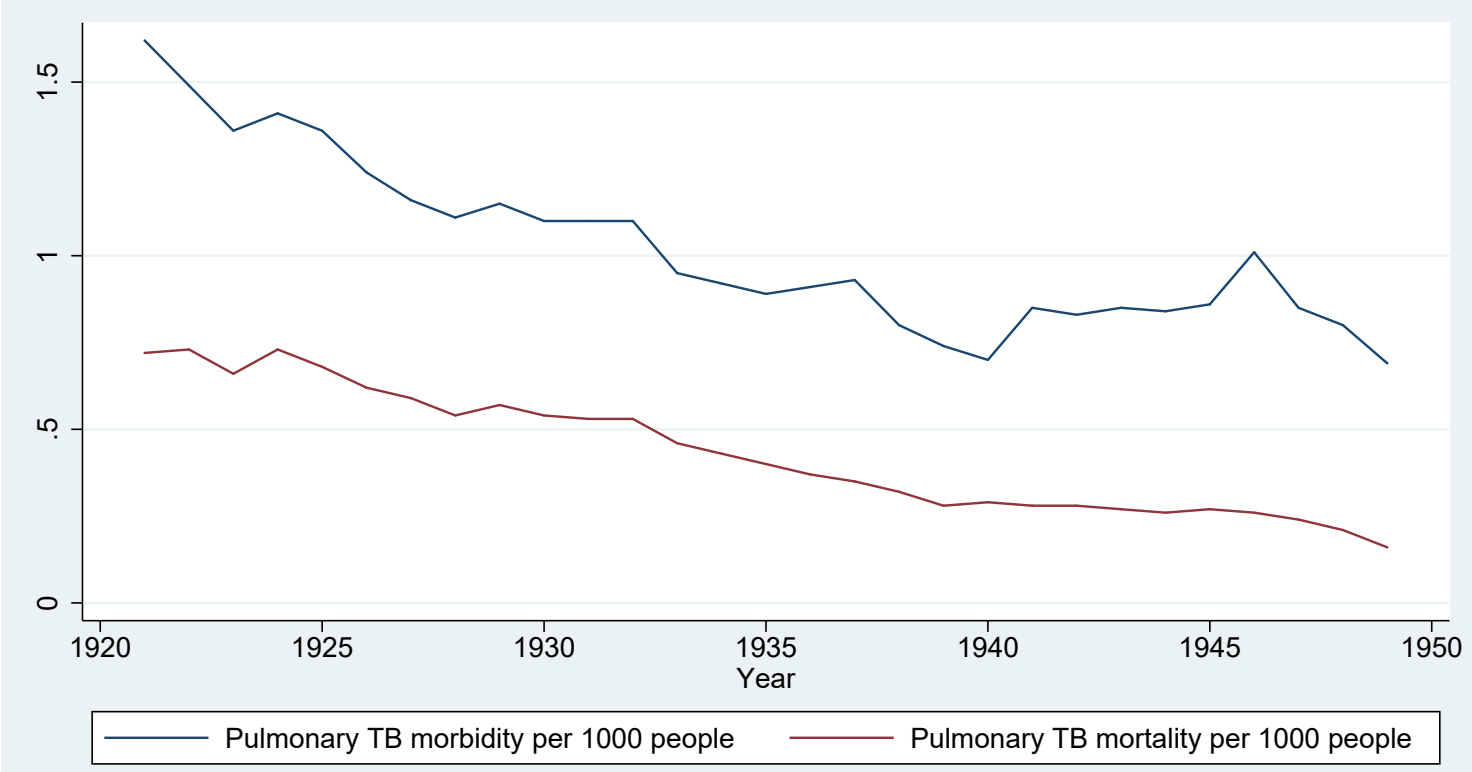

Notes: The graph shows the national development of pulmonary TB morbidity as well as mortality in Denmark per 1,000 people. Source: Schelde Møller (1950, p.145). 
Figure A13: Pulmonary TB mortality rates by gender in Copenhagen and the other Danish cities

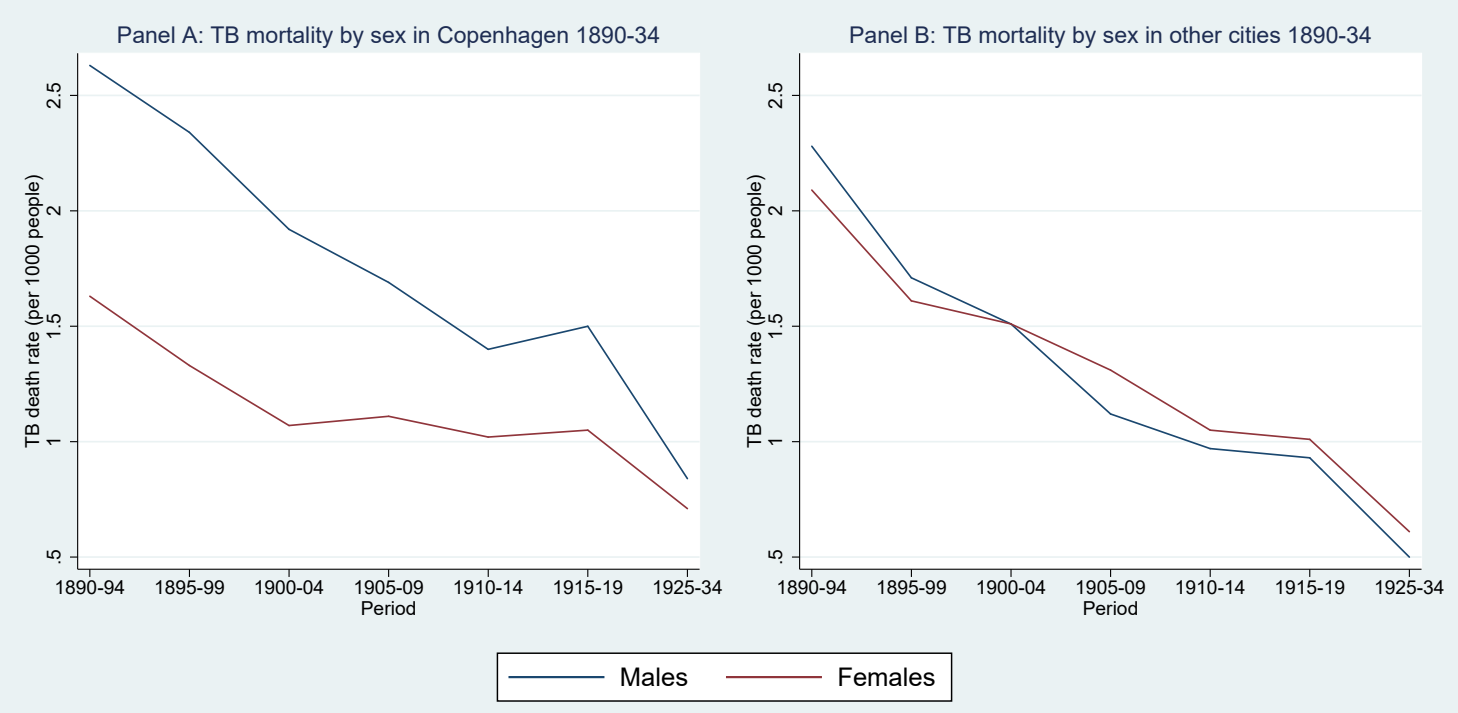

Notes: Panel A depicts pulmonary TB mortality rates by sex in Copenhagen from 1890 to 1934, Panel B depicts pulmonary TB mortality rates by sex in the other Danish cities from 1890 to 1934. Source: Lindhardt (1939) and the authors' own calculations.

Figure A14: Age distribution of TB mortality, 1921-25

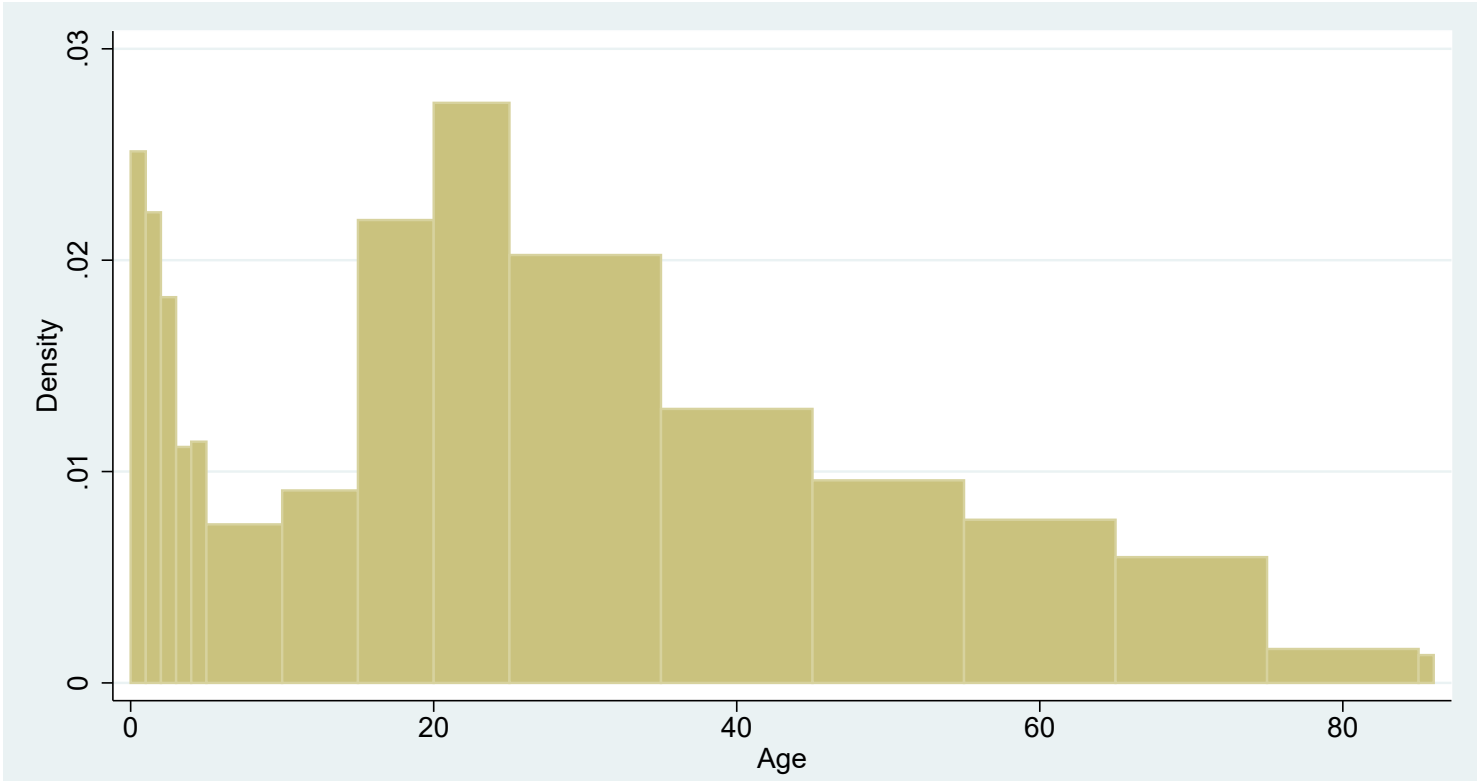

Notes: The graph shows the average age distribution of TB mortality for the five-year period 1921-25. Source: The Cause of Death Statistics (1921-1925) and the authors' own calculations. 
Figure A15: Monthly TB mortality in urban and rural areas in the 1920s and 1930s
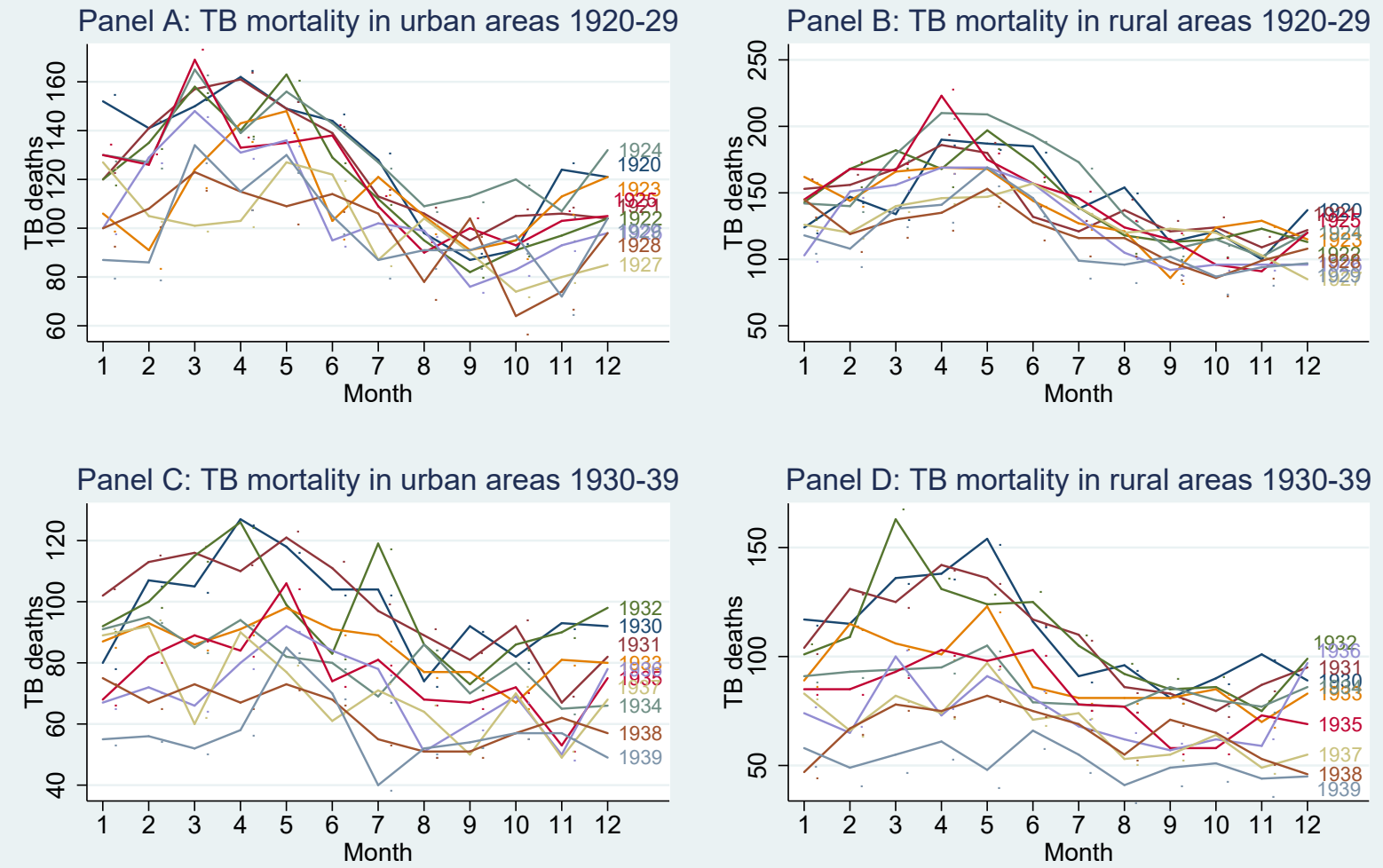

Notes: Panel A depicts the monthly TB mortality in the urban areas in the 1920s, Panel B depicts the monthly TB mortality in the rural areas in the 1920s, Panel C depicts the monthly TB mortality in the urban areas in the 1930s, Panel D depicts the monthly TB mortality in the rural areas in the 1930s. Source: The Cause of Death Statistics (1920-1939). 
Figure A16: $G, s$-diagram

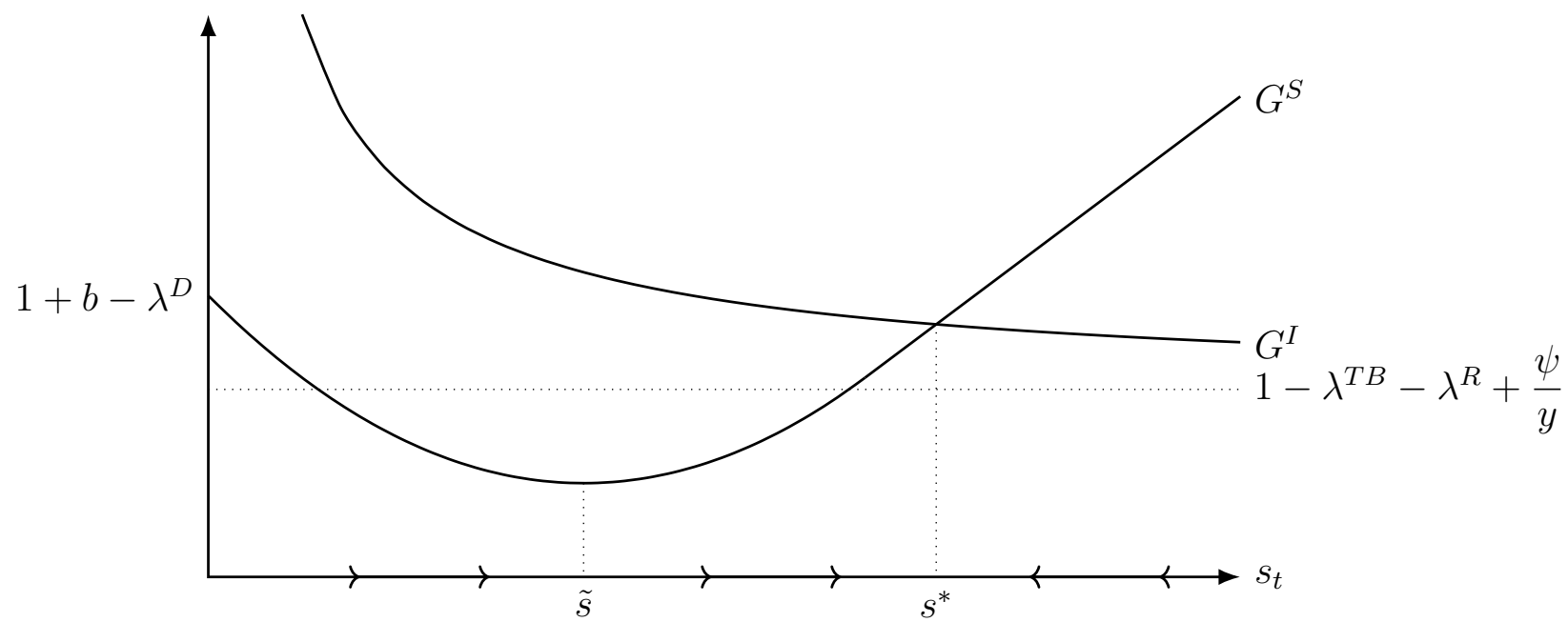

Notes: This figure depicts equations (1) and (2) from the Online Appendix.

Figure A17: Balancing figure
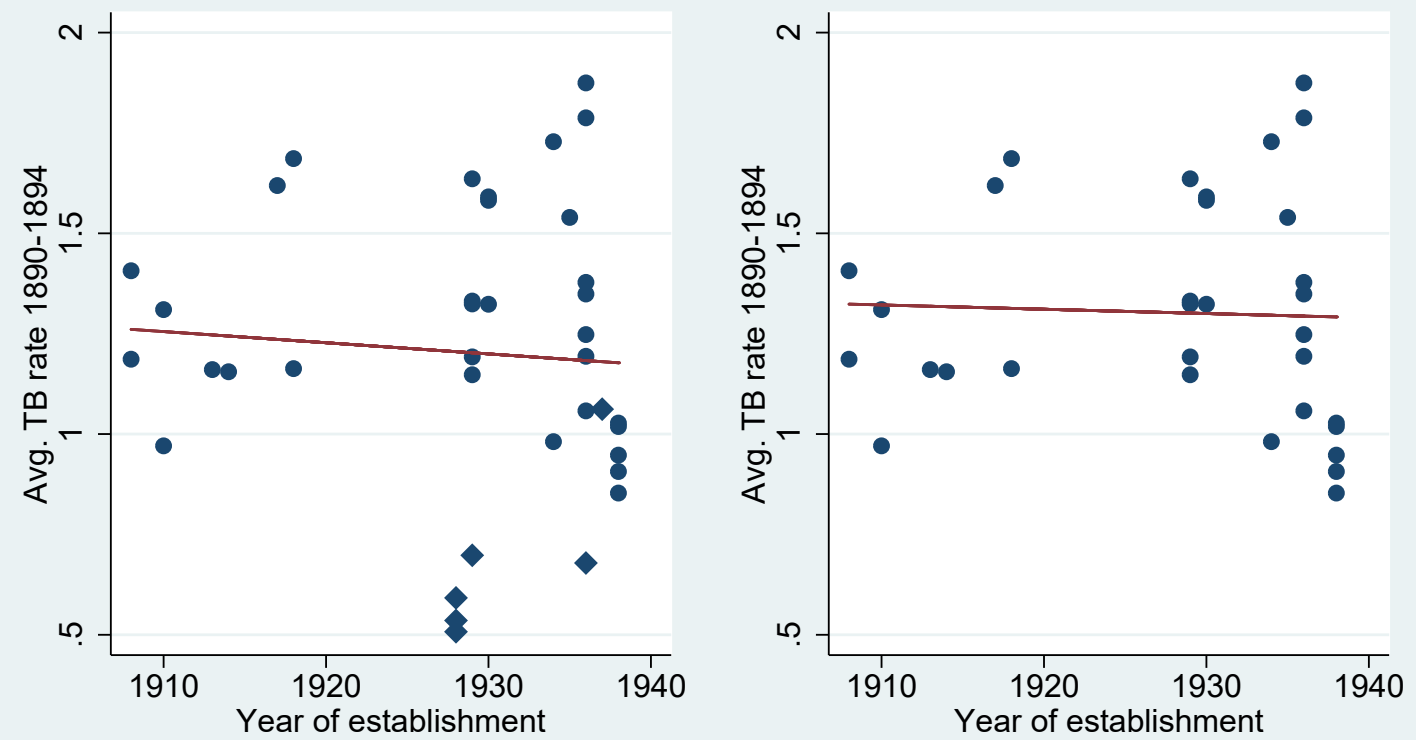

- Cities in balanced panel Cities added to the sample later

Notes: Relationship between the initial TB death rate and the year of establishment of a TB dispensary. 
Figure A18: Event-study estimates of impact on accidents and suicides before and after TB dispensary

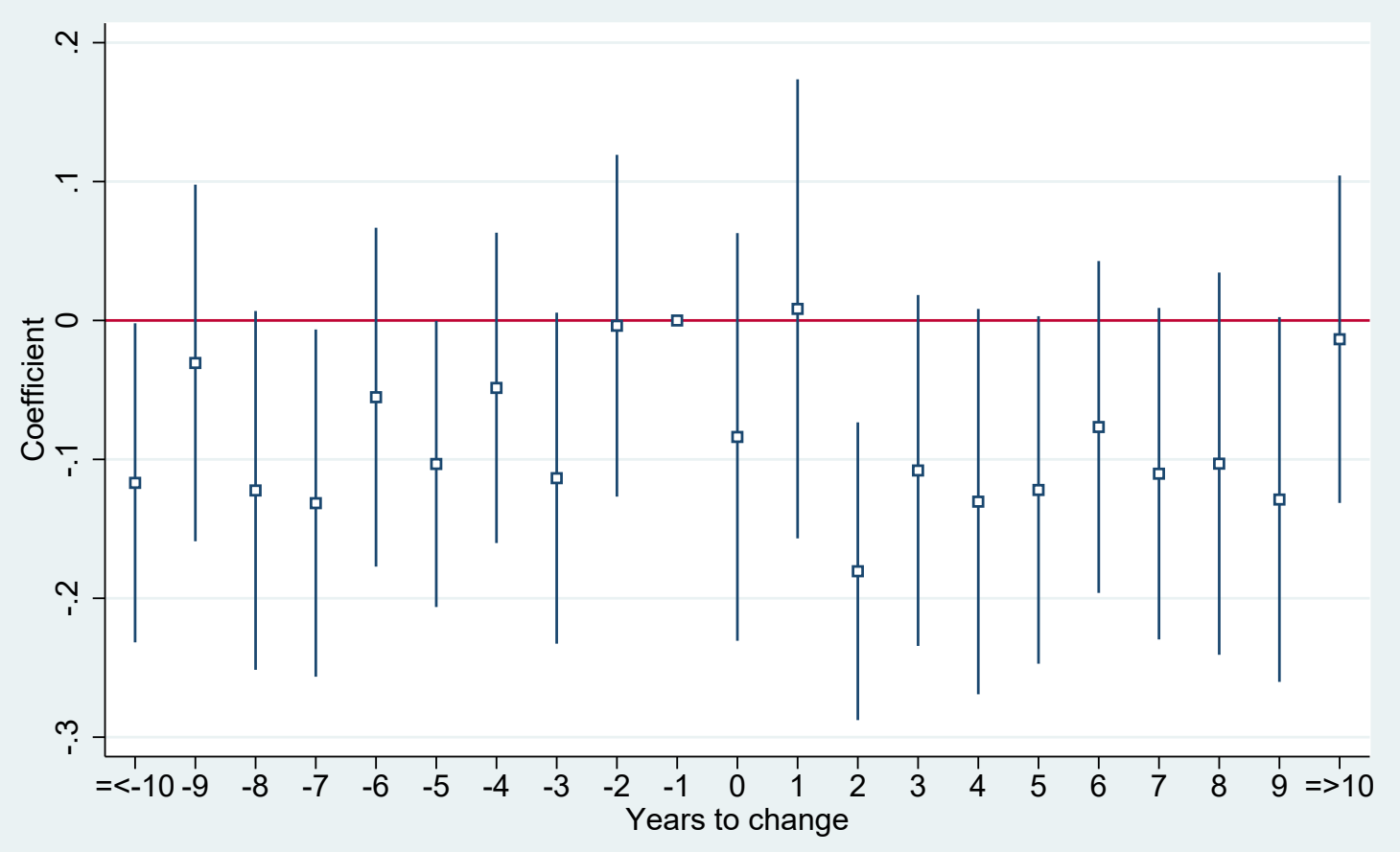

Notes: The graph shows the $\hat{\beta}_{j}$ coefficients and their 95 percent confidence interval from estimating equations (3) and (4) with the accident and suicide (including homicides from 1931) mortality per 1,000 people as the dependent variable and year $\tau-1$ as the baseline, in the year before the intervention. A table with the estimated coefficients is available upon request. 
Figure A19: Event-study estimates of impact on cancer before and after TB dispensary

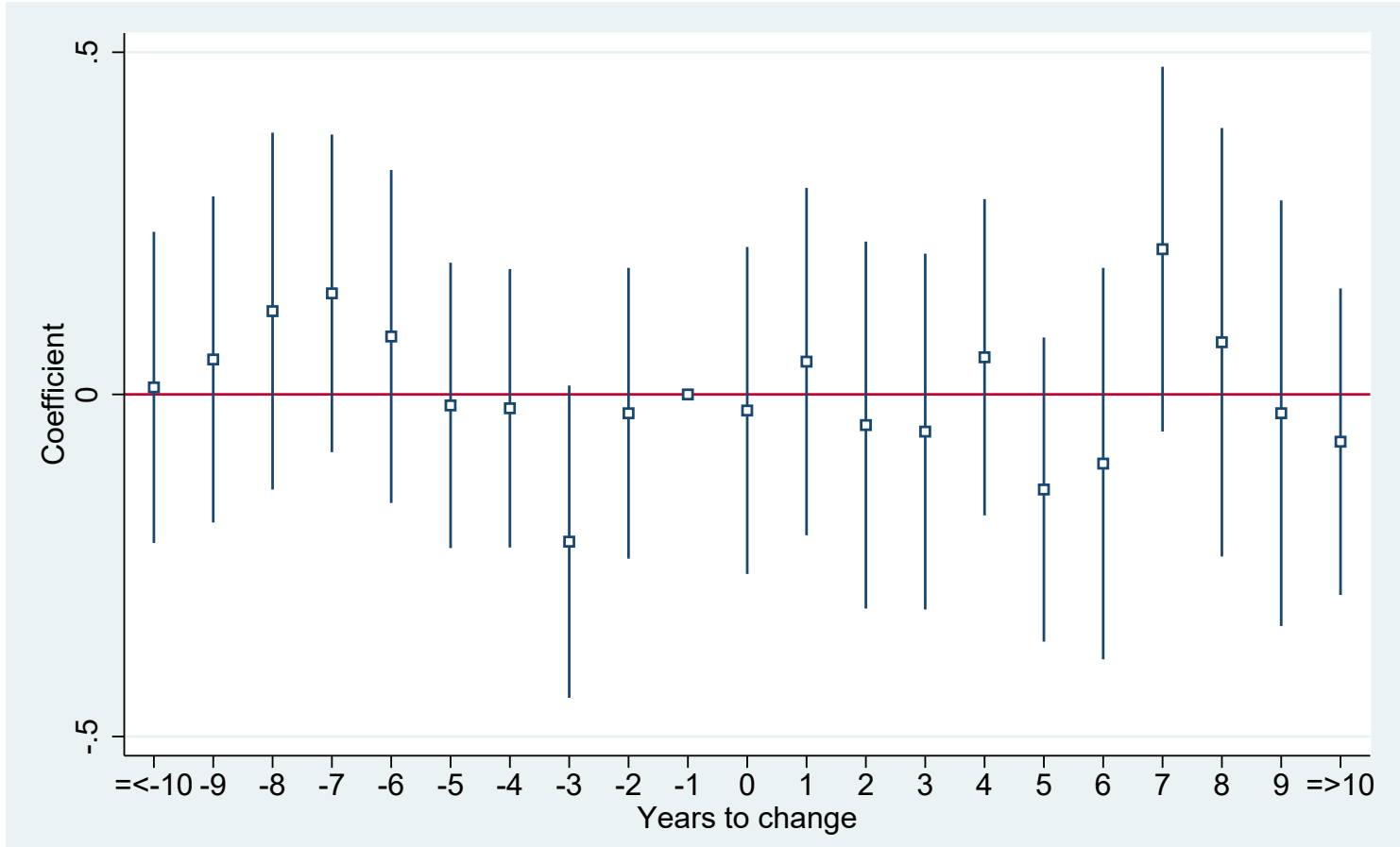

Notes: The graph shows the $\hat{\beta}_{j}$ coefficients and their 95 percent confidence interval from estimating equations (3) and (4) with the cancer mortality per 1,000 people as the dependent variable and year $\tau-1$ as the baseline, in the year before the intervention. A table with the estimated coefficients is available upon request. 
Figure A20: Event-study estimates of impact on diphtheria before and after TB dispensary

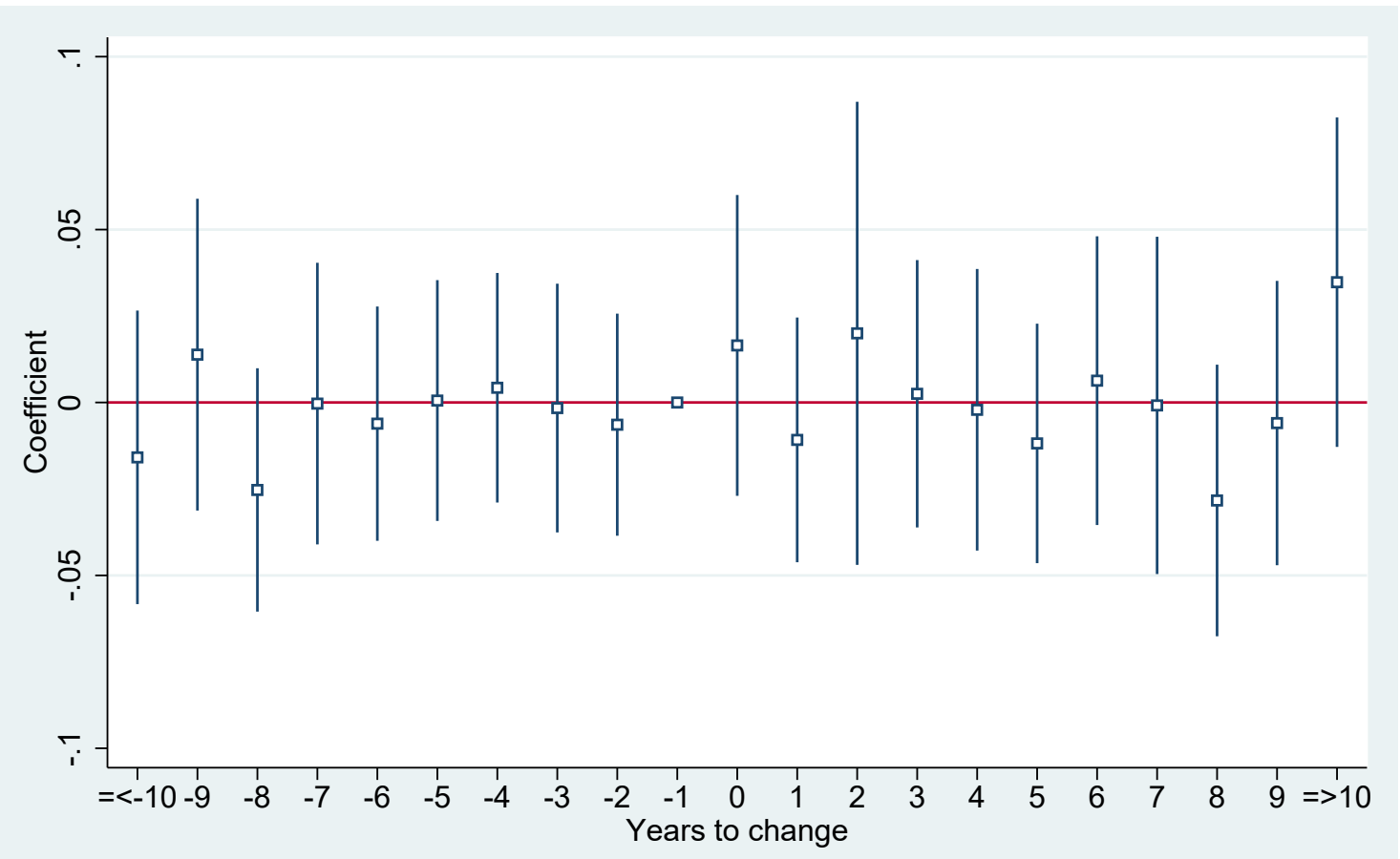

Notes: The graph shows the $\hat{\beta}_{j}$ coefficients and their 95 percent confidence interval from estimating equations (3) and (4) with the diphtheria mortality per 1,000 people as the dependent variable and year $\tau-1$ as the baseline, in the year before the intervention. A table with the estimated coefficients is available upon request. 
Figure A21: Event-study estimates of impact on influenza before and after TB dispensary

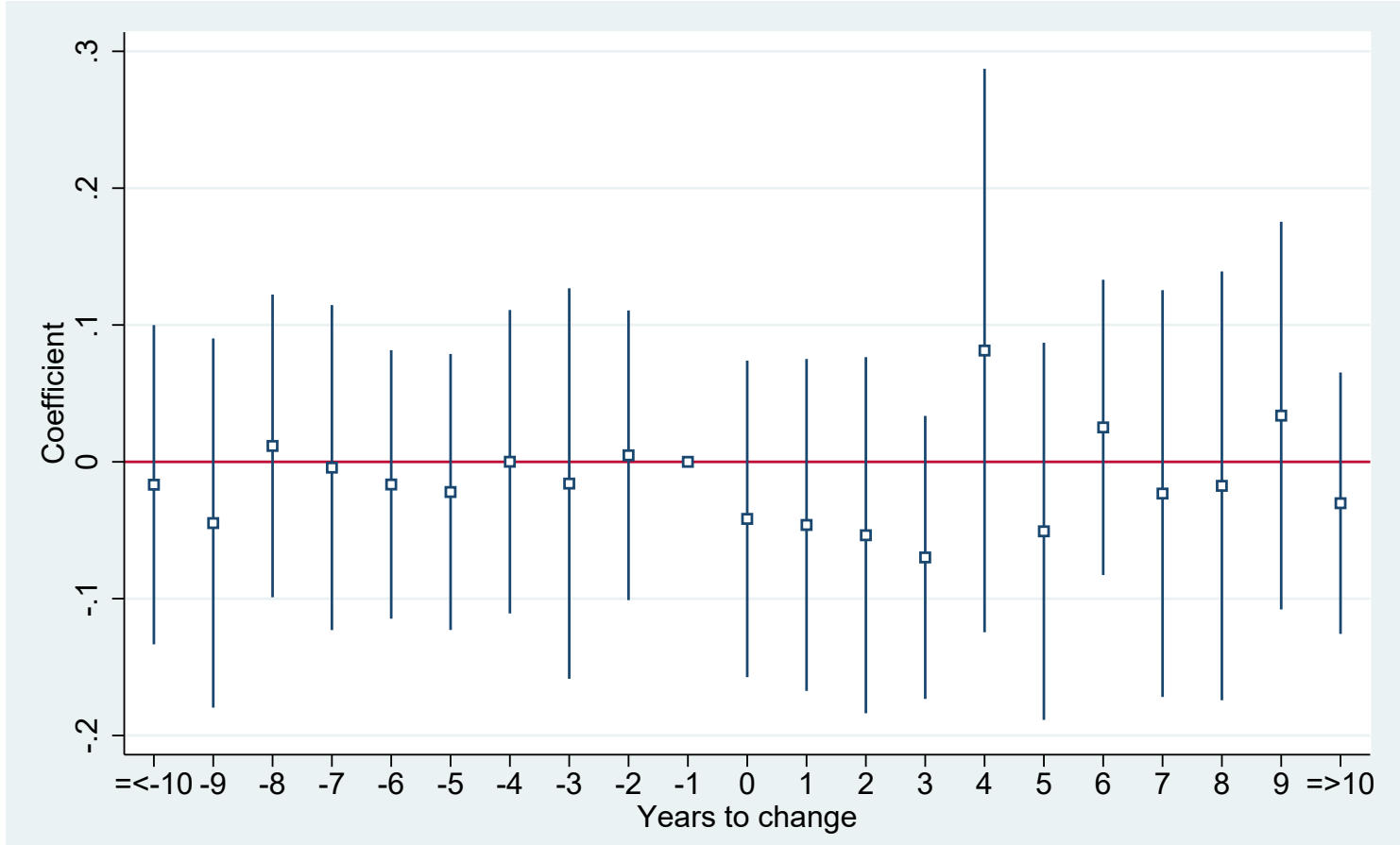

Notes: The graph shows the $\hat{\beta}_{j}$ coefficients and their 95 percent confidence interval from estimating equations (3) and (4) with the influenza mortality per 1,000 people as the dependent variable and year $\tau-1$ as the baseline, in the year before the intervention. A table with the estimated coefficients is available upon request. 
Figure A22: Event-study estimates of impact on pneumonia before and after TB dispensary

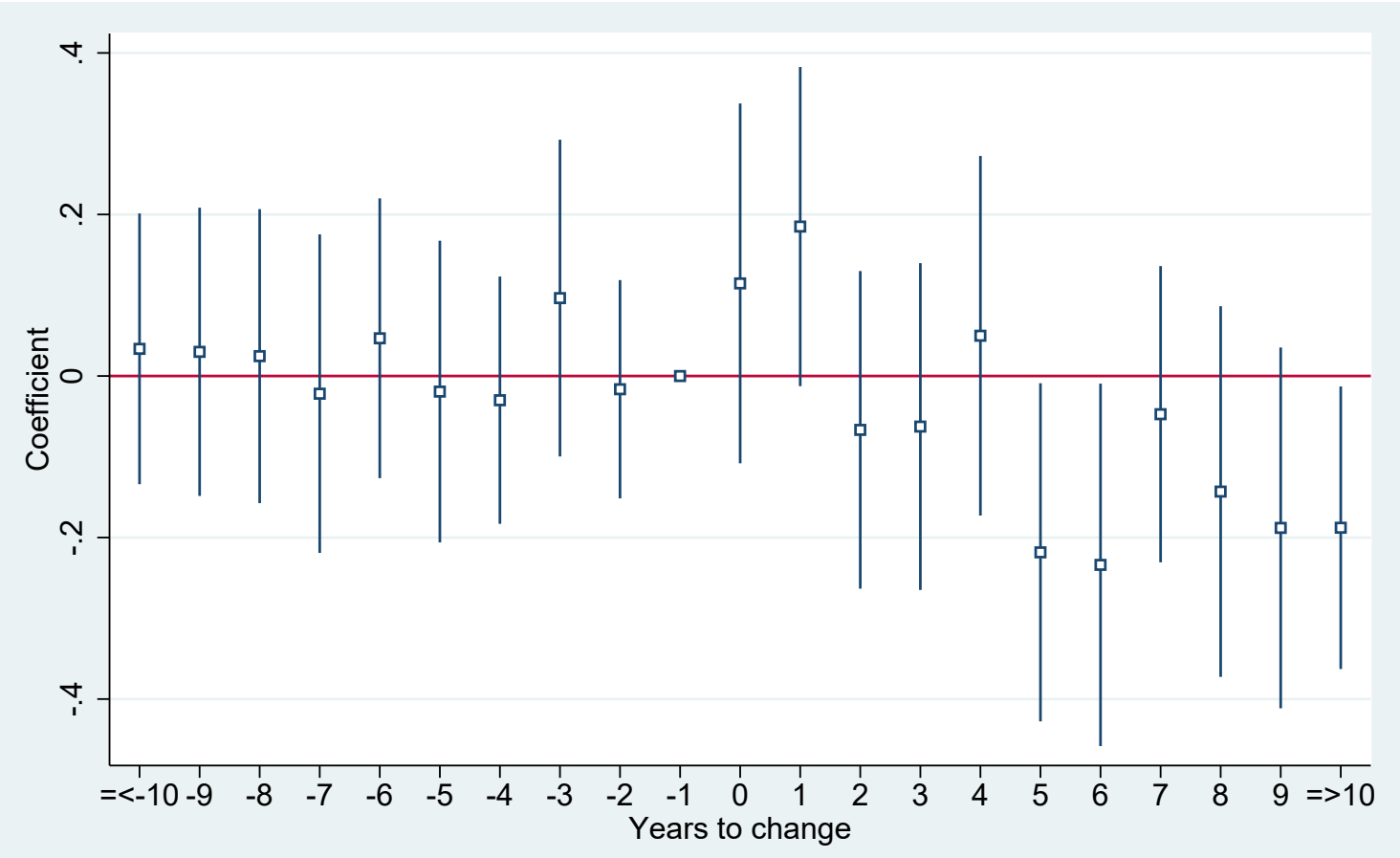

Notes: The graph shows the $\hat{\beta}_{j}$ coefficients and their 95 percent confidence interval from estimating equations (3) and (4) with the pneumonia mortality per 1,000 people as the dependent variable and year $\tau-1$ as the baseline, in the year before the intervention. A table with the estimated coefficients is available upon request. 
Figure A23: Event-study estimates of impact on polio before and after TB dispensary

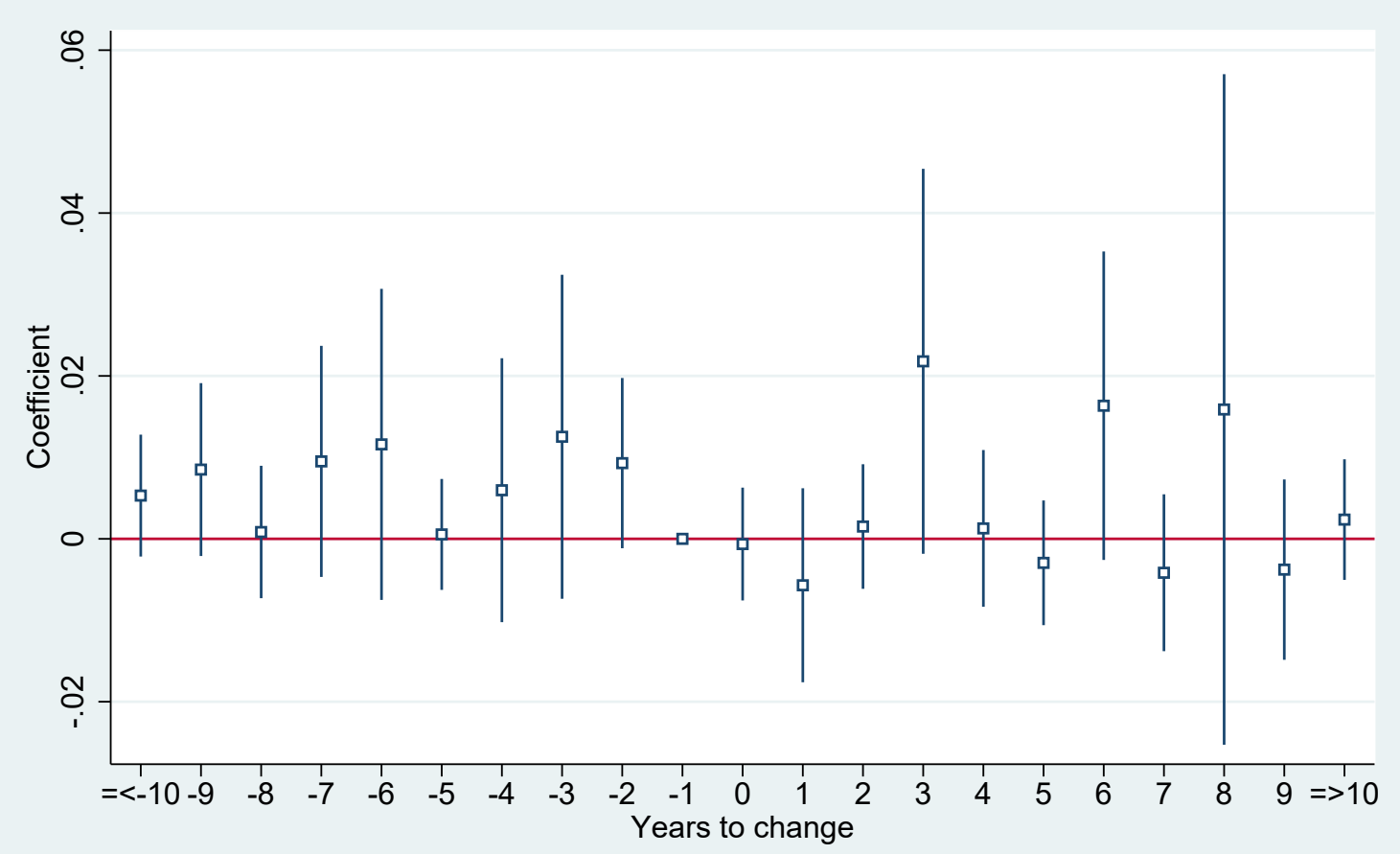

Notes: The graph shows the $\hat{\beta}_{j}$ coefficients and their 95 percent confidence interval from estimating equations (3) and (4) with the polio mortality per 1,000 people as the dependent variable and year $\tau-1$ as the baseline, in the year before the intervention. A table with the estimated coefficients is available upon request. 
Figure A24: Event-study estimates of impact on scarlet fever before and after TB dispensary

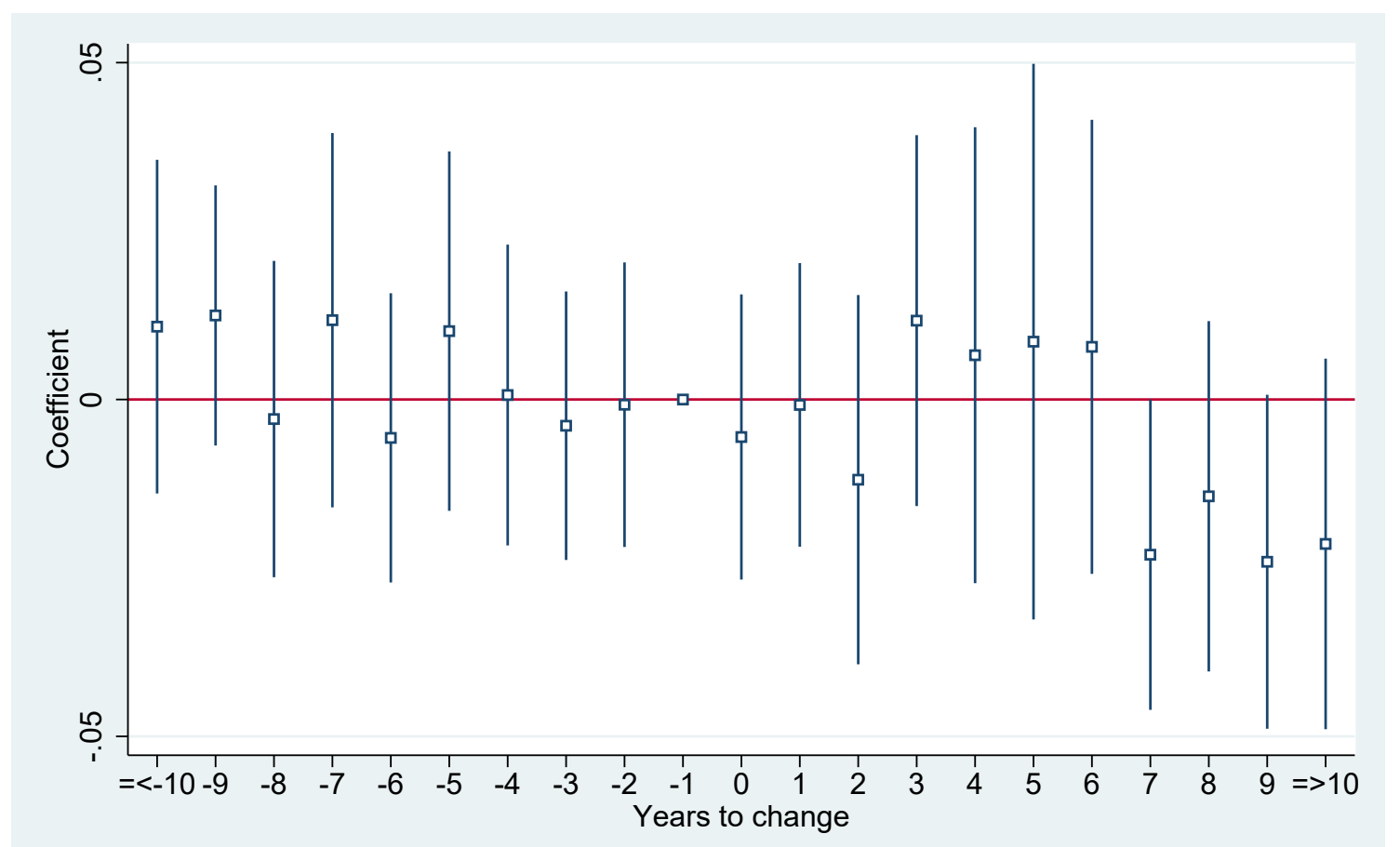

Notes: The graph shows the $\hat{\beta}_{j}$ coefficients and their 95 percent confidence interval from estimating equations (3) and (4) with the scarlet fever mortality per 1,000 people as the dependent variable and year $\tau-1$ as the baseline, in the year before the intervention. A table with the estimated coefficients is available upon request. 
Table A1: Data explanations

\begin{tabular}{|c|c|}
\hline Variable: & Explanation and source: \\
\hline TB rate: & $\begin{array}{l}\text { Number of deaths from any form of tuberculosis per 1,000 people. Source: Cause of } \\
\text { Death Statistics (1890-1950). }\end{array}$ \\
\hline Pulmonary rate: & $\begin{array}{l}\text { Number of deaths from pulmonary tuberculosis per } 1,000 \text { people. Source: Cause of } \\
\text { Death Statistics (1890-1950). }\end{array}$ \\
\hline Birth rate: & Number of live births per 1,000 people (reference). \\
\hline Death rate: & $\begin{array}{l}\text { Number of deaths excluding stillbirths per } 1,000 \text { people, as stillbirths are only available } \\
\text { from 1901. Source: Cause of Death Statistics }(1890-1950) \text {. }\end{array}$ \\
\hline Child mortality rate: & $\begin{array}{l}\text { Number of deaths among } 0-5 \text {-year-olds per } 1,000 \text { live births from } 1901 \text { to } 1936 \text { and } \\
\text { number of deaths among 0-4-year-olds per 1,000 live births from } 1937 \text { to } 1950 \text {. Source: } \\
\text { Cause of Death Statistics (1901-1950). }\end{array}$ \\
\hline Cancer rate: & $\begin{array}{l}\text { Number of deaths from any form of cancer per 1,000 people. Source: Cause of Death } \\
\text { Statistics (1890-1950). }\end{array}$ \\
\hline Influenza rate: & $\begin{array}{l}\text { Number of deaths from influenza per 1,000 people. Source: Cause of Death Statistics } \\
(1890-1950) \text {. }\end{array}$ \\
\hline Pneumonia rate: & $\begin{array}{l}\text { Number of deaths from any form of pneumonia per } 1,000 \text { people. Source: Cause of } \\
\text { Death Statistics }(1890-1950) \text {. }\end{array}$ \\
\hline $\begin{array}{l}\text { Accident and suicide } \\
\text { rate: }\end{array}$ & $\begin{array}{l}\text { Number of deaths from accidents and suicides, including homicides from 1931, per 1,000 } \\
\text { people. Source: Cause of Death Statistics (1890-1950). }\end{array}$ \\
\hline Scarlet fever rate: & $\begin{array}{l}\text { Number of deaths from scarlet fever per 1,000 people. Source: Cause of Death Statistics } \\
(1890-1950) \text {. }\end{array}$ \\
\hline Diphtheria rate: & $\begin{array}{l}\text { Number of deaths from diphtheria per 1,000 people. Source: Cause of Death Statistics } \\
(1890-1950) \text {. }\end{array}$ \\
\hline Polio rate: & $\begin{array}{l}\text { Number of deaths from polio per } 1,000 \text { people. Source: Cause of Death Statistics } \\
(1890-1950) \text {. }\end{array}$ \\
\hline Population: & Number of inhabitants. Source: Cause of Death Statistics (1890-1950). \\
\hline Population density: & $\begin{array}{l}\text { Number of inhabitants in } 1890 \text { per } 1890 \text { acreage of the city. Source: Cause of Death } \\
\text { Statistics (1890) and DigDag. }\end{array}$ \\
\hline $\mathrm{TB} \mathrm{d}$ & $\begin{array}{l}\text { The presence of either a main tuberculosis dispensary or a branch dispensary. Source: } \\
\text { Medical Report (1890-1950). }\end{array}$ \\
\hline Main dispensary: & The presence of a main tuberculosis dispensary. Source: Medical Report (1890-1950). \\
\hline Branch dispensary: & The presence of a branch tuberculosis dispensary. Source: Medical Report (1890-1950). \\
\hline TB hospital: & The presence of a TB hospital. Source: Medical Report (1890-1950). \\
\hline TB hospital patients: & Number of patients at a TB hospital. Source: Medical Report (1908-1939). \\
\hline Sanatorium: & The presence of a sanatorium. Source: Medical Report (1890-1950). \\
\hline Sanatoria patients: & Number of patients at a sanatorium. Source: Medical Report (1901-1939). \\
\hline Income: & $\begin{array}{l}\text { Total income of the inhabitants liable to pay taxes per capita. Source: Statistiske } \\
\text { meddelelser (1905-1940). }\end{array}$ \\
\hline Gini: & $\begin{array}{l}\text { The Gini coefficient for the inhabitants liable to pay taxes calculated using the lowest } \\
\text { point in the intervals of the income distribution. Source: Statistiske meddelelser (1919, } \\
\text { 1922). }\end{array}$ \\
\hline Taxpayers: & Number of inhabitants liable to pay taxes. Source: Statistiske meddelelser (1905-1940). \\
\hline Waterworks: & The presence of waterworks. Source: Medical Report (1890-1950). \\
\hline Housing Association: & $\begin{array}{l}\text { The presence of a housing association. Source: City level archives and various other } \\
\text { local sources. }\end{array}$ \\
\hline
\end{tabular}

Notes: This table describes the main variables used in the analysis.

DigDag is a geographic database of Denmark's historic administrative division, see http://www.digdag.dk/. 
Table A2: Number of TB dispensaries and TB mortality over time

\begin{tabular}{lcccccc}
\hline \hline & & & \multicolumn{4}{c}{ TB rate } \\
\cline { 4 - 7 } Period & No. Cities & No. TB dispensaries & Mean & Std. dev. & Min & Max \\
\hline $1890-94$ & 74 & 0 & 2.613 & 1.224 & 0.000 & 7.123 \\
$1895-99$ & 75 & 0 & 2.139 & 1.063 & 0.000 & 5.556 \\
$1900-04$ & 75 & 0 & 1.948 & 0.976 & 0.000 & 6.667 \\
$1905-09$ & 75 & 2 & 1.546 & 0.894 & 0.000 & 5.549 \\
$1910-14$ & 76 & 6 & 1.275 & 0.721 & 0.000 & 4.502 \\
$1915-19$ & 77 & 9 & 1.120 & 0.765 & 0.000 & 5.490 \\
$1920-24$ & 87 & 9 & 0.939 & 0.581 & 0.000 & 3.243 \\
$1925-29$ & 87 & 18 & 0.733 & 0.507 & 0.000 & 3.077 \\
$1930-34$ & 87 & 23 & 0.625 & 0.511 & 0.000 & 5.484 \\
$1935-39$ & 87 & 38 & 0.453 & 0.410 & 0.000 & 3.636 \\
$1940-46$ & 87 & 71 & 0.330 & 0.311 & 0.000 & 2.000 \\
$1890-1939$ & 87 & 38 & 1.305 & 1.036 & 0.000 & 7.123 \\
$1890-1946$ & 87 & 71 & 1.176 & 1.026 & 0.000 & 7.123 \\
\hline
\end{tabular}

Notes: This table shows the number of TB dispensaries over time along with the five-year average TB mortality rate. Source: The Cause of Death Statistics (1890-1950) and the Medical Reports (1890-1950). 
Table A3: Balance tests

A. Comparing adopters versus non-adopters of TB disp.

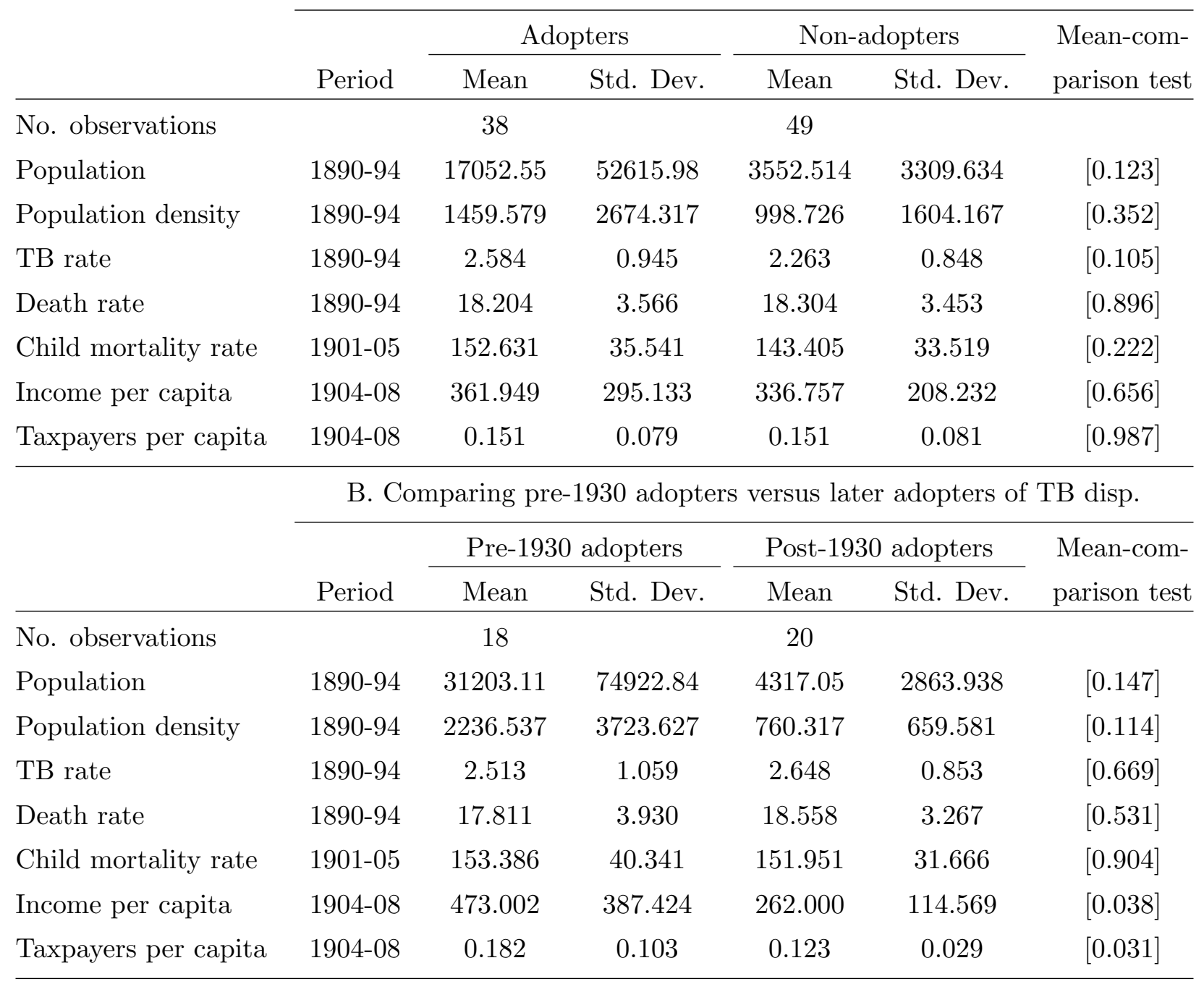

Notes: This table reports balance tests between adopters and non-adopters of TB dispensaries in the period 1890 to 1939 in part A of the table, and balance tests between adopters from 1890 to 1930 and adopters from 1930 and 1939 in part B of the table. The variables compared are the mean of the 1890-1894 population, the population density as the mean of the 1890-1894 population divided by the 1890 acreage of the city in square kilometers, the mean of the 1890-1894 TB death rate, the mean of the 1890-94 death rate, the mean of the 1901-1905 child moratlity rate, the mean of the 1904-1908 income per capita, and the mean of the 1904-1908 taxpayers per capita. For cities included in the data set later, we use the mean of the first five years available. The acreage of the previously German cities are of 1920. In brackets are shown p-values from a mean-comparision Welch's t-test. 
Table A4: Effect of TB dispensaries on the TB death ratio and TB in level

\begin{tabular}{|c|c|c|c|c|c|c|c|}
\hline \multirow[t]{2}{*}{ Dep. variable: } & TB ratio & $\log (\mathrm{TB}$ ratio $)$ & TB ratio & TB ratio & Pulmonary ratio & TB level & Disease level \\
\hline & (1) & $(2)$ & $(3)$ & (4) & $(5)$ & (6) & (7) \\
\hline TB dispensary ${ }_{c, t}$ & $\begin{array}{c}-10.711^{* *} \\
(4.572)\end{array}$ & $\begin{array}{c}-0.125^{* *} \\
(0.049)\end{array}$ & $\begin{array}{c}-10.289^{* *} \\
(4.168)\end{array}$ & $\begin{array}{c}-9.407^{* *} \\
(3.625)\end{array}$ & $\begin{array}{c}-9.494^{* *} \\
(3.695)\end{array}$ & $\begin{array}{c}-16.263^{*} \\
(8.381)\end{array}$ & $\begin{array}{c}-10.914^{* * *} \\
(4.038)\end{array}$ \\
\hline $\mathrm{TB} \operatorname{ratio}_{c, t-1}$ & & & $\begin{array}{c}0.081^{* * *} \\
(0.025)\end{array}$ & $\begin{array}{c}0.067^{* * *} \\
(0.023)\end{array}$ & & & \\
\hline TB ratio $_{c, t-2}$ & & & & $\begin{array}{l}0.043^{* *} \\
(0.019)\end{array}$ & & & \\
\hline TB $\operatorname{ratio}_{c, t-3}$ & & & & $\begin{array}{c}0.069^{* * *} \\
(0.020)\end{array}$ & & & \\
\hline TB ratio ${ }_{c, t-4}$ & & & & $\begin{array}{l}0.048^{* *} \\
(0.019)\end{array}$ & & & \\
\hline TB ratio $_{c, t-5}$ & & & & $\begin{array}{c}0.033^{*} \\
(0.019)\end{array}$ & & & \\
\hline Avg. TB & 89.239 & 4.398 & 88.164 & 84.215 & 59.350 & 19.962 & 18.053 \\
\hline Long run effect & & & $-11.199^{* *}$ & $-12.720^{* *}$ & & & \\
\hline City FE & Yes & Yes & Yes & Yes & Yes & Yes & Yes \\
\hline Year FE & Yes & Yes & Yes & Yes & Yes & Yes & Yes \\
\hline Disease FE & No & No & No & No & No & No & Yes \\
\hline City FE $\times$ Year FE & No & No & No & No & No & No & Yes \\
\hline Disease FE $\times$ Year FE & No & No & No & No & No & No & Yes \\
\hline Disease FE $\times$ City FE & No & No & No & No & No & No & Yes \\
\hline Time period & 1890-1939 & $1890-1939$ & 1891-1939 & $1895-1939$ & 1901-1939 & 1890-1939 & 1901-1939 \\
\hline Observations & 3,981 & 3,662 & 3,894 & 3,546 & 3,165 & 3,981 & 25,320 \\
\hline R-squared & 0.45 & 0.49 & 0.45 & 0.45 & 0.35 & 0.94 & 0.92 \\
\hline Cities & 87 & 87 & 87 & 87 & 87 & 87 & 87 \\
\hline
\end{tabular}

Notes: The table reports least squares estimates. In columns (1), (3), and (4) the left-hand-side variable is the TB mortality per 1,000 death, in column (2) the log is taken of this variable, in column (5) the left-hand-side variable is the pulmonary TB mortality per 1,000 death, in column (6) the left-hand-side variable is TB deaths, and in column (7) the left-hand-side variable is the stacked causes of death from TB, cancer, influenza, pneumonia, accidents and suicides (including homicides from 1931), scarlet fever, diphtheria, and polio in levels. TB dispensary ${ }_{c, t}$ is an indicator variable equal to one after the introduction of a TB dispensary multiplied by an indicator equal to one if the disease on the left-hand-side is TB, and TB rate ${ }_{c, t-i}$ is the $i$ year lagged dependent variable. Long run effect is the steady-state value of the estimated model in column (3), and (4). All regressions includes city and year fixed effects, and addtionally column (7) includes disease, disease-by-year, disease-by-city, and city-by-year fixed effects. Robust standard errors clustered at the city level are in parentheses.

${ }^{*},{ }^{* *}$ and ${ }^{* * *}$ determine significance levels of 10 percent, 5 percent and 1 percent, respectively. 
Table A5: Event studies of the impact of a TB dispensary

\begin{tabular}{|c|c|c|c|c|c|}
\hline \multirow[t]{2}{*}{ Dep. variable: } & TB rate & Disease & $\operatorname{Dispensary}_{c, t+4}^{\tau+4}$ & \multirow{2}{*}{$\begin{array}{c}-0.278^{* *} \\
(0.138)\end{array}$} & \multirow{2}{*}{$\begin{array}{c}-0.283^{* *} \\
(0.128)\end{array}$} \\
\hline & (1) & $(2)$ & \multirow{2}{*}{ Dispensary $_{c, t+5}^{\tau+5}$} & & \\
\hline \multirow[t]{2}{*}{$\operatorname{Dispensary}_{c, t-10}^{\tau-10}$} & 0.102 & 0.099 & & $(0.105)$ & $(0.105)$ \\
\hline & $(0.105)$ & $(0.104)$ & \multirow{2}{*}{$\operatorname{Dispensary}_{c, t+6}^{\tau+6}$} & $-0.300^{* * *}$ & $-0.244^{* *}$ \\
\hline \multirow[t]{2}{*}{$\operatorname{Dispensary}_{c, t-9}^{\tau-9}$} & 0.015 & 0.008 & & $(0.101)$ & $(0.103)$ \\
\hline & $(0.092)$ & $(0.101)$ & \multirow{2}{*}{$\operatorname{Dispensary}_{c, t+7}^{\tau+7}$} & $-0.291^{* *}$ & $-0.285^{* *}$ \\
\hline \multirow[t]{2}{*}{ Dispensary $_{c, t-8}^{\tau-8}$} & 0.028 & 0.027 & & $(0.134)$ & $(0.133)$ \\
\hline & $(0.085)$ & $(0.092)$ & \multirow[t]{2}{*}{ Dispensary $_{c, t+8}^{\tau+8}$} & $-0.254^{* *}$ & $-0.217^{*}$ \\
\hline \multirow[t]{2}{*}{ Dispensary $_{c, t-7}^{\tau-7}$} & 0.074 & 0.073 & & $(0.116)$ & $(0.118)$ \\
\hline & $(0.086)$ & $(0.090)$ & \multirow[t]{2}{*}{ Dispensary $_{c, t+9}^{\tau+9}$} & $-0.320^{* * *}$ & $-0.265^{* *}$ \\
\hline \multirow[t]{2}{*}{ Dispensary $_{c, t-6}^{\tau-6}$} & 0.074 & 0.073 & & $(0.110)$ & $(0.113)$ \\
\hline & $(0.086)$ & $(0.090)$ & \multirow{2}{*}{ Dispensary $_{c, t+10}^{\tau+10}$} & $-0.249^{* *}$ & $-0.225^{* *}$ \\
\hline Dispensary $_{c, t-5}^{\tau-5}$ & $\begin{array}{l}-0.143 \\
(0.090)\end{array}$ & $\begin{array}{l}-0.121 \\
(0.099)\end{array}$ & & $(0.111)$ & $(0.108)$ \\
\hline \multirow{2}{*}{ Dispensary $_{c, t-4}^{\tau-4}$} & 0.001 & 0.020 & City & Yes & Yes \\
\hline & $(0.084)$ & $(0.087)$ & Yea & $Y \epsilon$ & Yes \\
\hline \multirow[t]{2}{*}{$\operatorname{Dispensary}_{c, t-3}^{\tau-3}$} & 0.012 & 0.049 & Disease FE & No & Yes \\
\hline & $(0.085)$ & $(0.083)$ & Disease FE $\times$ & & $\mathrm{Y}$ \\
\hline \multirow{2}{*}{ Dispensary $_{c, t-2}^{\tau-2}$} & -0.024 & -0.016 & Year FE & No & Yes \\
\hline & $(0.08$ & $(0.089)$ & $\begin{array}{l}\text { Disease FE } \times \\
\text { City FE }\end{array}$ & No & Yes \\
\hline $\operatorname{Dispensary}_{c, t}^{\tau}$ & -0.150 & -0.147 & City FE $\times$ & & \\
\hline \multirow[t]{2}{*}{$\operatorname{Dispensary}_{c, t+1}^{\tau+1}$} & $\begin{array}{l}(0.104) \\
-0.117\end{array}$ & $\begin{array}{l}(0.110) \\
-0.142^{*}\end{array}$ & Year FE & No & Yes \\
\hline & $(0.074)$ & $(0.079)$ & Tir & 1890-1939 & 1901-1939 \\
\hline \multirow[t]{2}{*}{ Dispensary $_{c, t+2}^{\tau+2}$} & $-0.225^{* *}$ & -0.170 & Observations & 3,981 & 25,320 \\
\hline & $(0.106)$ & $(0.111)$ & R-squared & 0.54 & 0.70 \\
\hline Dispensary $_{c, t+3}^{\tau+3}$ & $-0.189^{* *}$ & -0.144 & Cities & 87 & 87 \\
\hline
\end{tabular}

Notes: The table reports least squares estimates. In column (1) the left-hand-side variable is the TB mortality per 1,000 people, and in column (2) the left-hand-side variable is the stacked causes of death from TB, cancer, influenza, pneumonia, accidents and suicides (including homicides from 1931), scarlet fever, diphtheria, and polio per 1,000 people. All regressions includes city and year fixed effects, and addtionally column (2) includes disease, diseaseby-year, disease-by-city, and city-by-year fixed effects. TB dispensary $\tau_{c, t+j}^{\tau+j}$ were $T=\{-10, \ldots,-2,0, \ldots, 10\}$ is an indicator equal to one when $t=\tau+j$ where $\tau$ marks the period of introduction of a TB dispensary. All indicators are multiplied by an indicator equal to one if the disease on the left-hand side is TB. Robust standard errors clustered at the city level are in parentheses.

${ }^{*},{ }^{* *}$ and ${ }^{* * *}$ determine significance levels of 10 percent, 5 percent and 1 percent, respectively. 
Table A6: Effect of TB dispensaries using city-by-year-by-disease data with alternative disease panels

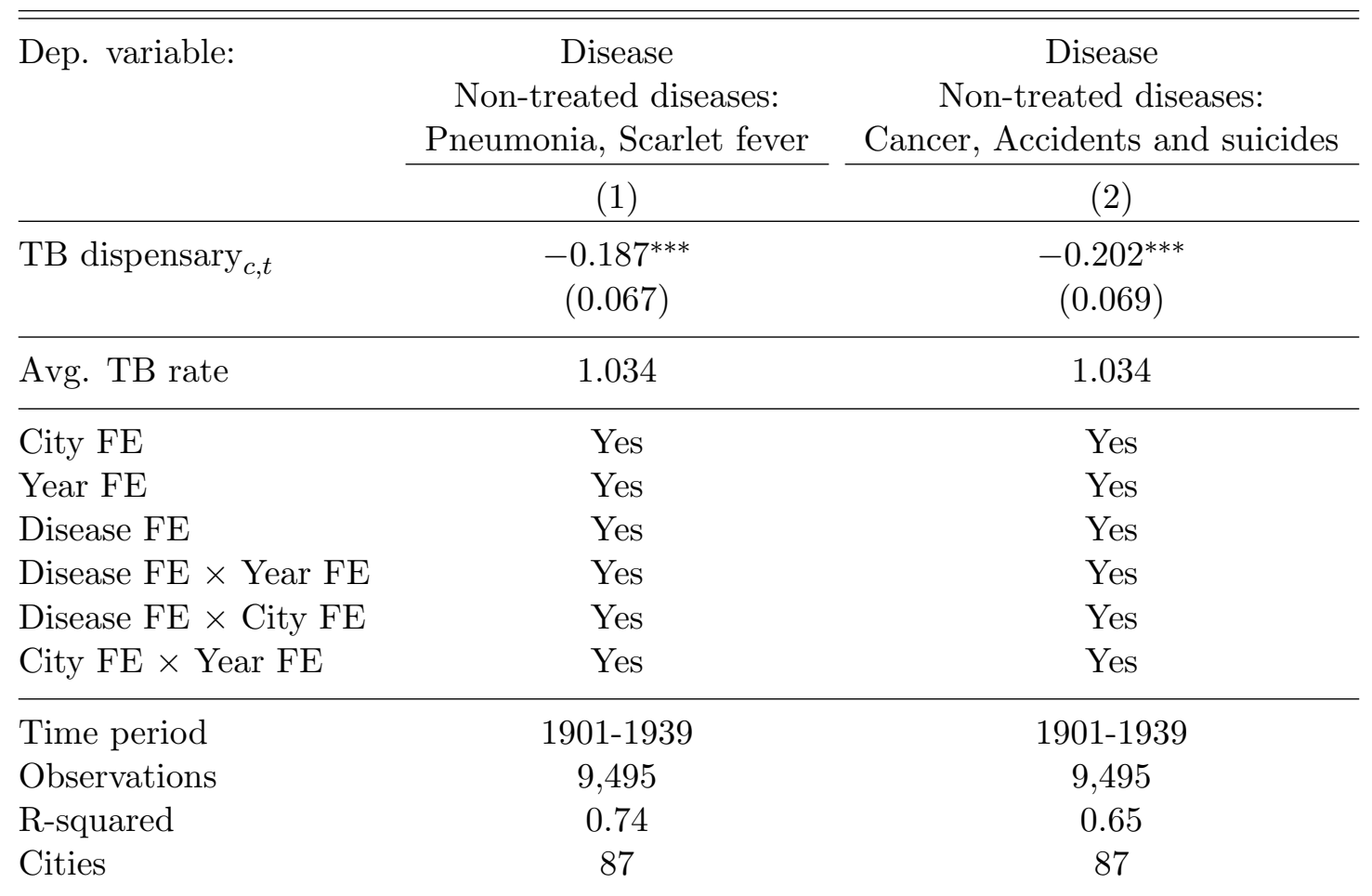

Notes: The table reports least squares estimates. In column (1) the left-hand-side variable is the stacked causes of death from TB, pneumonia, and scarlet fever per 1,000 people. In column (2) the left-hand-side variable is the stacked causes of death from TB, cancer, and accidents and suicides (including homicides from 1931) per 1,000 people. All regressions include city, year, disease, disease-by-year, disease-by-city, and city-by-year fixed effects. TB dispensary ${ }_{c, t}$ is an indicator variable equal to one after the introduction of a TB dispensary multiplied by an indicator equal to one if the disease on the left-hand side is TB. Robust standard errors clustered at the city level are in parentheses.

${ }^{*},{ }^{* *}$ and ${ }^{* * *}$ determine significance levels of 10 percent, 5 percent and 1 percent, respectively. 
Table A7: Effect of TB dispensaries; alternative treatment measures

\begin{tabular}{|c|c|c|c|c|}
\hline \multirow[t]{2}{*}{ Dep. variable: } & TB rate & Disease & TB rate & Disease \\
\hline & $(1)$ & $(2)$ & $(3)$ & $(4)$ \\
\hline $\mathrm{TB}_{\text {dispensary }}{ }_{c, t} \times\left(t+1-j_{c}\right)$ & $\begin{array}{c}-0.013^{* *} \\
(0.005)\end{array}$ & $\begin{array}{c}-0.009^{*} \\
(0.005)\end{array}$ & & \\
\hline TB dispensary det $\times \mathrm{TB}$ rate $_{c, j}$ & & & $\begin{array}{c}-0.164^{* *} \\
(0.065)\end{array}$ & $\begin{array}{c}-0.153^{* * *} \\
(0.054)\end{array}$ \\
\hline Avg. TB rate & 1.306 & 1.034 & 1.306 & 1.034 \\
\hline City FE & Yes & Yes & Yes & Yes \\
\hline Year FE & Yes & Yes & Yes & Yes \\
\hline Disease FE & No & Yes & No & Yes \\
\hline City $\mathrm{FE} \times$ Year FE & No & Yes & No & Yes \\
\hline Disease $\mathrm{FE} \times$ Year FE & No & Yes & No & Yes \\
\hline Disease FE $\times$ City FE & No & Yes & No & Yes \\
\hline Time period & 1890-1939 & 1901-1939 & 1890-1939 & 1901-1939 \\
\hline Observations & 3,981 & 25,320 & 3,981 & 25,320 \\
\hline R-squared & 0.53 & 0.70 & 0.53 & 0.70 \\
\hline Cities & 87 & 87 & 87 & 87 \\
\hline
\end{tabular}

Notes: The table reports least squares estimates. In columns (1) and (3) the left-hand-side variable is the TB mortality per 1,000 people, and in columns (2) and (4) the left-hand-side variable is the stacked causes of death from TB, cancer, influenza, pneumonia, accidents and suicides (including homicides from 1931), scarlet fever, diphtheria, and polio per 1,000 people. All regressions include city and year fixed effects, and addtionally column (2), and (4) includes disease, disease-by-year, disease-by-city, and city-by-year fixed effects. TB dispensary ${ }_{c, t}$ is an indicator variable equal to one after the introduction of a TB dispensary multiplied by an indicator equal to one if the disease on the left-hand side is $\mathrm{TB}, j_{c}$ is the year of the introduction of a dispensary, $t$ is the year, and TB rate $_{c, j}$ is the average TB rate in the five years before the introduction of a TB dispensary standardized to have a zero mean and standard deviation of one. Robust standard errors clustered at the city level are in parentheses.

${ }^{*},{ }^{* *}$ and ${ }^{* * *}$ determine significance levels of 10 percent, 5 percent and 1 percent, respectively. 
Table A8: Effect of TB dispensaries controlling for early BCG trials

\begin{tabular}{|c|c|c|c|c|}
\hline \multirow[t]{3}{*}{ Dep. variable: } & $\mathrm{TB}$ rate & Disease & TB rate & Disease \\
\hline & \multicolumn{2}{|c|}{ Excluding Bornholm } & \multicolumn{2}{|c|}{ Panel limited to 1935} \\
\hline & $(1)$ & $(2)$ & $(3)$ & $(4)$ \\
\hline TB dispensary ${ }_{c, t}$ & $\begin{array}{c}-0.230^{* * *} \\
(0.082)\end{array}$ & $\begin{array}{c}-0.194^{* * *} \\
(0.069)\end{array}$ & $\begin{array}{c}-0.236^{* *} \\
(0.090)\end{array}$ & $\begin{array}{c}-0.202^{* *} \\
(0.080)\end{array}$ \\
\hline Avg. TB rate & 1.307 & 1.390 & 1.030 & 1.109 \\
\hline City FE & Yes & Yes & Yes & Yes \\
\hline Year FE & Yes & Yes & Yes & Yes \\
\hline Disease FE & No & Yes & No & Yes \\
\hline City FE $\times$ Year FE & No & Yes & No & Yes \\
\hline Disease $\mathrm{FE} \times$ Year FE & No & Yes & No & Yes \\
\hline Disease FE $\times$ City FE & No & Yes & No & Yes \\
\hline Time period & $1890-1939$ & 1901-1939 & $1890-1935$ & $1901-1935$ \\
\hline Observations & 3,681 & 23,448 & 3,633 & 22,536 \\
\hline R-squared & 0.56 & 0.71 & 0.51 & 0.70 \\
\hline Cities & 81 & 81 & 87 & 87 \\
\hline
\end{tabular}

Notes: The table reports least squares estimates. In columns (1) and (3) the left-hand-side variable is the TB mortality per 1,000 people, and in columns (2) and (4) the left-hand-side variable is the stacked causes of death from TB, cancer, influenza, pneumonia, accidents and suicides (including homicides from 1931), scarlet fever, diphtheria, and polio per 1,000 people. All regressions include city and year fixed effects, and addtionally columns (2) and (4) include disease, disease-by-year, disease-by-city, and city-by-year fixed effects. TB dispensary ${ }_{c, t}$ is an indicator variable equal to one after the introduction of a TB dispensary multiplied by an indicator equal to one if the disease on the left-hand side is TB. In columns (1) and (2) the six cities on the island of Bornholm are excluded from the sample, and in columns (3) and (4) the years 1936 to 1939 are excluded. Robust standard errors clustered at the city level are in parentheses.

${ }^{*},{ }^{* *}$ and ${ }^{* * *}$ determine significance levels of 10 percent, 5 percent and 1 percent, respectively. 
Table A9: Effect of TB dispensaries excluding large cities and early adopters

\begin{tabular}{|c|c|c|c|c|c|c|}
\hline \multirow{3}{*}{ Dep. variable: } & $\mathrm{TB}$ rate & Disease & $\mathrm{TB}$ rate & Disease & $\mathrm{TB}$ rate & Disease \\
\hline & \multicolumn{2}{|c|}{ Excluding Copenhagen } & \multicolumn{2}{|c|}{ Excluding 5 largest cities } & \multicolumn{2}{|c|}{ Excluding pre-1920 adopters } \\
\hline & $(1)$ & $(2)$ & $(3)$ & $(4)$ & $(5)$ & $(6)$ \\
\hline TB dispensary ${ }_{c, t}$ & $\begin{array}{c}-0.259^{* * *} \\
(0.079)\end{array}$ & $\begin{array}{c}-0.216^{* * *} \\
(0.065)\end{array}$ & $\begin{array}{c}-0.301^{* * *} \\
(0.085)\end{array}$ & $\begin{array}{c}-0.246^{* * *} \\
(0.067)\end{array}$ & $\begin{array}{c}-0.315^{* * *} \\
(0.094)\end{array}$ & $\begin{array}{c}-0.260^{* * *} \\
(0.076)\end{array}$ \\
\hline Avg. TB rate & 1.302 & 1.030 & 1.289 & 1.020 & 1.309 & 1.034 \\
\hline City FE & Yes & Yes & Yes & Yes & Yes & Yes \\
\hline Year FE & Yes & Yes & Yes & Yes & Yes & Yes \\
\hline Disease FE & No & Yes & No & Yes & No & Yes \\
\hline Disease $\mathrm{FE} \times$ Year FE & No & Yes & No & Yes & No & Yes \\
\hline Disease FE $\times$ City FE & No & Yes & No & Yes & No & Yes \\
\hline City FE × Year FE & No & Yes & No & Yes & No & Yes \\
\hline Time period & 1890-1939 & 1901-1939 & 1890-1939 & 1901-1939 & 1890-1939 & 1901-1939 \\
\hline Observations & 3,931 & 25,008 & 3,731 & 23,760 & 3,341 & 20,992 \\
\hline R-squared & 0.53 & 0.69 & 0.52 & 0.69 & 0.51 & 0.69 \\
\hline Cities & 86 & 86 & 82 & 82 & 68 & 68 \\
\hline
\end{tabular}

Notes: The table reports least squares estimates. In columns (1), (3), and (5) the left-hand-side variable is the TB mortality per 1,000 people, and in columns (2), (4), and (6) the left-hand-side variable is the stacked causes of death from TB, cancer, influenza, pneumonia, accidents and suicides (including homicides from 1931), scarlet fever, diphtheria, and polio per 1,000 people. All regressions include city and year fixed effects, and addtionally columns (2), (4), and (6) include disease, disease-by-year, disease-by-city, and city-by-year fixed effects. TB dispensary , $_{t}$ is an indicator variable equal to one after the introduction of a TB dispensary multiplied by an indicator equal to one if the disease on the left-hand side is TB. In columns (1) and (2) the city Copenhagen is excluded from the sample, in columns (3) and (4) the five largest cities as of 1890 and 1901 are excluded, and in columns (5) and (6) cities that adopted a TB dispensary before 1920 are excluded. Robust standard errors clustered at the city level are in parentheses.

${ }^{*},{ }^{* *}$ and ${ }^{* * *}$ determine significance levels of 10 percent, 5 percent and 1 percent, respectively. 
Table A10: Effect of TB dispensaries controlling for lagged population, initial population, weighting by city sizes, and linear trend

\begin{tabular}{|c|c|c|c|c|c|c|c|}
\hline \multirow[t]{3}{*}{ Dep. variable: } & TB rate & Disease & $\mathrm{TB}$ rate & Disease & TB rate & Disease & \multirow{2}{*}{$\frac{\text { TB rate }}{\text { OLS }}$} \\
\hline & \multicolumn{4}{|c|}{ OLS } & \multicolumn{2}{|c|}{ WLS } & \\
\hline & $(1)$ & $(2)$ & $(3)$ & (4) & $(5)$ & (6) & $(7)$ \\
\hline TB dispensary ${ }_{c, t}$ & $\begin{array}{c}-0.205^{* * *} \\
(0.074)\end{array}$ & $\begin{array}{c}-0.195^{* * *} \\
(0.064)\end{array}$ & $\begin{array}{c}-0.268^{* * *} \\
(0.081)\end{array}$ & $\begin{array}{c}-0.231^{* * *} \\
(0.063)\end{array}$ & $\begin{array}{c}-0.231^{* * *} \\
(0.075)\end{array}$ & $\begin{array}{c}-0.196^{* * *} \\
(0.065)\end{array}$ & $\begin{array}{l}-0.104 \\
(0.072)\end{array}$ \\
\hline $\log \left(\right.$ Population $\left._{c, t-1}\right)$ & $\begin{array}{c}-0.669^{* * *} \\
(0.225)\end{array}$ & $\begin{array}{l}-0.232 \\
(0.214)\end{array}$ & & & & & \\
\hline Avg. TB rate & 1.280 & 1.013 & 1.306 & 1.034 & 1.306 & 1.034 & 1.306 \\
\hline City FE & Yes & Yes & Yes & Yes & Yes & Yes & Yes \\
\hline Year FE & Yes & Yes & Yes & Yes & Yes & Yes & Yes \\
\hline Disease FE & No & Yes & No & Yes & No & Yes & No \\
\hline City FE $\times$ Year FE & No & Yes & No & Yes & No & Yes & No \\
\hline Disease $\mathrm{FE} \times$ Year FE & No & Yes & No & Yes & No & Yes & No \\
\hline Disease FE $\times$ City FE & No & Yes & No & Yes & No & Yes & No \\
\hline Initial $\log ($ population $) \times$ & & & & & & & \\
\hline Year FE & No & No & Yes & Yes & No & No & No \\
\hline Linear trend $\times$ & & & & & & & \\
\hline City FE & No & No & No & No & No & No & Yes \\
\hline Time period & 1891-1939 & 1902-1939 & 1890-1939 & 1901-1939 & 1890-1939 & 1901-1939 & 1890-1939 \\
\hline Observations & 3,894 & 24,624 & 3,981 & 25,320 & 3,981 & 25,320 & 3,981 \\
\hline R-squared & 0.54 & 0.70 & 0.54 & 0.70 & 0.55 & 0.71 & 0.58 \\
\hline Cities & 87 & 87 & 87 & 87 & 87 & 87 & 87 \\
\hline
\end{tabular}

Notes: The table reports ordinary least squares estimates, except columns (5) and (6) repporting least squares weighted on initial log population. In columns (1), (3), (5), and (7) the left-hand-side variable is the TB mortality per 1,000 people, and in columns (2), (4), and (6) the left-hand-side variable is the stacked causes of death from TB, cancer, influenza, pneumonia, accidents and suicides (including homicides from 1931), scarlet fever, diphtheria, and polio per 1,000 people. All regressions include city and year fixed effects, and addtionally columns (2), (4), and (6) include disease, disease-by-year, disease-by-city, and city-by-year fixed effects. TB dispensary $_{c, t}$ is an indicator variable equal to one after the introduction of a TB dispensary, and $\log \left(\right.$ Population $\left._{c, t-1}\right)$ is the lagged $\log$ population, where both variables are multiplied by an indicator equal to one if the disease on the left-hand side is TB. In columns (3) and (4) the initial log population (the log population of the first year the city is included in the sample) interacted with year fixed effects and multiplied by an indicator equal to one if the disease on the left-hand side is TB is included, and in column (7) a city-specific linear time trend is included. Robust standard errors clustered at the city level are in parentheses.

${ }^{*},{ }^{* *}$ and ${ }^{* * *}$ determine significance levels of 10 percent, 5 percent and 1 percent, respectively. 
Table A11: Effect of TB dispensaries controlling for commissioning of waterworks and the establishment of housing associations

\begin{tabular}{|c|c|c|c|c|}
\hline \multirow[t]{2}{*}{ Dep. variable: } & $\mathrm{TB}$ rate & Disease & $\mathrm{TB}$ rate & Disease \\
\hline & $(1)$ & $(2)$ & $(3)$ & $(4)$ \\
\hline TB dispensary ${ }_{c, t}$ & $\begin{array}{c}-0.262^{* * *} \\
(0.075)\end{array}$ & $\begin{array}{c}-0.214^{* * *} \\
(0.063)\end{array}$ & $\begin{array}{c}-0.260^{* * *} \\
(0.076)\end{array}$ & $\begin{array}{c}-0.213^{* * *} \\
(0.062)\end{array}$ \\
\hline Waterworks $_{c, t}$ & $\begin{array}{l}-0.101 \\
(0.091)\end{array}$ & $\begin{array}{r}-0.086 \\
(0.099)\end{array}$ & & \\
\hline Housing Association $_{c, t}$ & & & $\begin{array}{c}0.088 \\
(0.087)\end{array}$ & $\begin{array}{c}0.074 \\
(0.081)\end{array}$ \\
\hline Avg. TB rate & 1.306 & 1.034 & 1.306 & 1.034 \\
\hline City FE & Yes & Yes & Yes & Yes \\
\hline Year FE & Yes & Yes & Yes & Yes \\
\hline Disease FE & No & Yes & No & Yes \\
\hline City FE $\times$ Year FE & No & Yes & No & Yes \\
\hline Disease $\mathrm{FE} \times$ Year FE & No & Yes & No & Yes \\
\hline Disease FE $\times$ City FE & No & Yes & No & Yes \\
\hline Time period & 1890-1939 & 1901-1939 & 1890-1939 & 1901-1939 \\
\hline Observations & 3,981 & 25,320 & 3,981 & 25,320 \\
\hline R-squared & 0.535 & 0.697 & 0.534 & 0.697 \\
\hline Cities & 87 & 87 & 87 & 87 \\
\hline
\end{tabular}

Notes: The table reports least squares estimates. In columns (1) and (3) the left-hand-side variable is the TB mortality per 1,000 people, and in columns (2) and (4) the left-hand-side variable is the stacked causes of death from TB, cancer, influenza, pneumonia, accidents and suicides (including homicides from 1931), scarlet fever, diphtheria, and polio per 1,000 people. All regressions include city and year fixed effects, and addtionally columns (2) and (4) include disease, disease-by-year, disease-by-city, and city-by-year fixed effects. TB dispensary ${ }_{c, t}$ is an indicator variable equal to one after the introduction of a TB dispensary, Waterworks $c_{c, t}$ is an indicator equal to one after the introduction a waterwork, and Housing Association ${ }_{c, t}$ is an indicator equal to one after the introduction of a housing association, where all are multiplied by an indicator equal to one if the disease on the left-hand side is TB. Robust standard errors clustered at the city level are in parentheses.

${ }^{*},{ }^{* *}$ and ${ }^{* * *}$ determine significance levels of 10 percent, 5 percent and 1 percent, respectively. 
Table A12: Effect of main and branch TB dispensaries

\begin{tabular}{lccc}
\hline \hline Dep. variable: & TB rate & Disease \\
\cline { 2 - 2 } & $(1)$ & $(2)$ \\
\hline Main dispensary & & \\
& $-0.219^{* *}$ & & $-0.206^{* *}$ \\
Branch dispensary & $(0.091)$ & & $(0.086)$ \\
& $-0.289^{* *}$ & & $-0.204^{* *}$ \\
& $(0.114)$ & $(0.080)$ \\
\hline Avg. TB rate & 1.306 & 1.034 \\
\hline Joint significance of & & \\
main and branch dispensary & {$[0.006]$} & {$[0.007]$} \\
Test of equivalence between & & \\
main and branch dispensary & {$[0.616]$} & {$[0.979]$} \\
\hline City FE & Yes & Yes \\
Year FE & Yes & Yes \\
Disease FE & No & Yes \\
Disease FE $\times$ Year FE & No & Yes \\
Disease FE $\times$ City FE & No & Yes \\
City FE $\times$ Year FE & No & Yes \\
\hline Time period & $1890-1939$ & $1901-1939$ \\
Observations & 3,981 & 25,320 \\
R-squared & 0.53 & 0.70 \\
Cities & 87 & 87 \\
\hline
\end{tabular}

Notes: The table reports least squares estimates. In column (1) the left-hand-side variable is the TB mortality per 1,000 people, and in column (2) the left-hand-side variable is the stacked causes of death from TB, cancer, influenza, pneumonia, accidents and suicides (including homicides from 1931), scarlet fever, diphtheria, and polio per 1,000 people. All regressions include city and year fixed effects, and addtionally column (2) includes disease, disease-by-year, disease-by-city, and city-by-year fixed effects. Main dispensary ${ }_{c, t}$ is an indicator variable equal to one after the introduction of a main TB dispensary, and Branch dispensary ${ }_{c, t}$ is an indicator variable equal to one after the introduction of a branch TB dispensary, where both indicators are multiplied by an indicator equal to one if the disease on the left-hand side is TB. Robust standard errors clustered at the city level are in parentheses, and p-values in square brackets.

${ }^{*},{ }^{* *}$ and ${ }^{* * *}$ determine significance levels of 10 percent, 5 percent and 1 percent, respectively. 
Table A13: Effect of TB dispensaries in a balanced and extended panel

\begin{tabular}{|c|c|c|c|c|}
\hline \multirow[t]{3}{*}{ Dep. variable: } & TB rate & Disease & TB rate & Disease \\
\hline & \multicolumn{2}{|c|}{ Balanced panel } & \multicolumn{2}{|c|}{ Panel extended to 1946} \\
\hline & $(1)$ & $(2)$ & $(3)$ & $(4)$ \\
\hline TB dispensary dit $_{t}$ & $\begin{array}{c}-0.274^{* * *} \\
(0.084)\end{array}$ & $\begin{array}{c}-0.232^{* * *} \\
(0.071)\end{array}$ & $\begin{array}{c}-0.219^{* * *} \\
(0.058)\end{array}$ & $\begin{array}{c}-0.160^{* * *} \\
(0.049)\end{array}$ \\
\hline Avg. TB rate & 1.340 & 1.052 & 1.176 & 0.920 \\
\hline City FE & Yes & Yes & Yes & Yes \\
\hline Year FE & Yes & Yes & Yes & Yes \\
\hline Disease FE & No & Yes & No & Yes \\
\hline Disease $\mathrm{FE} \times$ Year $\mathrm{FE}$ & No & Yes & No & Yes \\
\hline Disease FE $\times$ City FE & No & Yes & No & Yes \\
\hline City $\mathrm{FE} \times$ Year FE & No & Yes & No & Yes \\
\hline Time period & 1890-1939 & 1901-1939 & 1890-1946 & $1901-1946$ \\
\hline Observations & 3,700 & 23,088 & 4,590 & 30,192 \\
\hline R-squared & 0.54 & 0.71 & 0.57 & 0.70 \\
\hline Cities & 74 & 74 & 87 & 87 \\
\hline
\end{tabular}

Notes: The table reports least squares estimates. In columns (1) and (3) the left-hand-side variable is the TB mortality per 1,000 people, and in columns (2) and (4) the left-hand-side variable is the stacked causes of death from TB, cancer, influenza, pneumonia, accidents and suicides (including homicides from 1931), scarlet fever, diphtheria, and polio per 1,000 people. All regressions include city and year fixed effects, and addtionally column (2), and (4) includes disease, disease-by-year, disease-by-city, and city-by-year fixed effects. TB dispensary ${ }_{c, t}$ is an indicator variable equal to one after the introduction of a TB dispensary multiplied by an indicator equal to one if the disease on the left-hand-side is TB. In columns (1) and (2) the sample are the 74 cities that constitutes a balanced panel, and in columns (3) and (4) the sample is extended to also include the years 1940 to 1946. Robust standard errors clustered at the city level are in parentheses.

${ }^{*},{ }^{* *}$ and ${ }^{* * *}$ determine significance levels of 10 percent, 5 percent and 1 percent, respectively. 